\title{
EVIDENCE-BASED DEVELOPMENT AND INITIAL VALIDATION OF THE PAIN ASSESSMENT CHECKLIST FOR SENIORS WITH LIMITED ABILITY TO COMMUNICATE-II (PACSLAC-II)
}

\author{
A Thesis \\ Submitted to the Faculty of Graduate Studies and Research \\ In Partial Fulfillment of the Requirements \\ For the Degree of \\ Doctor of Philosophy \\ in \\ Clinical Psychology \\ University of Regina \\ By \\ Sarah Lok Chuen Chan \\ Regina, Saskatchewan
}

May 2013

Copyright 2013: S. L. C. Chan 


\section{UNIVERSITY OF REGINA}

\section{FACULTY OF GRADUATE STUDIES AND RESEARCH SUPERVISORY AND EXAMINING COMMITTEE}

Sarah Lok Chuen Chan, candidate for the degree of Doctor of Philosophy in Clinical Psychology, has presented a thesis titled, Evidence-Based Development and Initial Validation of the Pain Assessment Checklist for Seniors with Limited Ability to Communicate-II (PACSLAC-II), in an oral examination held on June 4, 2013. The following committee members have found the thesis acceptable in form and content, and that the candidate demonstrated satisfactory knowledge of the subject material.

External Examiner:

Supervisor:

Committee Member:

Committee Member:

Committee Member:

Chair of Defense:

*via Video Conference
${ }^{\star}$ Dr. Victor Molinari, University of South Florida

Dr. Thomas Hadistavropoulos, Department of Psychology

Dr. Katherine Robinson, Department of Psychology

Dr. Kristi Wright, Department of Psychology

Dr. Darren Candow, Faculty of Kinesiology \& Health Studies

Dr. Martin Argerami, Department of Mathematics \& Statistics 


\begin{abstract}
Pain is undertreated in long-term care (LTC) facilities, in part because of difficulties in evaluating pain among seniors with dementia and limited ability to communicate (Wynne, Ling, \& Remsburg, 2000). One of the leading assessment tools for this population is the Pain Assessment Checklist for Seniors with Limited Ability to Communicate (PACSLAC; Fuchs-Lacelle \& T. Hadjistavropoulos, 2004). Although the PACSLAC has strong psychometric properties and is frequently preferred by LTC staff for its clinical utility (Lints-Martindale, T. Hadjistavropoulos, Lix \& Thorpe, 2012; Zwakhalen, Hammers \& Berger, 2006), it consists of 60 items that may be considered to be too many for use at some busy settings. Moreover, like most tools of its kind, it contains items that overlap with conditions that may be unrelated to pain (e.g., delirium). The goal of this study was to develop the PACSLAC-II, a shorter tool that would improve on the PACSLAC. Revisions to the PACSLAC resulted in the PACSLAC-II which is of shorter length, contains items that are more pain-specific and continues to cover all pain assessment domains that are deemed to be important by the American Geriatrics Society (AGS) Panel on Persistent Pain in Older Persons (2002). Psychometric properties and clinical utility of the 31-item PACSLAC-II were then examined through two studies. In the first study, the PACSLAC-II was used to assess pain based on video footage of LTC residents with dementia undergoing painful procedures as part of routine care. Its ability to discriminate pain from non-pain related states was compared to that of pre-existing tools using archival data. The PACSLAC-II demonstrated satisfactory reliability and excellent validity. As well, it discriminated across pain-related situations and baseline while accounting for more variance than six other tools that are commonly
\end{abstract}


considered in the literature, including the original PACSLAC. Moreover, the PACSLACII accounted for unique variance with respect to ability to discriminate across pain-related situations and baseline, even after controlling for all other measures combined. A second study involved the use of the PACSLAC and PACSLAC-II in LTC facilities by front line nurses and care aides in order to solicit feedback from health care providers. Qualitative analysis of this feedback was conducted. Many LTC nurses and care aides preferred the PACSLAC-II due to its length and condensed nature, which may facilitate documentation and greater efficiency in pain assessment and management. However, a smaller number of nurses and care aides reported preference for the original PACSLAC. Despite the preference for the PACSLAC expressed by some staff members, the psychometric examination of the tools indicated that the shorter version had better discriminative properties. Feedback interview data also revealed that both tools were considered to be clinically useful by the LTC staff. Overall, the findings of this investigation indicate that the empirical and theoretically-driven revisions to the PACSLAC led to improved ability to differentiate between pain and non-pain states, while retaining its clinical utility for front line staff. Future studies of the PACSLAC-II may focus on examining its psychometric properties in LTC settings and its role in pain management decisionmaking. 


\section{ACKNOWLEDGEMENTS}

I am greatly indebted to many individuals without whose guidance and support the completion of this project would not have been possible.

First, I would like to express my deep gratitude to my dissertation supervisor, Dr. Thomas Hadjistavropoulos, for his indispensible expertise through the research process. His contagious energy, drive for excellence, and dedication to the field of pain assessment for persons with dementia were major forces behind the completion of this work. Secondly, I would like to thank my dissertation committee members, Drs. Katherine Robinson, Kristi Wright and Darren Candow, whose expertise and meticulousness improved the quality of this project and document. Thirdly, my sincere gratitude is extended to the older adults, nurses, and care aides who participated in this study, as well as directors of care who facilitated this research. I would also like to thank Dr. Amanda Lints-Martindale, whose work and efforts came before this project. I am also grateful to Dr. Jaime Williams, Kerry Spice, Eleni Gardikiotis and Kirstie Walker for their help in completing this project. Finally, I would like to acknowledge financial support from the Alzheimer Society of Canada, Saskatchewan Health Research Foundation and the Faculty of Graduate Studies and Research, University of Regina. 


\section{TABLE OF CONTENTS}

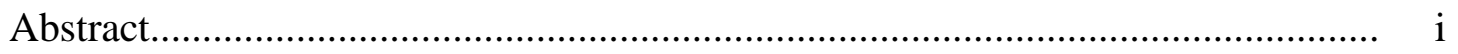

Acknowledgements.................................................................................. iii

Table of Contents.............................................................................. iv

List of Tables....................................................................................... viii

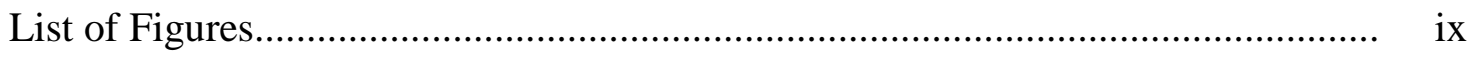

List of Appendices.....................................................................................

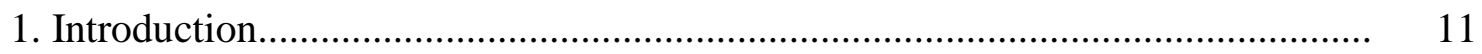

1.1 Overview................................................................................ 11

1.2 Pain as a Subjective Phenomenon...................................................... 13

1.3 Theoretical Issues related to Pain........................................................ 14

1.3.1 Unidimensional model of pain............................................. 14

1.3.2 Gate control theory of pain.................................................. 15

1.3.3 Neuromatrix model of pain.................................................. 17

1.3.4 Biopsychosocial model of pain............................................. 18

1.3.5 Communications model of pain.......................................... 18

1.4 Pain in Older Adults with Dementia................................................... 20

1.4.1 Definition of dementia......................................................... 20

1.4.2 Pain prevalence among older adults with dementia.................. 22

1.4.3 Self-report in older adults with dementia................................ 23

1.4.4 Pain processing and perception in older adults with dementia.... $\quad 24$

1.4.5 Underassessment and undermanagement of pain..................... 26

1.4.6 Consequences of unmanaged pain........................................... 29 
1.5 Observational Pain Assessment in Older Adults with Dementia............. 32

1.5.1 Development of observational pain assessment tools................. 34

1.5.2 Comparisons and reviews of observational pain tools............... 35

1.5.3 Development of the PACSLAC................................................. 42

1.5.4 Further research on the PACSLAC......................................... 44

1.6 Review of Research on Pain Behaviours............................................ 49

1.6.1 Facial expressions........................................................... 49

1.6.2 Verbalizations and vocalizations.......................................... 56

1.6.3 Body movements.......................................................... 57

1.6.4 Mental status changes and overlap with delirium................... 64

1.6.5 Changes in interpersonal interactions and activity patterns........ 67

1.6.6 Physiological indicators................................................... 68

1.7 Revisions of the PACSLAC .............................................................. 70

2. Method and Results............................................................................... 71

2.1 Study 1: Quantitative Comparison of the PACSLAC and PACSLAC-II... 71

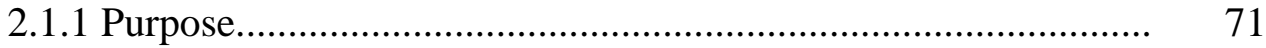

2.1.2 Resident videos................................................................. 71

2.1.3 Description of sample.................................... 77

2.1.4 Coding of videos............................................................ $\quad 78$

2.1.5 Measures....................................................................... $\quad 79$

2.1.5.1 Cornell Scale for Depression in Dementia...................... 79

2.1.5.2 Assessment of Discomfort in Dementia........................ 79

2.1.5.3 Checklist of Nonverbal Pain Indicators.......................... 80 
2.1.5.4 Pain Assessment for the Dementing Elderly scale........... 80

2.1.5.5 Pain Assessment in Advanced Dementia Scale............... 81

2.1.5.6 Non-Communicative Patient's Pain Assessment

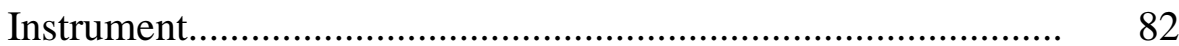

2.1.6 Hypothesis.................................................................. 83

2.1.7 Analyses and Results..................................................... 83

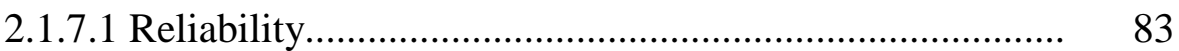

2.1.7.2 Validity ................................................................. 84

2.1.7.3 Convergent validity................................................... 84

2.1.7.4 Discriminant validity............................................. 84

2.1.7.5 Ability to discriminate between pain and baseline

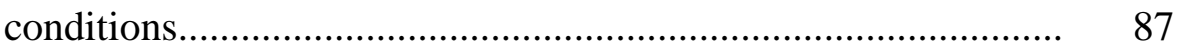

2.1.7.6 Comparisons with other observational pain tools........... 90

2.1.3.6.1 Effect size...................................................... 90

2.1.3.6.2 Controlling for contribution from other pain

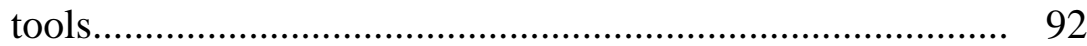

2.1.7.7 Exploration of individual items and subscales................ 93

2.1.3.7.1 Frequency of endorsement................................ 93

2.1.3.7.2 Item-total correlation...................................... 96

2.1.3.7.3 Subscale ability to differentiate across conditions. 96

2.2 Study 2: Feedback from LTC Nurses and Special Care Aides............... 99

2.2.1 Purpose........................................................................... 99

2.2.2 Participants............................................ 102 
2.2.3 Procedure............................................................................. 102

2.2.3.1 PACSLAC instructions........................................................ 103

2.2.3.2 Staff Interviews............................................................ 104

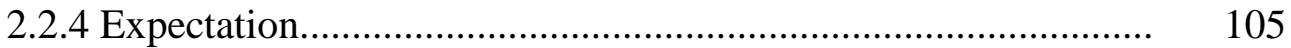

2.2.4 Analysis of ratings and interview data......................................... 105

2.2.6 Results of the comparison based on tool used.............................. 109

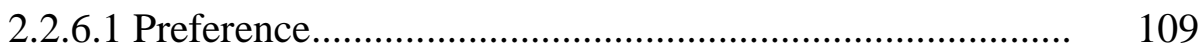

2.2.6.2 Facilitators and barriers.................................................... 111

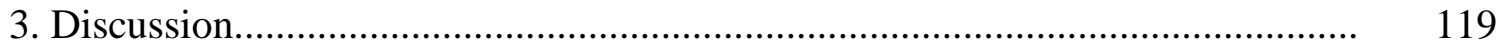

3.1 Revisions, Validation and Implications...................................................... 120

3.2 Front Line Use....................................................................................... 123

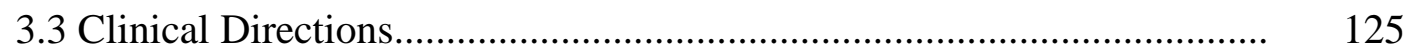

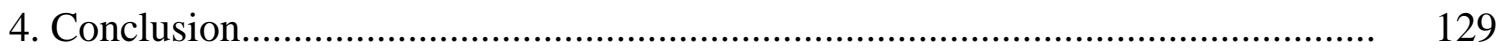

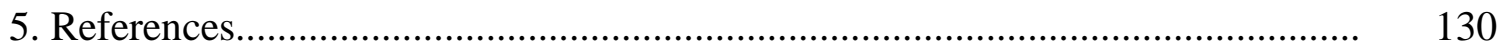




\section{LIST OF TABLES}

Table 1.1 Facial expressions associated with pain based on FACS.................. 51

Table 1.2 Verbalizations and vocalizations associated with pain.................... 58

Table 1.3 Body movements associated with pain........................................ 61

Table 2.1. Internal consistency of PACSLAC-II scores............................... 85

Table 2.2 Correlations between PACSLAC-II and other measures for the swabbing, influenza vaccination, and movement-exacerbated pain

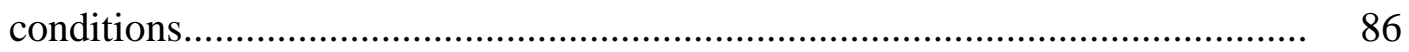

Table 2.3 Means and standard deviations (SDs) of PACSLAC-II scores.......... 88

Table 2.4 Paired comparisons of PACSLAC-II ANCOVA results with

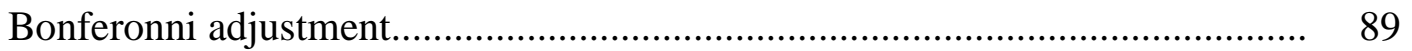

Table 2.5 Effect sizes (partial $\eta^{2}$ ) from ANOVAs comparing pain condition

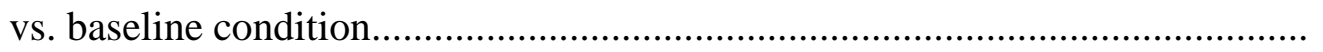

Table 2.6 Percentage frequency of PACSLAC-II items coded during each

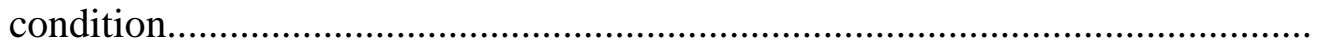

Table 2.7 Item-total correlations of PACSLAC-II scores.

Table 2.8 Paired comparisons of Facial Expression Subscale ANCOVA results with Bonferonni adjustment........................................

Table 2.9 Paired comparisons of Body Movement Subscale ANCOVA results with Bonferonni adjustment........................................... 101

Table 2.10 Responses to questions concerning tool clinical utility............ 106

Table 2.11 Responses to questions concerning tool clinical utility............ 107 


\section{LIST OF FIGURES}

Figure 1.1 PACSLAC items arranged by AGS domains................................. 72

Figure 1.2 Elimination of PACSLAC items overlapping with delirium............ 73

Figure 1.3 Revision of PACSLAC items..................................................... 75

Figure 2.1 Similarities and differences on the facilitators category.................. 112

Figure 2.2 Similarities on the barriers category.......................................... 117 


\section{LIST OF APPENDICES}

Appendix A. PACSLAC-II .......................................... 160

Appendix B. Instructions for using pain checklists ............................ 161

Appendix C. PACSLAC-II pain graph..................................... 164

Appendix D. Staff participant information form........................... 165

Appendix E. Staff feedback ratings form.................................. 166

Appendix F. Qualitative analysis code book.............................. 167

Appendix G. University of Regina Research Ethics Board approval............ 169 


\section{Introduction}

\subsection{Overview}

Pain is a subjective internal experience that requires communication between the individual in pain and an observer in order for assessment and treatment to occur. The frequent use of verbal self-report as the gold standard for pain assessment is a reflection of the importance of the individual's subjective experience of pain over outward signs of injury (Craig, 2009). However, use of self-report requires ability to communicate verbally and this poses a unique challenge for managing pain in persons with cognitive impairment and limited ability to communicate. At present, about $45 \%$ of older adults with dementia and difficulty with verbal communication reside in LTC facilities (Alzheimer Society of Canada, 2010). Based on numerous studies in the past decade, it is known that many LTC residents with dementia are living with underassessed and undertreated pain (Feldt, Ryden, \& Miles, 1998; Ferrell, Ferrell, \& Rivera, 1995; Miller, Nelson, \& Mezey, 2000; Morrison \& Siu, 2000). Undermanagement of pain in older adults with dementia has been found in various settings, including acute hospital care, palliative care, and LTC facilities (e.g., Afzal, Buhagiar, Flood, \& Cosgrave, 2010; Herr

et al., 2004; Reynolds, Hanson, DeVellis, Henderson, \& Steinhauser, 2008). A clear finding across these studies is that older adults with dementia often receive less scheduled and p.r.n. (pro re nata) medication compared to those without dementia despite comparable painful medical diagnoses or injury (Kaasalainen et al, 1998). This disparity in pain management between older adults with and without dementia is likely due in part to the residents' difficulty in communicating pain to care staff, as well as the challenge in accurately identifying pain in these residents (Kaasalainen et al., 2007). 
Because one cannot rely solely on verbal self-report to adequately manage pain in older adults with dementia, it has been recommended that nonverbal pain behaviours be assessed (AGS Panel on Persistent Pain in Older Persons, 2002). Indeed, nurses report attending to nonverbal cues of pain in their patients, though non-systematically (Mentes, Teer, \& Cadogan, 2004). The past decades saw the development of numerous observational pain assessment tools for more systematic assessment of pain behaviours in order to address this problem (Herr, Bjoro, \& Decker, 2006). Among the tools developed, the PACSLAC is the tool that comprehensively covers all six behavioural domains of nonverbal behaviours deemed to be important for the pain assessment of older adults with dementia (AGS Panel on Persistent Pain in Older Persons, 2002; Fuchs-Lacelle \& Hadjistavropoulos, 2004). As well, compared to other tools of its kind, the PACSLAC has demonstrated comparably favourable reliability and ability to discriminate between painful and non-painful states (Lints-Martindale et al., 2012; Zwakhalen et al., 2006b). While the existing literature on the PACSLAC has demonstrated a number of positive qualities of the instrument, as with many observational pain tools, the PACSLAC contains items that are not pain-specific and overlap with other conditions such as delirium. Moreover, the PACSLAC, with 60 items, is considerably longer than other tools of its kind and, thus, may be more cumbersome to use in some busy clinical settings. The aim of this study was to address these issues and in so doing, improve the psychometric properties and clinical utility of this observational pain assessment tool.

This investigation was conducted in 3 parts: (1) revision of the PACSLAC based on theoretically and empirically driven modification of the items; (2) examination of the reliability and validity of the resulting PACSLAC-II using archival footage of LTC 
residents undergoing painful procedures; and (3) use of the tool in LTC facilities with nurses and care aides who then provided qualitative feedback about the PACSLAC and the PACSLAC-II.

\subsection{Pain as a Subjective Phenomenon}

Pain is a complex and subjective experience that comprises of nociceptive, perceptual, cognitive, emotional and social elements (Turk, 2001). According to the International Association for the Study of Pain (IASP), pain is defined as "an unpleasant sensory and emotional experience associated with actual or potential tissue damage” (Merskey \& Bogduk, 1994). This definition of pain recognizes that while pain serves the essential sensory purpose of signalling tissue damage, pain is also defined in part by an individual's emotional experience regardless of physical signs of injury (Turk, 2001). A widely cited definition also emphasizes the subjective nature of pain, where pain is "whatever the experiencing person says it is and exists whenever he or she says it does" (McCaffery \& Beebe, 1989). Therefore, though pain is a universal human phenomenon, the experience of pain is subjective. For example, individuals who have sustained similar degrees of tissue damage have reported widely different pain experiences, including pain severity, beliefs about pain, as well as emotional and behavioural responses (Turk, 2001). Thus, the method considered as the "gold standard" of detecting and understanding pain in another is self-report (Smith, 2005). While eliciting self-report acknowledges and respects the individual's subjective experience of pain, many individuals, such as infants or individuals with cognitive impairment, lack the ability to communicate this information. As such, IASP explicitly recognizes that any limitation in ability to express pain does not mean the individual does not experience pain (Merskey \& Bogduk, 1994). 
Instead, when individuals are unable to communicate pain, it becomes the observer or caregiver's responsibility to identify pain. The focus of this research is on the subsample of older adults who, as a result of dementia, experience significant limitations in their ability to communicate their pain experience.

Our current understanding of pain as a subjective multidimensional experience (i.e., sensory, perceptual, cognitive, psychosocial) is based on many years of research and transformations in the understanding of pain. Early understandings were focused on pain as a sensory and physiological process and can be traced back to Descartes in the $15^{\text {th }}$ century (Turk, 2001). Since that time, important theoretical and empirical developments have led up to and constitute the conceptual foundation of current psychological research on pain, including the basis of the present study. These include the gate control theory, the neuromatrix model of pain, as well as the biopsychosocial and the communications model of pain.

\subsection{Theoretical Issues related to Pain}

1.3.1 Unidimensional model of pain. Though conceptualizations of pain date back to the ancient Greeks, Descartes was likely the first to formally conceptualize pain as a unidimensional sensory experience (Turk, 2001). Within a mechanistic view of the human body, Descartes described pain as a linear sequence of events, where contact of noxious stimulus to a pain receptor leads to the transmission of a pain signal from the receptor to the brain, resulting in the recognition of pain. Descartes' conceptualization highlighted the existence of a pathway from the site of stimulation to the brain, likening the transmission to pulling a string that rings a bell, signalling pain. This unidimensional model of pain assumed a one-to-one relationship between the amount of pain stimulus 
and the amount of pain experienced, a notion which indeed accounted for general observations about injury and pain (i.e., when one's injury is extensive, one would expect more intense pain). Advances in anatomical knowledge in the 1800s further solidified the unidimensional model of pain, where anatomists found evidence that specific pathways, receptors and locations on the spinal cord were involved in the transmission of pain signals (Cope, 2009). A number of anatomists at this time, including Moritz Schiff, Max von Frey, amongst others, based their investigations on a specificity model of pain in tracing the physical basis of pain signals (Cope, 2009). However, a conceptualization of pain where amount of pain is proportional to tissue damage does not account for many individual experiences, such as those with phantom limb pain and chronic pain. As such, many individuals who did not exhibit signs of injury but were experiencing pain were erroneously believed to be psychologically disordered (Hadjistavropoulos \& Craig, 2003d; Melzack \& Katz, 2004).

1.3.2 Gate control theory of pain. Melzack and Wall (1965) published a seminal paper which construed pain as a complex phenomenon where pain is not solely explained by tissue damage. The gate control theory of pain drew attention to dynamic processes in the central nervous system that modulated pain rather than acting as a passive pathway (Melzack \& Wall, 1965; Melzack \& Katz, 2004). In particular, it was posited that a mechanism in the spinal cord facilitated the transmission and gating of nociceptive signals. The gating mechanism was thought to be located in the dorsal horns of the spinal cord where signals ascending from receptors meet descending signals from the cortex. Specifically, two types of fibers carry ascending signals: small $A$ myelinated fibers that transmit nociceptive (i.e., pain) information and large $C$ fibers that carry non-nociceptive 
(e.g., pressure) information. The balance of information from the two types fibers affects the nociceptive information that is sent to the brain. For example, more activity along the $C$ fibers will have an inhibitory effect on the $A$ fibers, the phenomenon at work when rubbing a sore area leads to lessening of pain experienced. Additionally, information is thought to descend from the cortex to the dorsal horn, also influencing the resultant signal sent to the brain. For example, when an individual focuses attention on or anticipates pain, this can facilitate the transmission of nociceptive information to the brain. Thus, the gating mechanism or pain gate is constituted by the mutual inhibition and summation of ascending and descending signals. This conceptualization of the central nervous system sheds light on the role of cortical input, where the experience of pain as absent or present is partly controlled by descending signals (Melzack \& Katz, 2004). For instance, cognitive and affective states, such as attending to pain sensations at tissue damage, can lead to greater likelihood of opening the gate, and the amplification of the pain experience. In this way, the gate control theory was one of the first theories to emphasize both physiological and psychological aspects of pain perception and pain experience. Additionally, the theory describes pain experience as the result of dynamic processes in the brain and receptor, which may help account for the lack of one-to-one relationship between injury and pain, as well as the subjective experiences of individuals in pain. Finally, the theory provided justification for the study of psychological mechanisms that may be involved in the pain experience (Gagliese \& Katz, 2000). The gate control theory has received a great deal of empirical support (Sufka \& Price, 2002) and studies continue to be conducted based on the notion that pain experience is a result of the summation and inhibition of ascending and descending information (e.g., Julien \& Marchand, 2006; 
Julien, Goffaux, Arsenault, \& Marchand, 2005; Sufka \& Price, 2002). With regard to this study, the gate control theory helps us understand the challenges of assessing pain in people with limited ability to communicate especially because the theory highlights the physiological mechanisms that contribute to the subjectivity of pain (Turk, 1996).

1.3.3 Neuromatrix model of pain. The neuromatrix model, proposed by Melzack (Melzack \& Katz, 2004), complements the gate control theory of pain and helps explain phenomena such as phantom limb pain (Hill, 1999). The model focuses on the fundamental role that the brain plays in the experience of pain. The neuromatrix refers to the networks of interconnecting neurons in the brain that are responsible for processing information giving rise to conscious experience of pain. A unified awareness of the body and the specific qualities of the pain experience (e.g, sensations, emotions, cognitions, motor activity) are thought to be a product of this coordinated neural activity. Based on this model, when a nociceptor is stimulated, and resultant signals ascend through the spinal cord and reach the brain, networks are activated which then produce the conscious experience of pain. Specifically, cortical activity alone is responsible for producing the conscious experience of pain, such that while ascending signals may activate or modify it, the conscious experience of the body is located in cortical networks. Thus, it follows that for individuals with severed spinal cords or limbs, the lack of ascending pain signals does not necessarily eliminate pain experience. Taken together, the gate control theory and neuromatrix model have helped explain how individuals with similar pain etiologies experience pain in diverse ways. Specifically for older persons with dementia, the gate control theory and neuromatrix model emphasize the importance of an individualized approach to assessing and treating pain. 
1.3.4 Biopsychosocial model of pain. Consistent with the gate control theory and neuromatrix model, is the biopsychosocial model of pain, which is the most predominant pain model today (Asmundson \& Wright, 2004; T. Hadjistavropoulos, Craig, Duck, et al., 2011; Turk \& Flor, 1999). As evident from its name, the biopsychosocial model emphasizes the interaction among biological, psychological and social factors in an individual's pain experience. For example, persons with persistent pain often suffer psychological and social consequences, such as anxiety, disability and job loss, but these factors also influence the individual's perception of pain and the course of his or her rehabilitation. Further, most pain clinicians recognize the importance of psychological and social variables as well as biological ones in pain treatment and thus are evaluated as part of a comprehensive pain assessment (T. Hadjistavropoulos, S. Gibson, \& Fine, 2011). Research within the biopsychosocial framework has led to the development of such knowledge as the operant model of pain (i.e., where an individual's behavioural responses to pain carry social consequences that reinforce the behaviours; Fordyce, 1976), or the biobehavioural model (i.e., which recognizes the interaction among genetic predisposition and cognitive, behavioural, social and physiological responses; Turk, Meichenbaum, \& Genest, 1983). Support for the biopsychosocial model of pain is seen not only in the work on pain rehabilitation, where treatments dealing with pain-related emotional, behavioural and occupational concerns lead to improved outcomes, but also in a variety of other social and cultural contexts (T. Hadjistavropoulos, Craig, Duck, et al., 2011; Morley, Williams, \& Hussain, 2008; Turk et al., 2008).

1.3.5 Communications model of pain. As an extension of the biospsychosocial model, the communications model of pain (T. Hadjistavropoulos \& Craig, 2002; T. 
Hadjistavropoulos, Craig, \& Fuchs-Lacelle, 2004; T. Hadjistavropoulos, Craig, et al., 2011; Prkachin \& Craig, 1995) details the relationship between an individual's pain experience and observers' understanding of that pain and focuses on the social context in which this interaction occurs. While the gate control theory, neuromatrix model and biopsychosocial model form the foundations of pain research today, the communications model of pain may be most pertinent to the present study. Specifically, the communications model describes the chain of events that takes place when pain is experienced in an interpersonal context: (1) the individual experiences pain which can be affected by a variety of sociocultural parameters (Portenoy, Ugarte, Fuller, \& Haas, 2004); (2) the individual encodes pain into verbal, nonverbal and physiological displays; (3) an observer decodes the displays as meaning pain, and decides whether or not to respond by providing care or emotional support. Further, each stage in pain communication is complicated by the influence of intrapersonal and contextual factors. For instance, when an older adult with severe dementia experiences pain, his or her pain expression (i.e., encoding) may be limited by motor and cognitive abilities due to brain pathology. Contextual factors, such as social and cultural rules regarding pain expression, also influence the encoding of pain (Hobara, 2005). In terms of the identification (i.e., decoding) of pain, the observer is influenced by his or her attention and attitude, as well as his or her relationship to the individual in pain (Kappesser \& de C. Williams, 2002; Pillai Riddell \& Craig, 2007). While the events detailed in the communications model are complex, a basic assumption of this perspective is that the ability to communicate pain emerged out of human evolution due to the survival and reproductive benefits of this ability. Thus, it is assumed that most individuals engage in pain communication and that 
many of the actions involved (i.e., pain-related facial expressions) have universal elements and occur automatically (T. Hadjistavropoulos, Craig, et al., 2011).

Applied to the context of LTC, when caring for an older adult with dementia, the caregiver must accurately decode all available pain expressions in order to proceed with appropriate care. As suggested by the model, this process is complicated by extraneous factors. Encoding of pain may be limited by the older adult's ability to verbalize pain, while nonverbal pain indicators are also known to be idiosyncratic (Foley \& McCutcheon, 2004). At the same time, decoding of pain is affected by the caregiver's attention (e.g., whether he or she observed the pain behaviour; de Rond et al., 2000), beliefs (e.g., whether the behaviour is consistent with what the caregiver perceives as indicating pain; Weiner, Peterson, \& Keefe, 1999), clarity of the pain expression (e.g., nonverbal messages are more difficult to decode than verbal ones; Hale \& Hadjistavropoulos, 1997), relationship to the older adult (Burgener \& Twigg, 2002) and social context (e.g., dementia unit; Alexander et al., 2005). It was the aim of this study to develop a tool that would facilitate the communication of pain between an older person with dementia and his or her caregiver. Specifically, an observational pain assessment tool may facilitate the decoding of behaviour, by influencing how the staff member attends to the resident or how the staff member interprets the observed behaviours.

\subsection{Pain in Older Adults with Dementia}

1.4.1 Definition of dementia. The term dementia describes a syndrome of decline in cognitive and behavioural functions and covers well over 50 conditions with differing causes and prognoses (Zillmer, Spiers, \& Culbertson, 2008). Many dementias are progressive and result from an underlying disease process, while some are static, treatable 
and may be caused by injury or exposure to toxicity. However, a major common feature of the dementias is the loss of multiple areas of cognitive functions, such as memory, language, or problem solving.

According to the Diagnostic and Statistical Manual of Mental Disorders-IV-TR (DSM-IV-TR; American Psychiatric Association, 2000), dementia is characterized by the development of (1) memory impairment, with at least one of: aphasia, apraxia, agnosia, or disturbance in executive functioning and (2) a decline from previous level of functioning. Aphasia, in particular, describes the decline in language ability, where the individual will experience difficulty with expression of language (e.g., naming objects, forming sentences) and comprehension of written and spoken language. In individuals with severe dementia, language function is often greatly impaired, with the individual becoming mute, non-communicative, echolalic (i.e., echoing what is heard), or palilalic (i.e., repeating words or sounds). Aside from decline in verbal ability, dementia can also involve agnosia, the loss of ability to recognize or attach meaning to objects, people, sounds, smells and shapes. With agnosia, individuals may not recognize family members, their own reflection in the mirror, or simple objects such as a pencil or hair brush. In addition, dementia can also involve the loss of ability to execute motor activities despite intact sensorimotor abilities and comprehension of the task, or otherwise known as apraxia. Individuals with apraxia may have difficulty carrying out precise limb movement, facial movements, motor acts (e.g., pick up this pencil), and might also have difficulty executing motor movement necessary for speech. Finally, loss of executive function is also common in dementia. Individuals with impairment in executive function tend to show difficulty with planning, initiating, sequencing, monitoring, and stopping 
complex behaviour, thus leading to interference with abstract reasoning, problem solving, and coping with novel situations. All these types of decline limit a person's ability to effectively and reliably communicate pain.

Dementia is also categorized based on etiology (American Psychiatric Association, 2000). Among the different types of dementia, Alzheimer's disease is the most common, where 50 to $60 \%$ of all dementia cases are due to Alzheimer's disease (Blennow, de Leon, \& Zetterberg, 2006). Alzheimer’s disease is also more prevalent in older adults over the age of 65 years, with 1\% prevalence in those between 60 to 64 years and increasing exponentially with age. Other types of dementia have also been investigated, such as Lewy-body disease, Creutzfeldt-Jakob disease, vascular dementia and frontotemporal dementia (Scherder, Sergeant, \& Swaab, 2003). It is estimated that in LTC facilities, at least half of the residents are living with dementia (Ferrell, Ferrell, \& Rivera, 1995; Parmalee, 1996). According to a recent report commissioned by the Alzheimer Society of Canada (2010), currently about 480,000 Canadians over age 65 are living with dementia. Of these individuals, about $45 \%$ are residents in LTC facilities. With the projected boom of the older cohort in the coming years, it is estimated that there will be at least 1.1 million Canadians over age 65 with dementia by the year 2038, with approximately 440,000 residing in LTC facilities. Thus, LTC staff will inevitably face a surge in demand for care of residents with dementia, along with a need to meet the challenges therein, such as adequate pain management.

1.4.2 Pain prevalence among older adults with dementia. As cognitive decline in dementia likely impedes older adults' ability to report pain (Oosterman, de Vries, Dijkerman, de Haan, \& Scherder, 2008), prevalence of pain in this population has largely 
been determined by examining the prevalence of painful conditions. Specifically, by examining chart diagnoses that are likely to cause pain, it was found that $48 \%$ of residents with moderate to severe dementia lived with painful diseases (Reynolds et al., 2008). This prevalence was comparable to the pain prevalence in cognitively intact residents. A large study of LTC residents in Canada also confirmed the prevalence of pain in this population at 50\%, with residents with cognitive impairment and those who are cognitively intact being equally likely to be living with painful health conditions (Proctor \& Hirdes, 2001). Ferrell, Ferrell and Rivera (1995) have argued that the prevalence of pain in LTC residents with or without dementia is likely to be as high as $70 \%$, the known prevalence of arthritis in this population. In addition, arthritis was found to be the chief reason for a pain complaint in this group, followed by old fractures $(13 \%)$, neuropathies (10\%), malignancies (4\%), and muscle spasm (1\%; B. A. Ferrell et al., 1995). Other conditions related to pain reports from LTC residents also include osteoporosis, pressure ulcers and infections, amongst others (Proctor \& Hirdes, 2001). For residents with dementia, pain was additionally related to verbally abusive behaviour, depression, unsteady gait and recent falls.

1.4.3 Self-report in older adults with dementia. In order to self-report pain, cognitive processing must take place. An individual must be able to perceive and understand questions about pain and to respond about the pain experienced. For older adults with dementia, this chain of cognitive and motor processes is hampered by neurodegeneration (Zillmer et al., 2008). Indeed, researchers have found that older adults with dementia are less able to locate their pain compared to cognitively intact older adults (M. Schuler, Njoo, Hestermann, Oster, \& Hauer, 2004). While a selection of self-report 
pain tools are available, capturing self-report of pain from older adults with dementia is often unfeasible, especially in cases of moderate to severe dementia. When examining five simple self-report pain ratings scales, about $17 \%$ of LTC residents were unable to respond verbally about their pain (Ferrell, Ferrell, \& Rivera, 1995). In particular, residents with a Modified Mini-mental Status Exam (Folstein, Folstein, \& McHugh, 1975) score of 15 or less were significantly less able to respond to questions about pain intensity and pain locations (Wynne et al., , 2000). Thus, while many residents with mild cognitive impairment are able to self-report pain, self-report instruments may not be useful for those who are more cognitively impaired. Also, when older persons with mild to moderate dementia enter acute care settings, they may encounter difficulties with selfreports of pain. While hospitalized, older persons with mild to moderate dementia were found to have difficulty describing qualitative features of their pain, reporting side effects, as well as identifying changes in pain intensity (Kelley, Siegler, \& Reid, 2008).

It is apparent that cognitive impairment due to dementia presents a major barrier to pain assessment and management (Ferrell et al., 1995). Due to difficulties facing older adults with dementia to verbally self-report pain, greater responsibility is then placed on caregivers to identify pain in their care-recipients. Compounded by the subjective nature of pain, and insufficient education in pain management for LTC nurses and care aides (Allcock, McGarry, \& Elkan, 2002), adequate pain management is a great challenge. However, recent findings regarding the pain processing and perception of older adults with dementia may begin to elucidate their subjective pain experience.

1.4.4 Pain processing and perception in older adults with dementia. While prevalence of painful conditions have been found to be comparable in older adults with 
and without dementia, there is a notion that cognitive impairment is associated with reduced pain perception (Berthier, Starkstein, \& Leiguarda, 1988; Biersdorff, 1994). It is not surprising that researchers have considered the possibility of reduced pain perception with cognitive impairment, when older adults with mild dementia have demonstrated greater pain tolerance and an attenuated autonomic response to pain (e.g., heart rate, sympathetic skin response) compared to cognitively intact counterparts (Benedetti et al., 2004; Kunz, Mylius, Schepelmann, \& Lautenbacher, 2009; Rainero, Vighetti, Bergamasco, Pinessi, \& Benedetti, 2000). However, a more plausible explanation is that greater pain tolerance and reduced autonomic response to pain are due to changes to cognitive and affective processes rather than any diminishment in sensory perception (Benedetti et al., 1999; Rainero, et al., 2000). For example, the attenuated autonomic response to pain is likely due to a reduced anticipatory pain response related to decline in cognitive processes such as attention and memory. This explanation is also consistent with known neurological changes over the course of Alzheimer's disease, where primary sensory regions remain relatively intact and prefrontal, limbic and association regions show most damage (Zillmer et al., 2008).

Other researchers have investigated whether neurodegeneration leads to reduced pain perception and found little support for this notion(Benedetti et al., 2004; Gibson, Voukelatos, Ames, Flicker, \& Helme, 2001; Kunz, Scharmann, Hemmeter, Schepelmann, \& Lautenbacher, 2007). Compared to matched-controls, older adults with dementia showed comparable sensitivity to stimulus and pain thresholds (Benedetti et al., 1999). In a study involving functional magnetic resonance imaging, older adults with and without dementia showed similar pain-related brain activity (Cole et al., 2006). In particular, the 
researchers found that older adults with Alzheimer's disease showed greater activity in the sensory, affective and cognitive regions compared to cognitively intact controls. In cases of vascular dementia, when compared to healthy controls, older adults with vascular dementia self-reported greater pain, along with observed pain behaviours during activity (Scherder et al., 2003). The researchers noted that due to deafferentation (i.e., disruption of nerve signals to the central nervous system which may cause pain), those with vascular dementia may in fact be experiencing increased pain.

To date, no studies have found clinically meaningful reduction in pain sensitivity in individuals with dementia. Thus, the notion that dementia also leads to reduced pain perception is unlikely. Rather, cortical and subcortical degeneration likely lead to changes in cognitive processes which then influence verbal reporting of pain, while sensory processes responsible for pain perception are left intact. Moreover, older adults with dementia have been found to show increased non-verbal responses to painful stimulation possibly as a result of fear due to difficulty in interpreting the situation (Hadjistavropoulos, LaChapelle, MacLeod, Snider, \& Craig, 2000). As well, while many subtypes of dementia share common features of neuropathology, the extent and areas of damage and consequent effect on pain perception differ across subtypes and individuals (Scherder et al., 2003). Thus, based on the above-mentioned findings, it would be prudent to assume that all older adults with dementia do not experience clinical pain to a lesser degree than their cognitively intact counterparts.

1.4.5 Underassessment and undermanagement of pain. Reduction in selfreport of pain and underassessment of pain lead to problems in pain management. In the last decades, a great deal of evidence has accumulated to show that a disparity in 
analgesic administration exists between older adults with and without dementia (Afzal et al., 2010; Sampson, Gould, Lee, \& Blanchard, 2006). These findings have been established across various settings, including acute hospital care, palliative care, and LTC facilities. In addition, researchers have studied the disparity in pain treatment as applied to acute pain (e.g., due to fractures) and chronic pain (e.g., arthritis).

With respect to acute hospital care, researchers examined how pain was assessed for older patients with and without dementia, within 72 hours post-admission for hip fractures and surgery (Herr et al., 2004). Findings showed that pain was inadequately assessed (i.e., pain was not assessed every 2 to 4 hours post-operatively) for both older patients with and without dementia during the 72 hours post-admission for a hip fracture, a time period when pain is known to be intense and is a priority in care. Interestingly, the researchers found that while pain was inadequately assessed in both patients with and without cognitive impairments, those with cognitive impairments were more likely to be assessed behaviourally for pain. However, though nurses may have attended to facial cues to determine the presence of pain, detecting pain this way did not lead to changes in administration of analgesic (Katsma \& Souza, 2000).

Within palliative care, differences in pain management for older patients with dementia and those who are cognitively intact have been documented. Afzal and colleagues (2010) compared in-hospital end-of-life care for patients with and without dementia. It was found that those with dementia were equally likely to undergo invasive interventions but significantly less likely to be prescribed palliative drugs and palliative care interventions. Similarly, Sampson and colleagues (2006) found, based on retrospective case reviews that older patients with dementia were less likely to receive 
palliative medication and be referred to palliative care. However, in this study, patients with dementia were less likely to undergo invasive procedures, such as mechanical ventilation and central lines, compared to patients who were cognitively intact.

With regards to LTC facilities, differences in pain assessment and management have been found for residents with and without dementia (Reynolds et al., 2008). Nurses were more likely to document that cognitively intact residents experienced daily and moderate to severe pain compared to residents with dementia. However, there was no evidence based on their chart diagnoses (e.g., gout, degenerative disk disease, etc.) that residents with cognitive impairment were experiencing less painful conditions (Reynolds et al., 2008). With respect to pain intervention, cognitively intact residents were more likely to receive scheduled and stronger pain medication than those with cognitive impairment. Specifically, residents who were cognitively intact were more likely to receive scheduled pain medications, as well as stronger pain medication. Kaasalainen and colleagues (1998) published similar findings - compared to matched controls, residents with cognitive impairment were prescribed significantly fewer scheduled analgesics and were administered significantly less p.r.n medications, without differences in the likelihood of living with pain. In a revealing qualitative investigation of pain management decision-making in LTC, both nurses and physicians reported that pain assessment was a considerable barrier (Kaasalainen et al., 2007). Both nurses and physicians voiced concern regarding underassessment of residents with dementia because those residents are less likely to complain about pain. Uncertainty in diagnosing pain in residents with dementia was also a significant concern, due to the inadequacy of tools being used and difficulty in differentiating between different underlying causes of pain. 
It should also be noted that adequate assessment and treatment of pain is a challenge for LTC staff regardless of resident cognitive ability. Reynolds and colleagues (2008) found that only $24.3 \%$ of residents were charted to be experiencing pain, when research has shown that the prevalence of pain in LTC populations ranges from 50 to 80\% (Middleton, Knezacek, Robinson, Hartley, \& Kaasalainen, 1997; Weiner, Peterson, \& Keefe, 1999). Similarly, researchers have found that physicians did not detect pain in many of LTC residents who were known to live with chronic pain (Sengstaken \& King, 1993). Especially for residents who were non-communicative, physicians were less likely to detect chronic pain and to prescribe scheduled pain medication, compared to residents who were communicative. As with other studies, residents who were non-communicative did not differ on demographics or medical diagnoses other than dementia from those who were communicative.

1.4.6 Consequences of unmanaged pain. Unmanaged pain has serious negative consequences for both older adults and caregivers (Kovach, Noonan, Schlidt, \& Wells, 2005). Living with untreated pain has been linked to deterioration of resident affect, behaviour, physical status and immune function, as well as caregiver burnout, increased use of chemical and physical restraints, and reduced quality of care (Duffy, Oyebode, \& Allen, 2009; Edberg \& Hallberg, 2001; Frank, Kleinman, Ciesla, Rupnow, \& Brodaty, 2004). Findings on the impact of unmanaged pain on LTC residents and care staff are reviewed below.

One of the most apparent consequences of unmanaged pain in LTC residents with dementia is an increase in behavioural disturbances, such as disruptive behaviours that make routine care for the residents more difficult (Cipher, Clifford, \& Roper, 2006). 
Behavioural disturbances may include "physical combativeness, verbal aggression, agitation, socially disruptive behaviour, withdrawal, and wandering” (Cipher et al., 2006). Greater resident self-reported pain is also related to more intense, frequent and dysfunctional behaviours of longer duration, with this relationship becoming stronger with more severe dementia (Cipher et al., 2006). Further, behavioural disturbances and subsequent disruption to routine care may also negatively impact the resident's independence. It has been suggested that pain in older residents with dementia may not directly affect activities of daily living, but that pain leads to mood and behavioural disturbances, which in turn lead to disruption in the resident's ability to carry out activities of daily living (Cipher \& Clifford, 2004). Additionally, behavioural disturbances due to unmanaged pain can also be misinterpreted as symptoms of dementia, leaving pain untreated.

A study investigating the effect of analgesia on behavioural disturbances showed that administration of regular analgesic reduced the number of behavioural disturbances in LTC residents with dementia. Specifically, in a small sample of 10 residents with dementia and exhibiting difficult behaviour, when acetaminophen was administered regularly 3 times a day, behavioural symptoms decreased by 63\% (Douzjian et al., 1998). In fact, $75 \%$ of psychotropic medications were discontinued due to the decrease in difficult behaviours. While replication of results is necessary, these findings support the notion that behavioural disturbances may in part be due to unmanaged pain and discomfort, and with adequate pain management, can be significantly ameliorated.

While unmanaged pain leads to unnecessary suffering for LTC residents, the impact on LTC nurses and care staff cannot be overlooked. Indeed, most formal 
caregivers for residents with dementia report experiencing aspects of burnout, with 68\% of care assistants reporting emotional exhaustion, 43\% reporting depersonalization, and 25\% reporting the sense of accomplishing little in their work (Duffy et al., 2009). Most care assistants also perceived residents with dementia as "very" to "extremely challenging” to work with, with none reporting that the residents are "not challenging." Additionally, formal caregivers are vulnerable to physical assault from residents with dementia, often in the context of providing care (Morgan, Stewart, D’Arcy, Forbes, \& Lawson, 2005). Though resident aggressive behaviours were perceived as one of the most difficult aspects of care, other behaviours such as physical behaviours (e.g., wandering, inappropriate undressing) and vocal behaviours (e.g., swearing, repeating questions) were also perceived as demanding or challenging (Edberg \& Hallberg, 2001). The residents’ limited ability to communicate their needs and thus greater onus on nurses to interpret challenging behaviours likely add to work stress. Nurses reported interpreting demanding behaviours from different perspectives, ranging from signs of fear and aversion, repetition of past behaviours, to symptoms of dementia. Specifically, nurses most often perceived residents with dementia as “anxious", as "having little control over their difficult behaviour”, “unpredictable”, “lonely”, and “frightened/vulnerable” (Brodaty, Draper, \& Low, 2003). It is unclear the extent to which nurses and care aides consider untreated pain and discomfort as causes of challenging behaviours. However, if challenging behaviours were in part due to discomfort and pain, especially when exacerbated by movement in routine care, improved pain assessment and treatment will likely be beneficial (Douzjian et al., 1998). Indeed, systematic use of observational pain assessments by nurses have been found to increase the use of pain interventions, decrease 
pain behaviours observed while also reducing nurse stress and burnout (Fuchs-Lacelle, Hadjistavropoulos, \& Lix, 2008).

\subsection{Observational Pain Assessment in Older Adults with Dementia}

As discussed, a major contributor to undermanagement of pain in older adults with dementia is decline in the ability to verbally self-report pain (Oosterman et al., 2008). The reason that verbal self-report has such profound effect on pain management is that verbal self-report of pain is conventionally considered to be the gold standard of pain assessment (AGS Panel on Persistent Pain in Older Persons, 2002). However, when working with older adults with dementia and limited ability to communicate, using the verbal self-report to determine the site, intensity or duration pain may no longer be feasible or valid. Thus, research in the area of pain assessment for older adults with dementia has focused on observer identification. As such, researchers have focused on identifying pain behaviours - verbal, paraverbal (e.g., moans and cries) and nonverbal behaviours (e.g., body postures, facial expressions) that communicate to observers that pain is experienced (Fordyce, 1976; Keefe, Somers, Williams, \& Smith, 2011). Indeed, specific behaviours have been found to increase during physically demanding activity associated with pain (Hadjistavropoulos et al., 2000), and decrease when analgesia was applied (Warden, Hurley, \& Volicer, 2003). Especially important in behavioural observation of pain is identifying change from baseline behaviour (McDonald, 1999), where a resident who normally rocks may become quiet and withdrawn while in pain (Marzinski, 1991).

Focusing on observable signs of pain, the AGS Panel on Persistent Pain in Older Persons (2002) has published specific recommendations about pain assessment for older 
adults with moderate to severe dementia. It was recommended that patients be observed for movement-related pain (e.g., grimacing during personal care) as well as nonmovement-related pain (e.g., insomnia, diminished appetite), with focus on six behavioural domains of common pain cues noted in older persons with cognitive impairment. The six domains include:

(1) Facial expressions - frowning, sadness, fright or fear, grimacing, rapid blinking, and other distorted expressions that could be pain related.

(2) Verbalizations and vocalizations - sighing, moaning, groaning, grunting, chanting, calling out, noisy or laboured breathing, asking for assistance, or verbal abuse.

(3) Body movements - rigidity, tense body positions, guarding, fidgeting, increased agitation such as pacing or rocking, restricted movement, or gait/mobility changes including changes in body movements that may be indicative of pain.

(4) Changes in interpersonal interactions - increased aggression, resistance of routine care, decreased social interactions, or withdrawal from regular social activities, and disruptive behaviour.

(5) Changes in activity patterns or routines - changes in appetite, changes in sleep behaviour, increased wandering, and sudden changes in regular routines.

(6) Mental status changes - increased crying, increased confusion, irritability, and increased distress. 
While many experienced health care professionals are aware of and attend to nonverbal pain expressions in patients with dementia (Mentes et al., 2004), the need for standardized and systematic observational assessment remains. For example, people generally hold implicit and personal opinions about what they consider to be a pain behaviour or expression, thus leading to potential under- or overdiagnosing pain (Katsma \& Souza, 2000; Keefe et al., 2011; McCaffery, Ferrell, \& Pasero, 2000). In addition, judgements of pain are often influenced by the social context, where health care professionals have been found to rate infant and adult pain as lower than ratings done by family members (Kappesser, de C. Williams, \& Prkachin, 2006; Riddell \& Craig, 2007; Prkachin, Solomon, \& Ross, 2007). Moreover, untrained observers often do not attend to all available pain cues when determining other people's pain, indicated by the tendency for self-report ratings to correlate more strongly with standardized ratings than observer ratings (Eritz \& Hadjistavropoulos, 2010; Prkachin, Berzins, \& Mercer, 1994). As such, standardized observational pain tools are likely beneficial for optimizing observer pain detection.

1.5.1 Development of observational pain assessment tools. The past decades have seen a burgeoning development and application of numerous observational pain assessment tools for use with older adults with dementia (e.g., Zwakhalen et al., 2006b). Reviews of available observational pain tools for older adults with dementia, including systematic reviews, have been conducted (Herr, Bjoro, et al., 2006; Herr et al., 2010; Park, Castellanos-Brown, \& Belcher, 2009; Stolee et al., 2005; Zwakhalen, Hamers, AbuSaad, et al., 2006). Some important conclusions from reviews conducted are as follows. First, at least 39 observational pain tools have been developed for assessing pain in older 
adults with dementia, though not all tools are known to be appropriate for clinical practice or have been examined with older adults with dementia (Herr et al., 2010; Stolee et al., 2005). Secondly, all tools vary in the extent that they assess the behavioural domains suggested by the AGS Panel (2002), with eight tools containing items from five to all six of the domains (i.e., Abbey Pain Scale, Abbey et al., 2004; ADD, Kovach, Weissman, Griffie, Matson, \& Muchka, 1999; DOLOPLUS-2, Wary, 1999; PACSLAC, Fuchs-Lacelle \& Hadjistavropoulos, 2004; PADE, Villanueva, Smith, Erickson, Lee, \& Singer, 2003; DBS, Stevenson, Brown, Dahl, Ward, \& Brown, 2006; Dis DAT, Regnard et al., 2007; EPCA-2, Morello, Jean, Alix, Sellin-Peres, \& Fermanian, 2007). Third, available tools show moderate reliability and validity, though all tools require further investigation in terms of normative data, interpretation of scores, etc (Zwakhalen et al., 2006b). Finally, clinical utility of the assessment tools vary, along with item number, administration time, source of items, response style, and whether personal knowledge of the patient is needed. Based on rigorous criteria for psychometric properties (i.e., reliability and validity), comprehensiveness of scale, and clinical utility, reviewers appear to show consensus as to which tools are most appropriate for use.

1.5.2 Comparisons and reviews of observational pain tools. When compared to other observational pain tools, reviews of the PACSLAC have been very favorable (Herr et al., 2006, 2010; Lints-Martindale et al., 2012; Zwakhalen et al., 2006a; Zwakhalen et al., 2006b). In a systematic review conducted by Zwakhalen and colleagues (2006b), 12 observational pain tools were compared on psychometric properties including content validity, criterion validity, construct validity, homogeneity of items, inter-rater and intrarater reliability, as well as clinical utility. The authors concluded that amongst the 12 
tools, the PACSLAC and DOLOPLUS-2 were found to be the most appropriate scales available at the time (Zwakhalen et al., 2006b). The DOLOPLUS-2 (Wary et al., 1992) was developed based on a pain scale for young children, and adapted for assessing pain in older adults. Behaviours assessed by the DOLOPLUS-2 range from somatic, psychomotor to psychosocial reactions to pain, and the tool is unique in its assessment of an older person over time. Also, at the time of the review, the DOLOPLUS-2 had been most comprehensively tested in a variety of care settings, attesting to its clinical utility (Zwakhalen et al., 2006b). On the other hand, the PACSLAC (Fuchs-Lacelle \& Hadjistavropoulos, 2004b) assesses pain behaviours across facial expressions, activity/body movement, social/personality/mood changes, as well as other changes (e.g., trembling, sweating). According to Zwakhalen and colleagues (2006b), the PACSLAC appeared to have the ability to detect subtle changes in behaviour which would optimize pain assessment in nonverbal older adults.

In a more recent systematic review comparing 14 observational pain tools, Herr and colleagues (2010) identified the PACSLAC and the PAINAD as "the most representative of current state of science, most clinically relevant, and practically applicable to integrate into everyday practice and support adherence to regulatory guidelines.” A total of 14 tools (i.e., Abbey Pain Scale, CNPI, CPAT, DBS, Dis-DAT, DOLOPLUS-2, EPCA-2, MOBID, NOPPAIN, PACSLAC, PADE, PAINAD, PAINE, PBOICIE) were rated by experts on several categories: relevance, reliability, validity, utility, response style (i.e., yes/no), and fit with minimum data set 3.0 and other pain management standards. While the NOPPAIN, PAINAD and PACSLAC emerged as tools that performed best based on expert consensus, the NOPPAIN was judged to require 
further validation in clinical settings. For the PAINAD and PACSLAC, Herr and colleagues (2010) recommended combining their use in clinical settings, where the PACSLAC might provide a comprehensive monthly or quarterly pain screen, while the brief and more focused PAINAD might be used daily. The strengths of each tool were also highlighted, where the PAINAD has demonstrated sensitivity to change (Lane et al., 2003) and thus may be appropriate to more frequent monitoring pain interventions, while the PACSLAC's comprehensive item range increases its usefulness for completion of minimum data set assessments in LTC (Herr et al., 2010).

Currently, there are four known comparisons of observational pain tools for older adults with dementia involving the PACSLAC (Lints-Martindale et al., 2012; Liu, Briggs, \& Closs, 2010; Sheu, Versloot, Nader, Kerr, \& Craig, 2011; Zwakhalen et al., 2006a). Zwakhalen and colleagues (2006a) compared three pain tools known to have good psychometric properties (i.e., Dutch versions of the PAINAD, DOLOPLUS-2, PACSLAC). In this study, nurses and independent raters used the tools while observing videos of LTC residents with dementia undergoing painful needle injection for vaccinations. All three tools demonstrated satisfactory reliability (i.e., internal consistency, inter and intra-rater reliability), construct validity (i.e., correlation between resident self-report and observational tool scores), as well as concurrent validity (i.e., correlation among nurse rating of resident pain and observational tool scores). The DOLOPLUS-2 posed challenges in comparisons due to difference in its format (i.e., requiring more than one observation moment) compared to the PACSLAC and PAINAD. Further, LTC nurses who applied the tools in practice rated the PACSLAC as most clinically useful and quick to administer, likely due to its checklist format (Zwakhalen et 
al., 2006a). Clinical usefulness of observational pain tools has been an important factor in comparison studies involving nurses and care aides. Specifically, for observational pain assessment to be systematically feasible, features such as the checklist's length and time needed to administer the tool are critical to LTC staff (Zwakhalen et al., 2006a; Zwakhalen et al., 2006b). Regular pain assessments, although beneficial to LTC residents, could affect nurse workload and work stress. The clinical usefulness of the PACSLAC has been investigated by measuring nurse stress and burnout while nurses used the tool regularly for a three-month period (Fuchs-Lacelle et al., 2008). Results of the study revealed that regular implementation of pain assessment using the PACSLAC led to an increase in the use of p.r.n. pain medication, decrease in pain scores, and decrease in nurse stress and burnout, compared to a control group that involved nurses completing a general activity checklist for the resident. These findings suggest that regular and systematic pain assessment may lead to improved pain management, but also decrease staff stress through increasing certainty and support in pain management.

Liu and colleagues (2010) conducted a comparison study among four observational pain tools with LTC residents with osteoarthritis, also with or without dementia. The English versions of the PACSLAC, PAINAD, Abbey Pain Scale, and the DS-DAT were administered by trained observers while observing residents at rest and during exercise, and then compared on internal consistency, inter-rater reliability, as well as discriminant, convergent and concurrent validity. All four scales were said to show moderate to good psychometric properties (i.e., internally consistent, ability to discriminate between exercise and at rest, etc.), especially in the exercise condition compared to the at rest condition. The authors concluded that all scales showed similar 
reliability and validity, suggesting that they are appropriate for use with residents with dementia experiencing exercise-exacerbated chronic pain (Liu et al., 2010).

In contrast to other comparison studies, Sheu and colleagues (2011) compared the extent to which observational pain tools (i.e., PACSLAC, DOLOPLUS-2, Mahoney, Abbey, NOPPAIN, PAINAD) correlated with scores from the Facial Action Coding System (Ekman \& Friesen, 1978). The FACS is an anatomically-based observational coding system that measures all visually discernible facial movements (Rosenberg, 2005). This is done by identifying the actions of specific facial muscles or groups of facial muscles that are involved in facial expressions. Slow motion video footage of individuals' faces are viewed by trained observers and coded into facial movements, or action units. A total of 44 action units have been identified, which are fine facial movements (e.g., raised inner brow) that involve the action of one or more facial muscles (e.g., frontalis, pars medialis). Facial expressions displayed along with specific emotions can then be described in terms the actions units involved. This fine-grained observational coding system is used mainly in experimental settings due to the time-intensive process of frame-by-frame coding, but has allowed researchers to describe facial expressions in an objective manner. Compared to other observational coding systems, the FACS is considered to be more comprehensive and objective because it codes all discernible facial movements, while other systems are more selective (Rosenberg, 2005). Research using the FACS has extended to pain research where a core constellation of action units have been identified to be more likely to occur when pain is experienced and expressed. In the comparison study, Sheu and colleagues (2011) coded video footage of older hospital patients during physical activity. Notably, the sample involved older patients with and 
without cognitive impairment: 32\% no impairment, 22\% mild impairment, 25\% moderate and $22 \%$ severe impairment. Intensity scores from facial action units most commonly associated with a pain expression (i.e., lowered brow, raised cheek, raised upper lip, closed eyes) were used to categorize mild, moderate and severe pain levels observed in the patients. Independent coders then used the observational pain tools while viewing the patient video footage, which allowed for comparison amongst the tools and with FACS intensity scores. Among the observational pain tools, the Abbey, Mahoney and the PACSLAC showed significantly different mean scores across the mild, moderate and severe pain levels. Further, tool scores were correlated with FACS scores at each pain level. It was found that the PAINAD and Mahoney correlated with FACS at all three levels, and the PACSLAC, NOPPAIN, and Abbey correlated with FACS at the moderate and severe pain levels. The DOLOPLUS-2 correlated with FACS at the mild level but the relationship was inverse. Notably, observational pain tools also showed lower inter-rater reliability at the mild pain level. It may be that these tools are less sensitive than objective measurement of facial pain expression when pain is at the mild level. The authors suggest that facial expression items in existing observational pain tools may not have adequate construct and concurrent validity (Sheu et al., 2011). In particular, they indicated that the presence of facial expression items (e.g., sad look, frightened look) representing other emotions and imprecisely worded items may lead to decreased reliability and construct validity.

Most recently, Lints-Martindale, Hadjistavropoulos, Lix and Thorpe (2012) compared 6 observational pain tools using the same sample of older adults with dementia experiencing acute and movement-exacerbated chronic pain. Video footage of LTC 
residents with moderate to severe dementia were taken while at rest, during needleinjection for influenza immunization, and during routine activity involving movement (e.g., physiotherapy, transfers, daily care). Trained research assistants coded the videos using 6 tools that were selected based on their coverage of AGS behavioural domains and expert consensus (Hadjistavropoulos et al., 2007). Tools included the PACSLAC (FuchsLacelle \& Hadjistavropoulos, 2004), the PADE (Villanueva et al., 2003), the PAINAD (Warden et al., 2003), the ADD (Kovach et al., 1999), the CNPI (Feldt, 2000), and the NOPPAIN (Snow et al., 2004). A number of important results were found. All tools demonstrated significant difference between pain and baseline scores, convergent validity (i.e., correlation among the tools) and discriminant validity (i.e., no correlation between tools and a measure of depression). Amongst the tools, the PACSLAC and the PAINAD demonstrated the highest internal consistency when measuring both needle injection and movement-exacerbated pain conditions (i.e., $\alpha=.79$ to .73 ). Further, researchers examined the effect of removing tool items based on their indication of delirium (i.e., an acute and transient disturbance in consciousness and cognition due to underlying physiological changes which overlaps in symptom presentation with pain in this population). Once delirium-related items (e.g., agitated) were omitted, it was found that all tools, except for the ADD, retained the ability to discriminate between baseline and pain conditions. This suggests that it may be possible to assess pain in older people with dementia, while guarding against the symptom overlap between delirium and pain. Moreover, Lints-Martindale and colleagues (2012) analyzed each tool's ability to differentiate between pain and baseline conditions while controlling for the contribution of all other tools. In order to test this, the researchers calculated difference scores 
between each pain and baseline condition (with and without the removal of deliriumrelated items), and entered these scores as covariates in the analyses, thus controlling for the variance accounted for by other tools. Results showed that the PACSLAC was the only tool able to differentiate between each pain condition and baseline, after controlling for the contribution of other tools, with and without delirium-related items. The NOPPAIN, without delirium-related items, differentiated between only movementexacerbated pain and baseline when controlling for the contribution of other tools. The PADE with and without delirium-related items, differentiated between only needleinjection pain and baseline when controlling for the contribution of other tools. These findings indicate that certain tools may show greater ability to differentiate between pain and non-pain states than others, and that the PACSLAC, with more comprehensive coverage of AGS behavioural domains, may show greater sensitivity to pain (LintsMartindale et al., 2012).

Thus, given the positive findings on the PACSLAC's reliability, validity and clinical utility, combined with evidence that symptoms overlapping with delirium may not be needed, we determined that further refinement and validation are warranted. The following will be a review of the work conducted on the PACSLAC to date, including its initial development, as well as empirical and theoretical information relevant to its subsequent revisions.

1.5.3 Development of the PACSLAC. The PACSLAC (Fuchs-Lacelle \& Hadjistavropoulos, 2004) was originally constructed with the purpose of creating a clinically useful observational pain tool, specifically to be used with older adults with dementia and limited ability to communicate. While similar tools existed at the time of its 
development, the PACSLAC was aimed to be more comprehensive and psychometrically sound. Item generation and scale development of the PACSLAC involved semistructured individual interviews with LTC nurses experienced in working with residents with dementia (Fuchs-Lacelle \& Hadjistavropoulos, 2004). A list of pain behaviours was generated from interview transcripts, and then consolidated by collapsing words that were determined by the researchers to have similar meaning. For example, screaming was combined with yelling/hollering, to create screaming/yelling. A total of 65 items were then organized into conceptually-based subscales and formatted as a checklist with conceptually organized subscales of Facial Expressions, Aggressive Behaviours, Vocal Behaviours, Activity/Body Movements, Eating/Sleep Changes, Physiological Indicators, and Social/Personality/Mood Indicators. In the second phase of in the development of the PACSLAC, another group of nurses completed the checklist retrospectively in reference to recalling a recent patient with dementia who was experiencing pain. The nurses also rated the usefulness of each item to examine the items' face validity and content validity. Nurses' ratings of item usefulness also indicated that items were adequate to excellent on clinical usefulness.

In the third phase of development, the PACSLAC's ability to discriminate between pain, non-painful distress and baseline conditions was examined. Nurses were asked to recall and rate, using the PACSLAC, four different events from one patient with severe dementia: two pain events in which pain was clearly the cause, one non-painful distress event (e.g., where the patient's family was leaving) and one event in which the patient was calm. Concurrent validity was supported by correlating the pain intensity scores and PACSLAC total scores. Validity was supported by demonstrating that scores 
differed significantly across the pain event, non-painful distress and calm event, suggesting that items differentiated between pain and no pain states. Data collected from this phase also aided the elimination of items that did not discriminate pain from a calm event (e.g., sitting and rocking, vacant blank stare, quiet/withdrawn). With elimination of items, the PACSLAC had a total of 60 items, showed excellent internal consistency in its total score, and acceptable to very good internal consistencies in subscales scores, and took approximately five minutes for nurses to complete.

In a follow-up study for further validation of the PACSLAC, nurses completed the tool while working with LTC residents with dementia, thus collecting prospective data for the baseline condition (Fuchs-Lacelle \& T. Hadjistavropoulos, 2005). Previously collected total scores for the pain condition and non-painful distress condition, were compared to the total at-rest scores (i.e., collected from nurse observation over the course of an 8-hour shift), and were found to be significantly different, thus supporting the PACSLAC's ability to discriminate among residents in pain, in distress and at rest. Moreover, as stated earlier, in a prospective longitudinal study, Fuchs-Lacelle and colleagues (2008) confirmed the clinical usefulness of the PACSLAC.

1.5.4 Further Research on the PACSLAC. Aside from the original authors, other researchers have also studied the PACSLAC when implemented in practice with nurses and LTC residents with dementia (Aubin et al., 2008; Cheung \& Choi, 2008). Cheung and Choi (2008) found, using the English version of the PACSLAC, that the total PACSLAC score demonstrated good inter-rater reliability when used by a researcher and a formal caregiver for residents in special dementia care home. Using the French version of the PACSLAC, Aubin and colleagues (2008) investigated its psychometric properties 
by having LTC nurses observe and assess 86 residents with severe dementia, and in calm, painful and non-painful distress states. Satisfactory internal consistency, test-retest reliability, discriminant validity and convergent validity (i.e., with the DOLOPLUS-2) were found, further validating the use of the PACSLAC with this population.

As mentioned, a group of researchers based in the Netherlands also conducted a series of investigations involving the Dutch version of the PACSLAC (van Nispen tot Pannerden, Candel, Zwakhalen, et al., 2009; Zwakhalen, Hamers, \& Berger, 2007; Zwakhalen et al., 2006a). Aside from the aforementioned comparison study among Dutch versions of the PACSLAC, PAINDAD and DOLOPLUS-2, which provided evidence for the reliability and validity of the tools, their work on the PACSLAC-D (Zwakhalen et al., 2007) focused on the analysis of factor structure, and reduction of items using both classical test theory and item response theory methods.

Two factor analyses were conducted with the aim of item reduction, and were based on a sample of archival video footage (van Nispen tot Pannerden, Candel, Zwakhalen, et al., 2009; Zwakhalen et al., 2007). The video data consisted of a total of 290 segments, with 128 segments of rest, 127 segments of painful needle injection for influenza vaccination, and 35 segments of movement-exacerbated pain (e.g., washing, transfer, hand manipulation and wound care (Zwakhalen et al., 2007). Based on these data, items that were endorsed over $90 \%$ of the time and less than $10 \%$ of the time were culled, in order to eliminate floor and ceiling effects. Items with less than .20 of the itemtotal correlation were also eliminated. The remaining items were entered into a principal component analysis and yielded a three-component structure, which the researchers identified as Facial and Vocal Expressions, Resistance/Defense, and Social-emotional 
aspects/Mood. A second factor analysis was also conducted as part of a reduction of the PACSLAC-D based on both classical test theory and item response theory techniques (van Nispen tot Pannerden et al., 2009a). Again, items were eliminated based on ceiling and floor effects, low contribution to total correlation, and factor loadings less than .40 and other criteria. The three factors were identified as Negative reactions to pain, Facial/Vocal/Emotional expressions of pain, and Resistance to pain. The shortening of the Dutch version of the PACSLAC may be methodologically problematic in that it resulted in the elimination of items based on endorsement rates and on a single sample of video footage. For instance, depending on the characteristics of the sample of video data, biases may result in scale revisions based on these data. That is, the sample of video data of pain events employed by Zwakhalen and colleagues (2007) was composed largely of pain moments during needle injection, which may disproportionately represent a very specific type of phasic pain. Thus, it is possible that revisions of items based on data biased toward needle injection pain would prematurely eliminate items that reflect movement-exacerbated pain.

Using item-response techniques, van Nispen tot Pannerden and colleagues (2009) conducted other examinations on the shortened Dutch version of the PACSLAC, including analysis of item bias, as well as establishing a cut-off score. The item frustrated was found to be more likely to be used with patients with severe dementia regardless of pain level, and pain expression was more likely to be used with patients with less severe dementia. Cheung and Choi (2008) also found possible item bias in the English version of the PACSLAC, where total scores increased with increasing dementia severity. At the same time, there is evidence that older adults with dementia may exhibit 
more pronounced pain behaviours (e.g., facial expressions) compared to those without cognitive impairment (Kunz et al., 2007). It is for this reason that the developers of the PACSLAC have recommended the individualized approach to observational pain assessment (T. Hadjistavropoulos, Fitzgerald, \& Marchildon, 2010).

With respect to a cut-score, van Nispen tot Pannerden and colleagues (2009) compared the full, Dutch version of the PACSLAC and shortened Dutch PACSLAC on their ability correctly judge when an individual is in a moderate level of pain, and calculated a cut-score using receiver operational characteristic curves. However, given the aforementioned issues regarding possible sampling bias in the study (e.g., based on needle injection data only), it may be premature to conclude that a cut-off score is appropriate for application in clinical settings. As well, dementias that are present in clinical settings are varied, and research is beginning to show that pain experience differs across Alzheimer's disease, vascular dementia and frontotemporal dementia (Scherder et al., 2003). Therefore, since using a cut-off score to suggest clinical intervention has serious consequences for the patient, the use of a PACSLAC cut-off score would require further validation involving greater understanding about the differences in pain experience among dementia types, as well as the establishment of normative scores (Kellow, \& Wilson, 2008). Moreover, scores may also vary depending on factors such as conditions and duration of administration.

In contrast, the authors of the PACSLAC have recommended an individualized approach to using the instrument, whereby a baseline series of scores is collected for each resident against which future assessments can be compared (Hadjistavropoulos, 2007 as cited in Regional Geriatric Program Central, 2010). Cheung and Choi (2008) also 
suggested a similar approach, where a baseline PACSLAC score can be performed for each LTC resident with dementia upon admission, and then re-assessed at regular intervals in order to monitor pain management.

In summary, research on the PACSLAC to date has demonstrated strengths in its psychometric properties and clinical utility when compared to other tools of its kind. While revisions have been conducted with the Dutch version of the tool, these revisions were guided by item endorsements and other statistical techniques, and did not incorporate theoretical and empirical literature about pain expression. For instance, it has been suggested that, in existing observational pain tools developed through staff narratives, that facial expressions subscales may include facial expressions that are commonly assumed to be indicative of pain, but are not supported by more objective measures (Craig, Prkachin, \& Grunau, 2011). Colloquially-worded items derived from interviews may also lack specificity and could potentially lead to difficulty in identification of pain. More recent comparison between objective measurement of pain facial expressions and pain observational tools show that facial expression items in these screening tools may lack in construct validity (Sheu et al., 2011). In addition, recent work has shown that scores on pain assessment tools may be inflated as a result of delirium, since certain pain behaviours and symptoms of delirium overlap (T. Hadjistavropoulos, Voyer, Sharpe, Verreault, \& Aubin, 2008). As mentioned, Lints-Martindale and colleagues (2012) have shown that elimination of delirium-related items for these scales, and especially the PACSLAC, did not have a major effect in psychometric properties. Such findings, together with the AGS guidelines for pain assessment in dementia, suggested the need for a systematic and empirically-driven revision of the PACSLAC. 
The theoretical and empirical literature that guided the PACSLAC revisions are reviewed.

\subsection{Review of Research on Pain Behaviours}

With regards to observational assessment of pain, Keefe and colleagues (2011) made the following recommendations for selecting observable cues to watch for. First, pain cues should occur exclusively when pain is present, and not when pain is absent. Second, the cue should occur with sufficient frequency such that it is likely to be observed. Third, the cue should not be difficult to elicit during daily tasks. Fourth, the cue should be reliably observed, such that different observers could agree upon the definition of the pain behaviour and also when it has occurred. In this regard, much research has accumulated regarding cues exhibited by individuals in pain, including older adults with dementia. In particular, the behavioural domains of facial expressions and body movements have gained most research attention. The following sections will review the literature on pain cues based on the pain behaviour domains suggested by the AGS panel.

1.6.1 Facial expressions. Facial expression has been acknowledged as an essential and universal component of pain communication (Craig et al., 2011a). Observers appear to be capable of discerning pain in another based on facial expressions without formal training, and certain facial expressions have been found to uniquely convey pain across sex, age, and culture (Ekman, 1993; Kunz, Gruber, \& Lautenbacher, 2006; Kunz, Mylius, Schepelmann, \& Lautenbacher, 2008). With specific relevance to the present study, facial expressions of pain have also been investigated in various populations with limited ability to communicate, including neonates, individuals with cognitive challenges, as well as older adults with dementia (Defrin, Lotan, \& Pick, 2006). 
As mentioned, the most well-established method of measuring facial expressions is the FACS (Ekman \& Friesen, 1978) and has been used to identify facial movements that occur when experiencing pain. A number of core facial muscle movements have been observed across various pain situations, including acute clinical and experimental pain, and the exacerbation of a chronic pain condition. These include: lowered brow (Action Unit [AU] 4), raised cheek (AU6), tightened eyelids (AU7), wrinkled nose (AU9), raised upper lip (AU10), lip corner pull (AU12), stretched lip (AU20), parted lips (AU25), jaw drop (AU26), mouth stretch (AU27), closed eyes (AU43), blinking (AU45) (Craig \& Patrick, 1985; Craig, Hyde, \& Patrick, 1991; Hadjistavropoulos, LaChapelle, Hadjistavropoulos, Green, \& Asmundson, 2002; Kunz, Gruber, \& Lautenbacher, 2006; Kunz, Mylius, Scharmann, Schepelman, \& Lautenbacher, 2009; Kunz, Mylius, Schepelmann, \& Lautenbacher, 2008, 2009; Kunz, Scharmann, Hemmeter, Schepelmann, \& Lautenbacher, 2007; Patrick, Craig, \& Prkachin, 1986; Prkachin, 1992, 2005; Simon, Craig, Gosselin, Belin, \& Rainville, 2008; see Table 1.1 for list of pain facial actions found using the FACS). Other features that have also been found to occur during pain are sudden jerking and eyes moving left, right, up and down (Craig et al., 2011; LintsMartindale, Hadjistavropoulos, Barber, \& Gibson, 2007). Narrowing down the list of facial movements associated with pain, Prkachin (2005) argued that, although a number of action units may be seen during different pain modalities (i.e., cold, heat, electric, muscle ischemia), there are four muscle groups and associated action units that are active most consistently across different modalities. Specifically, the researcher suggested that one may focus on lowered brow, raised cheek/tightened eyelids, wrinkled nose/raised upper lip, and closed eyes as facial actions most representative of a pain expression. Also, 
Table 1.1 Facial actions associated with pain based on FACS

\begin{tabular}{|c|c|c|}
\hline Study & Sample & Facial actions associated with pain \\
\hline $\begin{array}{l}\text { Craig \& Patrick, } \\
1985\end{array}$ & $\begin{array}{l}\text { Adult females during } \\
\text { cold pressor test }\end{array}$ & $\begin{array}{ll}\text { - } & \text { raised cheeks } \\
\text { - } & \text { tightened eyelids } \\
\text { - } & \text { raised upper lip } \\
\text { - } & \text { lip corner pull } \\
\text { - } & \text { lips parting } \\
\text { - } & \text { opened mouth } \\
\text { - } & \text { closed eyes } \\
\text { - } & \text { blinking }\end{array}$ \\
\hline Patrick et al., 1986 & $\begin{array}{l}\text { Adult females during } \\
\text { electrical stimulation }\end{array}$ & $\begin{array}{ll}\text { - } & \text { raised cheeks } \\
\text { - } & \text { tightened eyelids } \\
\text { - } & \text { raised upper lip } \\
\text { - } & \text { lip corner pull } \\
\text { - } & \text { lips parting } \\
\text { - } & \text { opened mouth } \\
\text { - } & \text { closed eyes } \\
\text { - } & \text { blinking } \\
\text { - } & \text { lowered brows }\end{array}$ \\
\hline Craig et al., 1991 & $\begin{array}{l}\text { Chronic low back } \\
\text { pain patients during } \\
\text { range of motion } \\
\text { exercises }\end{array}$ & $\begin{array}{ll}\text { - } & \text { lowered brows } \\
\text { - } & \text { raised cheeks } \\
\text { - } & \text { tightened eyelids } \\
\text { - } & \text { raised upper lip } \\
\text { - } & \text { opened mouth } \\
\text { - } & \text { closed eyes }\end{array}$ \\
\hline Prkachin 1992 & $\begin{array}{l}\text { Healthy adults during } \\
\text { different stimuli } \\
\text { (electrical, cold, } \\
\text { pressure, muscle } \\
\text { ischemia) }\end{array}$ & $\begin{array}{ll}\text { - } & \text { lowered brow } \\
\text { - } & \text { tightened eyelids } \\
\text { - } & \text { closed eyes } \\
\text { - } & \text { wrinkled nose } \\
\text { - } & \text { raised upper lip }\end{array}$ \\
\hline $\begin{array}{l}\text { Hadjistavropoulos et } \\
\text { al., } 2002\end{array}$ & $\begin{array}{l}\text { Post-knee surgery } \\
\text { older patients } \\
\text { undergoing } \\
\text { standardized } \\
\text { physiotherapy }\end{array}$ & $\begin{array}{ll}\text { - } & \text { raised brow } \\
\text { - } & \text { lowered brow } \\
\text { - } & \text { raised cheeks } \\
\text { - } & \text { lip corner pull } \\
\text { - } & \text { raised chin } \\
\text { - } & \text { puckered lips } \\
\text { - } & \text { lip stretch } \\
\text { - } & \text { lip press } \\
\text { - } & \text { lips part } \\
\text { - } & \text { jaw drop }\end{array}$ \\
\hline
\end{tabular}


- blinkng

Kunz et al., 2004

Kunz et al., 2006

Kunz et al., 2007

Lints-Martindale et al., 2007

Kunz et al., 2008
Adults during pressure and electrical stimulation

- lowered brow (pressure pain)

- raised brow (electrical pain)

- raised cheek

- tightened eyelids

- wrinkled nose

- $\quad$ raised upper lip

- lip corner pull

- opened mouth

- blinking

Males and females during heat stimulation

Older patients with dementia and healthy controls during pressure stimulation

Older adults with Alzheimer's Disease and controls, during mechanical and electrical stimulation

- lowered brow

- tightened eyelids

- wrinkled nose

- closed eyes

- lowered brow

- tightened eyelids

- closed eyes

- wrinkled nose

- $\quad$ raised upper lip

- lowered brow

- tightened eyelids

- parted lips

- jaw drop
- $\quad$ eyes moving left/right/up/down

- sudden jerk

- blinking

- closed eyes

$$
\begin{array}{lll}
\text { Younger and older } & - & \text { lowered brow } \\
\text { adults during pressure } & - & \text { raised cheeks } \\
\text { and electrical } & - & \text { tightened eyelids } \\
\text { stimulation } & - & \text { wrinkled nose } \\
& - & \text { raised upper lip } \\
& - & \text { blinking }
\end{array}
$$

Older adults with
mild cognitive
impairment, healthy
older controls,
younger adults;
during electrical
stimulation

- tightened eyelids

- closed eyes

- wrinkled nose

- $\quad$ raised upper lip

- blinking (in older adults with MCI \& healthy older controls) 
Kunz et al., 2009

Older patients with $\quad$ - lowered brow dementia and healthy - tightened eyelids controls during - closed eyes electrical stimulation - wrinkled nose

- $\quad$ raised upper lip

- blinking 
of the four movements, raised cheek/tightened eyelids and wrinkled nose/raised upper lip accounted for the greatest amount of variance (Prkachin, 2005).

Further supporting the validity of these facial muscle movements as indicative of pain, individuals who are cognitively intact tend to display more intense facial expression along with higher self-report of pain severity, though this finding will require greater replication (Craig et al., 2011). In addition, facial expression of pain reduces when pain medication is adjusted. Facial actions that frequently occur with pain have also been found to be different from other facial actions that occur with other aversive, non-painful emotions. Observers do not need training to identify facial expressions as pain-related, and can also discriminate between facial expressions for pain compared to facial expression from other aversive, non-painful emotions. Finally, untrained observers' evaluation of pain is correlated with the intensity of the pain stimulus, as well as the objectively evaluated facial expression using FACS (Craig et al., 2011).

With respect to older adults with dementia, observing facial expressions for pain in clinical settings may be especially relevant. For instance, Husebo and colleagues (2009) used a pain scale which takes into account facial expressions, pain noises and defence behaviours in residents with severe dementia during guided movement tests, and found that facial expressions were observed most. Similarly, by drawing attention to facial expressions and vocalizations, medical students and nurses were able to accurately identify the presence of pain from video footage of older persons with severe dementia undergoing painful dressing changes (Manfredi, Breuer, Meier, \& Libow, 2003; Solomon, Prkachin, \& Farewell, 1997). However, inferring the intensity of pain experience based on observation remains challenging, as suggested by low observer 
agreement about the individual's pain intensity. Researchers have also found that facial actions that occur with pain are not expressed in a uniform manner across pain modalities (Ekman, 1993). For example, when pain was induced by electrical stimulation, facial muscle movements showed an all-or-none pattern, with a large difference found between facial muscle movements between "no pain" to "pain" and a plateauing of frequency with further increases in pain. On the other hand, when pain was induced by mechanical pressure, a more gradual increase in frequency of facial muscle movement was found with increase in pressure. Taken together, these findings suggest that it may be premature to attempt to conclude based on frequency of specific facial muscle movement, the intensity of pain experienced. Rather, the presence of various facial muscle movements related to pain should be taken as suggesting presence of pain.

Based on the large body of evidence on specific facial movements frequently implicated in expressions of pain, revisions to the PACSLAC involved the incorporation of these indicators. Specifically, facial movements found to occur most consistently when individuals experience pain, such as lowered brow, raised cheek/tightened eyelids, wrinkled nose/raised upper lip, and closed eyes, were incorporated into the PACSLACII. Facial expression items on the PACSLAC that are not specific to pain, such as "sad look" and "dirty look," were removed in order to create a more specific measure. Revision of the item pool by addition, removal or rewording of items was intended to improve the validity of the facial expression subscale.

1.6.2 Verbalizations and vocalizations. Verbal and paraverbal pain cues have consistently been part of observational pain assessments, and are behaviours recommended for assessment in older adults with dementia and limited ability to 
communicate (AGS Panel on Persistent Pain in Older Persons, 2002). However, compared to other pain behaviours (i.e., facial expressions and body movements), vocalizations of pain have received comparably less research attention. Thus, a review of the literature on verbalizations and vocalizations that follows will help indicate the extent to which the PACSLAC reflects current knowledge of this behavioural domain.

Vocalizations of LTC residents with dementia have most frequently been examined in the context of a range of "disruptive" behaviours (Cariaga, Burgio, Flynn, \& Martin, 1991; Miller et al., 2000; Sloane et al., 1997). Though knowledge about vocalizations in dementia is currently based on descriptive and correlational studies, these studies do suggest that certain paraverbal behaviours are associated with pain. In particular, screams and groans, as well as volume of vocalizations appear to be associated with pain (Miller et al., 2000; Sloane et al., 1997). In a study comparing LTC residents who showed verbal agitation and those who did not, it was found that those who showed verbal agitation were less likely to be prescribed acetaminophen regularly or p.r.n. (i.e. $7 \%$ of vocalizers versus $44 \%$ non-vocalizers were prescribed acetaminophen), despite comparable prevalence of chronic pain in the two groups (Cariaga et al., 1991). Similarly, when Sloan and colleagues (1999) attempted to characterize LTC residents who were "severely disruptive vocalizers" (i.e., "someone who screams, moans, makes vocal noises, or talks loudly for at least 2 hours a day"), some trends related to pain were observed. Twenty-three percent of residents who nurses considered as severely disruptive vocalizers experienced daily pain, and most had a diagnosis of dementia (82\%), with more than half whose vocalizations were characterized by care staff as being caused by pain (60\%). Unfortunately, no information was available from this study describing the 
vocalizations thought to be indicative of pain (Sloane et al., 1999). It appears that, thusfar, knowledge on verbal and paraverbal pain behaviours is limited. This may be related to the tendency for vocalizations to be examined as part of "disruptive" behaviours in general and associated methods of intervention, rather than aiming to specify vocalizations that indicate pain. At present, description of vocalizations indicative of pain are based on consensus or LTC staff reports. Within observational pain tools, vocalizations have been described as “spontaneous utterances that relate to the patients' pain”, “moaning, sighing, puffing or slowing exhalation of breath” (Prkachin, Hughes, Schultz, Joy, \& Hunt, 2002), or simply as “ouch” or “ow” (Fuchs-Lacelle \& Hadjistavropoulos, 2004). A list of descriptors for paraverbal and verbal pain expression as used in pain behaviour assessments can be found in Table 1.2, which includes painrelated vocalizations from pain coding systems (e.g., PBM) as well as observational pain tools (e.g., PACSLAC). This information was taken into consideration when developing the verbalization and vocalization items of the PACSLAC-II.

1.6.3 Body movements. The systematic study of bodily movements indicating pain began with Keefe and Block’s (1982) operationalization of behaviours exhibited by patients with chronic low back pain. The Pain Behaviour Measurement system (PBM; (Keefe \& Block, 1982) was developed based on pain behaviours gathered by the authors from individuals familiar with patients with chronic low back pain, such as family members, nurses and physicians. Five pain behaviours were selected (i.e., guarding, bracing, rubbing, grimacing, and sighing), which were then used to code patient behaviour during standardized movements (i.e., sit, stand, walk, recline, etc.). A series of studies were subsequently conducted demonstrating the PBM's reliability, convergence 
Table 1.2 Verbalizations and vocalizations associated with pain.

Study Verbalizations and vocalizations associated with pain

Keefe \& Block, 1982 (PBM)

Prkachin et al., 2002

Follick et al., 1985

Hurley et al., 1992 (DS-DAT)
1. Sighing: obvious exaggerated exhalation of air usually accompanied by shoulders first rising and then falling and sometimes accompanied by the expansion of cheeks

1. Words: any spontaneous utterance that relates to the patients pain.

2. Sounds: moaning, sighing, puffing or slow exhalation

1. Limitation Statements: statements relating to disability or impairment, expresses inability, verbalizes hesitation, or questions capacity to perform tasks

2. Sounds: any pronounced utterance that is not language moan, groan, or grunt

1. Negative Vocalization: noise or speech with a negative or disapproving quality; hushed low sounds such as constant muttering with a guttural tone; monotone, subdued, or varying pitched noise with a definite unpleasant sound; faster rate than a conversation or drawn out as in a moan or groan; repeating the same words with a mournful tone; expressing hurt or pain

Abbey et al., 2002 (Abbey Pain Scale)

Wary et al. (DOLOPLUS-2)

Snow et al., (NOPPAIN)

Warden et al., 2003 (PAINAD)

Feldt et al., 2000 (CNPI)
1. Vocalization: e.g. whimpering, groaning, crying

1. Somatic reactions: the patient expresses pain by word, gesture, cries, tears or moans

2. Communication: verbal or non-verbal

1. Pain Words: "that hurts!", “Ouch!”, cursing, "Stop that!”

2. Pain Noises: moans, groans, grunts, cries, gasps, sighs

1. Negative Vocalization: occasional moan or groan, low-level speech with a negative or disapproving quality, or repeated troubled calling out, loud moaning or groaning, crying

1. Vocal Complaints: nonverbal (sighs, gasps, moans, groans, cries)

2. Vocal Complaints: verbal (words expressing discomfort or pain e.g., "ouch", "that hurts"; cursing during movement; exclamation of protest, e.g., "stop”, “that's enough”) 
Kovach et al., 2002 (ADD)

Cervo et al., 2007 (CPAT)

Husebo et al., 2007 (MOBID)

Cohen-Mansfield, 2006 (PAINE)

Tsai et al., 2008 (PBOICIE)

Morello et al., 2007 (EPCA-2)
1. Voice: moaning, mumbling, chanting, grunting, whining, calling out, screaming, crying, verbally aggressive

1. Behaviour: calling out/moaning

1. Pain noises: ouch!, groaning, gasping, screaming

1. Specific repetitive behaviours (vocal): moaning, grunting, groaning, gasping, sighing, crying, whimpering, whining, screaming, yelling

1. Audible expression of distress: sigh, moan, grasp, groan, words expressing discomfort or pain, "ouch", "that hurts"; cursing during movement; and exclamations of protest, "stop", "that's enough"

1. Complaints voiced in the course of caregiving: complains if the caregiver talks/as soon as the caregiver is present, moans or cries silently and spontaneously, shouts or complains violently and/or spontaneously 
with patient pain ratings, discrimination between pain and no-pain patient groups, sensitivity to treatment effects, and construct validity (F. J. Keefe \& Smith, 2002; F. J. Keefe et al., 1987, 2011). Like the PBM, other studies coding pain-related body movements also tended to involve the use of video recordings and real-time observations by trained observers using manualized coding, in order to ensure reliability and validity of pain indicators (Prkachin, Hughes, Schultz, Joy, \& Hunt, 2002).

Similar systems have been developed, including the pain behaviour assessment tools of Follick and colleagues (1985), Jensen and colleagues (1989), Prkachin and colleagues (2002), amongst others. These tools differ in the range of pain cues targeted, the maneuvers used to elicit them, and the degree that they have been tested through realtime observation, video coding, or application in clinical settings (Prkachin et al., 2002). These tools also show significant overlap across the body movements that are assessed. Studies selected based on their use of these observational assessment tools, rather than descriptive study of pain body movements, are summarized in Table 1.3.

Interestingly, Keefe and colleagues (1990) have demonstrated that among individuals with chronic pain, there seem to be clusters of patients that tend to express pain similarly through body movements. The researchers revealed four subgroups within patients with low back pain: (1) those with low levels of guarding, bracing and rubbing, (2) a high level of guarding, (3) high levels of guarding, bracing and rubbing, and (4) high levels of rubbing. Different body movements may also be expressed depending on the activity the individual is engaged in. For instance, in older patients who had recently undergone knee surgery, performing knee bends and quadricep exercises led to greater displays of rigidity, compared to reclining, standing or walking (Fuchs-Lacelle et al., 
Table 1.3 Body movements associated with pain.

\begin{tabular}{|c|c|c|}
\hline Study & Sample & Body movements indicating pain \\
\hline $\begin{array}{l}\text { Keefe \& } \\
\text { Block, } \\
1982\end{array}$ & $\begin{array}{l}4 \text { samples of } \\
\text { adults (mean age } \\
=41 \text { years) with } \\
\text { chronic low back } \\
\text { pain; coding of } \\
\text { video of } \\
\text { standardized } \\
\text { movements }\end{array}$ & $\begin{array}{l}\text { 1. Guarding: abnormally stiff, interrupted, or rigid } \\
\text { movement while walking or moving from one } \\
\text { position to another } \\
\text { 2. Bracing: stationary position in which a fully } \\
\text { extended limb supports and maintains an abnormal } \\
\text { distribution of weight, held for at least } 3 \text { seconds } \\
\text { 3. Rubbing: touching, rubbing, or holding the } \\
\text { affected area of pain for a minimum of } 3 \text { seconds. }\end{array}$ \\
\hline $\begin{array}{l}\text { Follick et } \\
\text { al., } 1985\end{array}$ & $\begin{array}{l}71 \text { adults (mean } \\
\text { age = } 38 \text { years) } \\
\text { with chronic low } \\
\text { back pain; coding } \\
\text { of video of } \\
\text { standardized } \\
\text { movements }\end{array}$ & $\begin{array}{l}\text { 1. Guarded Movement: slow, cautious movement } \\
\text { relative to baseline; nonmethodical or jerky } \\
\text { movement } \\
\text { 2. Bracing: pronounced use of extremity on body or } \\
\text { object for support; assists movement by leaning on } \\
\text { or pushing off chair, wall, body, and so forth; } \\
\text { tension or rigidity evident in weight bearing } \\
\text { extremity } \\
\text { 3. Positions Shifts: changes in body alignment or in } \\
\text { distribution of body weight } \\
\text { 4. Partial Movement: limited range of motivation, } \\
\text { does not complete movement. }\end{array}$ \\
\hline $\begin{array}{l}\text { Anderson } \\
\text { et al., } 1987\end{array}$ & $\begin{array}{l}53 \text { adults (mean } \\
\text { age }=49 \text { years) } \\
\text { with rheumatoid } \\
\text { arthritis; coding } \\
\text { of video of } \\
\text { standardized } \\
\text { maneuvers }\end{array}$ & $\begin{array}{l}\text { 1. Guarding: definition as Keefe \& Block, } 1982 \\
\text { 2. Bracing: definition as Keefe \& Block, } 1982 \\
\text { 3. Rigidity: excessive stiffness of an affected joint or } \\
\text { body part (with the exception of fingers and toes) } \\
\text { that is not directly involved in locomotion. } \\
\text { Rigidity can be scored during movement (e.g., an } \\
\text { arm held stiffly while pacing) or in stationary } \\
\text { positions if it results in an abnormal body posture } \\
\text { (e.g., legs fully extended while sitting) } \\
\text { 4. Passive rubbing: touching, resting, or holding an } \\
\text { affected joint or body part on another body part for } \\
\text { at least } 3 \text { consecutive seconds } \\
\text { 5. Active rubbing: Massaging an affected joint or } \\
\text { body part for at least } 3 \text { consecutive seconds. } \\
\text { Massaging may be performed against another body } \\
\text { part of an object }\end{array}$ \\
\hline
\end{tabular}




$\begin{array}{ll}\text { Keefe et } & 87 \text { patients with } \\ \text { al., 1987 } & \text { osteoarthritis; } \\ & \text { coding of } \\ \text { standardized } \\ \text { movements }\end{array}$

Jensen et al., 1989

Prkachin et al., 2002

FuchsLacelle et al., 2003
61 females (mean age $=41$ years) with non-chronic back pain; coding of video of standardized maneuvers examination
1. Guarding: as Keefe and Block, 1982

2. Active rubbing of the knee: hands moving over or grabbing the affected knee(s) and the legs, palms down

3. Rigidity: as Anderson et al., 1987

4. Unloading the joints: shifting weight from one leg to the other while standing

5. Joint flexion: flexing of the affected knee(s) while in a static position (i.e., during standing or sitting)

1. Guarding: abnormally stiff, interrupted or rigid movement during shifting or pacing. Including use of canes or walkers, but cannot occur during a stationary position. Movement must be hesitant or interrupted rather than merely slow.

2. Bracing: position in which an almost fully extended limb supports and maintains an abnormal distribution of weight. It cannot occur during movement, must be held for at least 3 consecutive seconds. Most frequently is the gripping of edge of a table, cane or walker while sitting or standing. Bracing can occur with a leg if the subject leans against a wall using no other support, but it is not simply the shifting of weight when standing.

3. Passive rubbing: as Anderson et al., 1987

4. Active rubbing: as Anderson et al., 1987

1. Guarding: behaviour that prevents or alleviates pain, including stiffness, hesitation, limping, bracing, and flinching (adapted from Keefe \& Block, 1982)

2. Touching/rubbing: any contact between the patient's hands and the lower back, the hips, buttock, and the outer aspect of the thighs physical

93 older patients (mean age $=73$ years) post-knee surgery; coding of video of postsurgery physiotherapy
1. Guarding: as Keefe and Block, 1982

2. Bracing: as Keefe and Block, 1982

3. Rubbing: as Keefe and Block, 1982

4. Active Rubbing: as Keefe et al., 1987

5. Unloading joint: as Keefe et al., 1987

6. Rigidity: as Anderson et al., 1987

7. Joint-flexing: as Keefe et al., 1987 
2003). Thus, it should not be assumed that individuals experiencing similar pain intensity would display the same pain-related body movements, nor would the same pain-related body movements equal same pain intensity (Waters, Riordan, Keefe, \& Lefebvre, 2008); which highlights the importance of an instrument which is sensitive to a range of possible pain expressions.

Given that body movements associated with pain may be operationalized and reliably measured in older individuals with pain, it may be important to determine whether such body movements are displayed by LTC residents with dementia and observed by LTC staff (Blomqvist \& Hallberg, 2001; Closs, Cash, Barr, \& Briggs, 2005; Kovach, Griffie, Muchka, Noonan, \& Weissman, 2000). Studies in which older adults with dementia were observed by nursing staff for body movements related to pain indicate convergence between behaviours observed naturalistically and those coded from video recordings. For instance, Shega and colleagues (2008) applied the PBM with LTC residents and found that all pain-related body movements were more frequently observed in residents with chronic low back pain. Interestingly, the researchers also found that some body movements were more prevalent in residents with dementia than those who were cognitively intact (i.e., guarding, rubbing), which leads to the question of whether certain body movements may also be due to neurological issues associated with dementia (Shega et al., 2008). As yet, there are no published studies differentiating the cause of body movements in dementia as resulting from pain versus other neurodegenerative processes (i.e., badykinesia, parkinsonian signs). The complex presentations of persons with dementia highlights the difficulty of diagnosing pain in this group, thus comparison between baseline and subsequent scores may be more helpful than interpreting PBM 
scores alone. As well, based on consensus among LTC nurses, a number of pain-related body movements for residents with severe dementia were reported: restlessness, tense muscles, pulling away when touched, changes in mobility and gait, rubbing or holding body part (Kovach et al., 2000). Grabbing onto objects for help, lying down, sighing or moaning, stooping, and struggling were also found to be indicative of pain based on LTC resident and nurse consensus (Weiner et al., 1999c). Moreover, when intervened with pain medication, pain-related body movements (e.g., rigidity, knees pulled up) and other nonverbal indicators of pain were found to decrease (Warden et al., 2003). Together, systematic study of pain-related body movements, as well as nurse and LTC resident reports of pain-related body movements, inform the revisions of the PACSLAC.

1.6.4 Mental status changes and overlap with delirium. Assessment of changes in an older adult's mental status was also recommended as a possible indicator of pain (AGS Panel on Persistent Pain in Older Persons, 2002). However, mental status changes are not specific to pain, and may indicate another common condition in older adults with dementia, known as delirium (Fick, Agostini, \& Inouye, 2002; Laurila, Pitkala, Strandberg, \& Tilvis, 2004). According to the DSM-IV-TR (American Psychiatric Association, 2000), diagnosis of delirium involves a disturbance of consciousness with a change in cognition that develops over hours to days, and fluctuates over the course of the day. Specifically, the alteration in consciousness is presented as reduction in the ability to focus, sustain or shift attention, leading the individual to wander, perseverate, and distract easily. The change in cognition may be presented as memory impairment, disorientation, speech or perceptual disturbances. In addition, disruption in the sleepwake cycle, psychomotor agitation and flatness, emotional changes such as anxiety, fear, 
depression, irritability, anger, euphoria or apathy, are also associated with delirium. Disturbance in psychomotor activity and emotion can present as picking at bedclothes, attempting to get out of bed, or sudden aggressive behaviours. Finally, the symptoms associated with delirium tend to be caused by a medical condition which can be found upon history taking, physical exam or laboratory tests.

As apparent from the similarity of their presentations, delirium and pain in older adults with dementia are difficult to differentiate. Adding to the difficulty is the high comorbidity between delirium and dementia, where the prevalence of delirium in those with dementia has been found to be 22 to $89 \%$, with higher prevalence in hospitalized older adults with dementia (Fick et al., 2002). In terms of underlying causes, delirium and pain also overlap, where delirium may be caused by physiological problems such as overmedication or an acute medical condition, and has been associated with urinary tract infection, surgery, stress and bereavement, and severe pain (Lerner, Hedera, Koss, Stucky, \& Friedland, 1997). Delirium is also associated with increased mortality in older adults. Hospitalized older patients showed a 20 to $75 \%$ chance of mortality if delirium develops during hospitalization (Fick et al., 2002). Thus, it is imperative that both pain and delirium be accurately assessed in older adults with dementia. Misinterpretation of symptoms could lead to mistreatment of pain or delirium, with serious consequences.

As mentioned, Lints-Martindale et al. (2012) investigated the effect of removing delirium-related items from the PACSLAC. Removal of items was determined by selecting out PACSLAC items that overlapped in construct with delirium, based on the DSM-IV-TR (American Psychiatric Association, 2000) diagnostic criteria, the Confusion Assessment Method (CAM; Inouye , 2003; Inouye et al., 1990), and expert consensus 
between a geriatric psychiatrist and psychologist specialized in geropsychology (LintsMartindale et al., 2012). In particular, the CAM is an established tool for identifying delirium, and consists of nine criteria:

1. Acute onset of mental status change

2. Inattention (e.g., difficulty focusing on what is being said)

3. Disorganized thinking (e.g., rambling and incoherent speech)

4. Altered level of consciousness (e.g., coma or not able to be roused)

5. Disorientation (e.g., confusion about current location)

6. Memory impairment (e.g., difficulty in remembering instructions)

7. Perceptual disturbances (e.g., hallucinations)

8. Psychomotor agitation (e.g., picking at bedclothes)

9. Psychomotor retardation (e.g., staring into space and moving slowly)

For example, while agitation, verbal and physical aggression have been associated with pain in residents with dementia (Buffum, Miaskowski, Sands, \& Brod, 2001; M. Lemay \& Landreville, 2010), these behaviours are also symptomatic of delirium. The aim of eliminating these items was to prevent the artificial inflation of pain score as well as the misdiagnosis of pain. Lints-Martindale (2012) found that removal of delirium-related items did not affect PACSLAC discriminant validity, and effect sizes for discriminating between pain and baseline scores prior to and after item removal were relatively unchanged (i.e., change in effect size of 0.01 for needle injection condition, 0.04 for movement condition).

Though acute changes in a resident's mental status likely signals an important clinical event requiring examination, assessment of pain based on this criterion poses the 
challenge of symptom overlap, as well as requiring knowledge about the resident's baseline behaviours. It has been suggested that AGS behavioural domains may differ in their ability to signal pain to an observer, where facial expressions, vocalizations, and body movements may be most helpful for identifying pain based on observation alone. As mentioned, facial expressions, vocalizations and body movements may be identified without personal knowledge of the resident, though knowledge about the resident's baseline would greatly aid in pain assessment (Lints-Martindale et al., 2012). Thus, revisions to the PACSLAC involved an under-emphasis of items related to mental status changes and those that overlap with delirium. Nonetheless, in order to cover all of the AGS (2002) recommended domains for pain assessment, one item was designed to address mental status changes.

1.6.5 Changes in interpersonal interactions and activity patterns. Compared to other domains recommended for pain assessment, changes in interpersonal interactions and changes in activity patterns and routines have received less research attention. While changes in an older adult's interpersonal interactions and activity patterns may be due to pain, they may also be caused by other problems common in LTC residents, such as delirium, distress or other illnesses. Thus, changes from resident baseline behaviour may not be specific to pain but do serve as an important indicator for nurses and care aides to consider.

Similar to current knowledge on vocalizations associated with pain, information on pain-related changes in interpersonal interactions and activity patterns is largely descriptive and correlational in nature. However, a review of relevant findings was conducted in order to determine the extent to which PACSLAC items address these 
concerns. When 277 LTC residents with acute (i.e., due to injury) and chronic pain were observed, some common behaviours emerged (Cipher et al., 2006). For both residents with and without dementia, the most frequently observed dysfunctional behaviours accompanying acute and chronic pain were decreased activity level, weight loss and decreased appetite. Uniquely for residents with severe dementia in acute pain, physical combativeness and unsafe impulsive behaviours were more frequently observed. When experiencing chronic pain, residents with severe dementia also tended to show agitation, repetitive behaviours, delusional territorial behaviours, socially disruptive behaviours, wandering, as well as physical combativeness (Cipher et al., 2006). However, these behaviours are not unique to pain and may be comorbid with other medical problems (i.e., delirium and pain). Thus, the individual observing for pain indicators should be aware of the ambiguity of changes in interpersonal interactions and activity patterns in terms of underlying cause, and that while including behavioural changes to pain assessment may increase sensitivity to pain identification, corroboration with other behavioural domains (i.e., facial expressions, body movements) and reference to the resident's history is necessary. Thus, revision of PACSLAC items in the area of changes in interpersonal interactions and activity patterns also involved a de-emphasis of items not specific to pain, while retaining coverage of the AGS (2002) recommended domains for pain assessment (Lints-Martindale et al., 2012).

1.6.6 Physiological indicators. A behavioural domain that is not included in the recommendations by the AGS Panel is physiological indicators of pain, such as sweating, flushed complexion, laboured breathing. Indeed, reliance on physiological indicators has been criticized and it has been recommended that clinicians de-emphasize the use of 
physiological indicators for pain assessment as they do not discriminate between pain and other distress states, nor are they necessarily present when a person is in pain (Herr, Coyne, et al., 2006). Particularly for older adults with dementia, there is evidence that physiological responses, such as heart rate, respiratory rate and sympathetic skin response, may be blunted in response to pain (Mylius, Kunz, Hennighausen, Lautenbacher, \& Schepelmann, 2008; Porter et al., 1996). However, autonomic changes, such as pallor, alternated breathing, hypertension, continue to be cited as possible indicators of pain in older people (Collett, O’Mahoney, Schofield, Closs, \& Potter, 2007). As well, many observational pain tools include physiological indicators, and nurses and care-aides report paying attention to physiological indicators while assessing pain. When Lints-Martindale (2010) examined item endorsement in various observational pain tools (i.e., in PACSLAC, ADD, CNPI, NOPPAIN, PADE, PAINAD) by trained observers of patient videos of needle-injection and movement pain, it was found that certain physiological indicators were observed. The PACSLAC's physiological indicators items include “pale face”, “flushed, red face”, “teary eyed”, “sweating”, “shaking/trembling”, "cold and clammy." Of these, only "shaking/trembling” was endorsed in both needleinjection and movement pain (at $18 \%$ and $42 \%$ frequency, respectively). The PADE includes the items "loud/gasping breathing”, “rapid breathing/hyperventilating”, which were both endorsed in the pain states. "Loud/gasping breathing" was rated more frequently (11\% in needle-injection pain; 9\% in movement pain) compared to "rapid breathing/hyperventilating”, which was rated only $1 \%$ of the time in both pain states. The PAINAD also includes items on breathing, where “occasional laboured breathing” was endorsed more frequently ( $8 \%$ in needle-injection pain; $10 \%$ in movement pain) than 
“noisy laboured breathing” ( $0 \%$ in needle-injection pain; $1 \%$ in movement pain). It is uncertain whether these items were endorsed more frequently in pain states versus baseline condition, though this was likely difficult to examine due to the small number of participants for whom the physiological indicator was endorsed (i.e., small sample size).

These findings indicate that more research may be required before physiological indicators are struck from observational pain assessments. Thus, revision of the PACSLAC reflected some use of physiological indicators, with a focus on those that have been found to occur more frequently in research.

\subsection{Revisions to the PACSLAC}

According to Smith and McCarthy (1995), test refinement involves a set of procedures designed to improve how well a test measures a particular construct. As well, test refinement should occur when the constructs measured have become better understood and that more reliable measurement is possible (Silverstein \& Nelson, 2000). In the case of observational pain assessment in older adults with dementia, recent research on behavioural indicators of pain suggests that increased reliability in pain identification would be possible by considering these empirical findings (e.g., facial expressions, body movements, overlap with delirium).

In addition, Silverstein and Nelson (2000) suggested that test revision provides an opportunity to re-examine the constructs being measured. For instance, revision of the PACSLAC involved the consideration of symptom presentation overlap for pain and delirium, and that some PACSLAC items may represent both constructs, thus inflating pain scores. Thus, in attempting to improve content validity, test refinement also involves establishing the internal consistency and content homogeneity of the unidimensional 
facets of the construct measured (Smith \& McCarthy, 1995). Therefore, revisions to the PACSLAC were conducted as follows:

1. Organization of original PACSLAC items into AGS behavioural domains (i.e., facial expressions, vocalizations or verbalizations, body movements, changes in activity patterns and routine, changes in interpersonal interactions, mental status changes; Figure 1.1)

2. Elimination of items that overlapped with symptoms of delirium (LintsMartindale et al., 2012; Figure 1.2).

3. Revision of items, including the rewording, addition and deletion of items, based on the literature review (Figure 1.3).

The PACSLAC-II can be found in Appendix A. Following the development of PACSLAC-II, the present study was conducted in the two parts.

\section{Method and Results}

\subsection{Study 1: Quantitative Comparison of the PACSLAC and PACSLAC-II}

2.1.1 Purpose. The purpose of the first part of the study was to compare the PACSLAC and PACSLAC-II with respect to psychometric properties, and to assess any incremental validity of the revised tool.

2.1.2 Resident videos. Archival video footage of LTC residents with dementia were used in Study 1. Video segments were previously recorded as part of a study comparing observational pain tools for older adults with dementia (Lints-Martindale et al., 2012). Video recordings were taken on Sony HDRSR12 digital video cameras and edited using Windows Movie Maker for Windows XP. Each participant was filmed under 
Figure 1.1 PACSLAC items arranged by AGS domains

Facial Expressions

1. Grimacing

2. Sad look

3. Tighter face

4. Dirty look

5. Change in eyes (squinting, dull, bright, increased movement)

6. Frowning

7. Pain expression

8. Grim face

9. Clenching teeth

10. Wincing

11. Opening mouth

12. Creasing forehead

13. Screwing up nose

Verbalizations and Vocalizations

14. Screaming/yelling

15. Calling out (i.e., for help)

16. Crying

17. A specific sound or vocalization for pain, 'ow', 'ouch'

18. Moaning and groaning

19. Mumbling

20. Grunting

\section{Body Movements}

21. Fidgeting

22. Pulling away

23. Flinching

24. Restless

25. Pacing

26. Wandering

27. Trying to leave

28. Refusing to move

29. Thrashing

30. Moving slow

31. Impulsive behaviour (e.g., repetitive movements)

32. Uncooperative/resistant to care

33. Guarding sore area

34. Touching/holding sore area

35. Limping
36. Clenched fist

37. Going into foetal position

38. Stiff/rigid

39. Throwing things

Changes in Interpersonal Interactions

40. Physical aggression (e.g., pushing people and /or objects, scratching others, hitting others, striking, kicking)

41. Verbal aggression

42. Not wanting to be touched

43. Not allowing people near

44. Refusing medication

Changes in Activity Pattern and Routines

45. Changes in sleep: decreased

sleep or increased sleep during the day

46. Changes in appetite: decreased appetite or increased appetite

47. Decreased activity

\section{Mental Status Changes}

48. Agitated

49. Increased confusion

50. Angry/mad

51. Anxious

52. Upset

53. Cranky/irritable

54. Frustrated

\section{Other/Physiological Indicators}

\section{Pale face}

56. Flushed, red face

57. Teary eyed

58. Sweating

59. Shaking/trembling

60. Cold and clammy 
Figure 1.2 Elimination of PACSLAC items overlapping with delirium

\section{Facial expressions}

1. Grimacing

2. Sad look

3. Tighter face

4. Dirty look

5. Change in eyes (squinting, dull, bright, increased movement)

6. Frowning

7. Pain expression

8. Grim face

9. Clenching teeth

10. Wincing

11. Opening mouth

12. Creasing forehead

13. Screwing up nose

\section{Verbalizations and vocalizations}

\section{Sereaming/yelling \\ 15. Calling out (i.e., for help)}

16. Crying

17. A specific sound or vocalization for pain, 'ow', 'ouch'

18. Moaning and groaning

19. Mumbling

20. Grunting

\section{Body movements}

21. Fidgeting

22. Pulling away

23. Flinching

24. Restless

25. Pacing

26. Wandering

27. Trying to leave

28. Refusing to move

29. Thrashing

30. Moving slow

31. Impulsive behaviour (e.g., repetitive movements)

32. Uncooperative/resistant to care

33. Guarding sore area

34. Touching/holding sore area

35. Limping
Facial expressions

1. Grimacing

2. Sad look

3. Tighter face

4. Dirty look

5. Change in eyes (squinting, dull, bright, increased movement)

6. Frowning

7. Pain expression

8. Grim face

9. Clenching teeth

10. Wincing

11. Opening mouth

12. Creasing forehead

13. Screwing up nose

\section{Verbalizations and vocalizations}

14. Crying

15. A specific sound or vocalization for pain, 'ow', 'ouch'

16. Moaning and groaning

17. Grunting
Body movements

18. Pulling away

19. Flinching

20. Refusing to move

21. Thrashing

22. Moving slow

23. Guarding sore area

24 . Touching/holding sore area

25. Limping

26. Clenched fist

27. Going into foetal position

28. Stiff/rigid 
36. Clenched fist

37. Going into foetal position

38. Stiff/rigid

39. Throwing things

Changes in interpersonal interactions

40. Physical aggression (e.g., pushing people and /or objects, seratching others, hitting others, striking, kicking)

41. Verbal aggression

42. Not wanting to be touched

43. Not allowing people near

Changes in activity patterns or routines

44. Refusing medication

45. Changes in sleep: decreased sleep or increased sleep during the day

46. Changes in appetite: decreased appetite or increased appetite

47. Decreased activity

\section{Mental status changes}

48. Agitated

49. Increased confusion

50. Angry/mad

51. Anxious

52. Upset

53. Cranky/irritable

54. Frustrated

\section{Other/Physiological indicators}

55. Pale face

56. Flushed, red face

57. Teary eyed

58. Sweating

59. Shaking/trembling

60. Cold and clammy
Changes in interpersonal interactions

$\rightarrow \quad 29$. Not wanting to be touched

30. Not allowing people near
Changes in activity patterns or routines

31. Changes in appetite: decreased appetite or increased appetite

$\rightarrow \quad$ 32. Decreased activity
Mental status changes

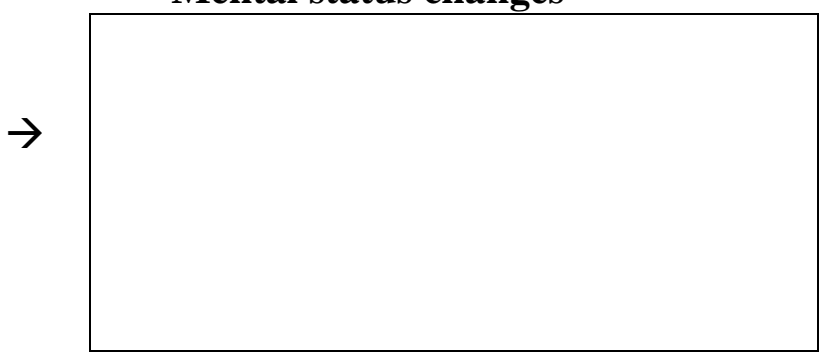

Other/Physiological indicators

33. Pale face

34. Flushed, red face

35. Teary eyed

36. Sweating

37. Shaking/trembling

38. Cold and clammy 
Figure 1.3 Revision of PACSLAC items (*these items were added or revised)

Facial expressions

1. Grimacing

2. Sadlook

3. Tighter face

4. Dirty look

5. Change in eyes (squinting, dull, bright, increased movement)

6. Frowning

7. Pain expression

8. Grim face

9. Clenching teeth

10. Wincing

11. Opening mouth

12. Creasing forehead

13. Serewing up nose
Facial expressions

1. Grimacing

2. Tighter face

3. Pain expression

4. Increased eye movement*

5. Wincing

6. Opening mouth

7. Creasing forehead

8. Lowered eyebrows or frowning*

9. Raised cheeks, narrowing of eyes or squinting*

10. Wrinkled nose and raised upper lip*

11. Eyes closing*

\section{Verbalizations and vocalizations}

14. Crying

15. A specific sound or vocalization for pain, 'ow', 'ouch'

16. Moaning and groaning
17. Grunting

Verbalizations and vocalizations

12. Crying

13. A specific sound for pain (e.g., 'ow', 'ouch')*

14. Moaning and groaning

15. Grunting

16. Gasping or breathing loudly*

Body movements

Body movements

18. Pulling away

19. Flinching

20. Refusing to move

21. Thrashing

22. Moving slow

23. Guarding sore area

24. Touching/holding sore area

25. Limping

26. Clenched fist

27. Going into foetal position

28. Stiff/rigid

Changes in interpersonal interactions

29. Not wanting to be touched

30. Not allowing people near
17. Flinching or pulling away*

18. Thrashing

19. Refusing to move

20. Moving slow

21. Guarding sore area

22. Rubbing or holding sore area*

23. Limping

24. Clenched fist

25. Going into foetal position

26. Stiff or rigid

27. Shaking or trembling*

Changes in interpersonal interactions

28. Not wanting to be touched

29. Not allowing people near 
Changes in activity patterns or routines

31. Changes in appetite: decreased appetite or increased appetite

32. Decreased activity

Mental status changes

\section{Other/Physiological indicators}

33. Pale face

34. Flushed, red face

35. Teary eyed

36. Sweating

37. Shaking/trembling

38. Cold and clammy
Changes in activity patterns or routines

30. Decreased activity

Mental status changes

31. Are there mental status changes that could be due to pain, and cannot be explained by another cause (e.g., delirium due to medication, etc.)?

\section{Other/Physiological Indicators}

32. Shaking/trembling*

(This item is moved to the Body

movements group) 
three conditions which were part of routine care for LTC residents in the Regina Qu'Appelle Health Region, and included (1) needle injection of the annual flu vaccine (i.e., acute pain), (2) activity during transfers, physiotherapy, range of motion exercises (i.e., movement-exacerbated pain), and (3) a baseline segment of the participant at rest prior to the needle injection or activity. After data collection, video segments were edited so that footage was similar in length. Needle injection footage was also edited in order to separate swabbing of the injection area and the injection itself, thus creating the swabbing condition. As such, participants who were filmed under all conditions appeared in a total of six video segments. The average length of the total video footage (i.e., all segments combined) was 2.52 minutes $(\mathrm{SD}=1.20)$ per participant. Residents in the video segments were identified only by a participant number and every effort was made to protect their privacy and dignity. That is, the videos were securely kept and access was given only to research personnel who adhered to the ethical principles and standards of the Canadian Psychological Association (2000).

2.1.3 Description of sample. Resident videos were recorded from a sample of 124 residents from 4 LTC facilities (Lints-Martindale et al., 2012). Recruitment of potential participants was assisted by the LTC facilities, who helped in identifying residents with a diagnosis of moderate to severe dementia and limited ability to communicate. Study information was sent out by the LTC facilities to proxies or legal guardians of the residents in order to obtain proxy consent. Resident assent was sought in all cases. When the resident expressed any verbal or behavioural indication that he or she was unwilling to participate in the study, he or she was not included regardless of proxy consent. Initially, 136 residents were recruited; however, 12 died prior to the completion of the study. Of the 
final 124 residents, 85 were filmed for both the needle-injection and movementexacerbated pain conditions. Fifteen residents were filmed only during needle-injection and 24 were filmed only during movement, resulting in a total of 100 residents filmed for needle-injection and 109 for movement-exacerbated pain. Reasons for not being filmed during either needle-injection or movement-exacerbated pain included death, resident refusal or withdrawal of consent, and lack of medical order for immunization.

In terms of sample characteristics, the sample of 124 residents consisted of 88 females (71\%) and 36 males (29\%). The average age of the sample was $83.94(S D=7.95$, range $=61-101)$. The average MMSE score of LTC residents was $5.35(S D=7.72$, range $=0-26$ ), indicating that on average the residents lived with a severe level of cognitive impairment. The average number of medical diagnoses for the LTC residents was 5.39 $(S D=2.28$, range $=1-12$; e.g., Alzheimer's disease, vascular dementia, osteoporosis, glaucoma, arthritis, etc.). Power calculations (i.e., setting alpha at 0.05 and power at 0.80 ) for repeated measures analyses suggest that the sample size available is more than adequate for the analyses.

2.1.4 Coding of videos. A trained research assistant blind to the hypotheses and goals of the study completed the PACSLAC-II after viewing the video segments. Each video segment was assigned a number and randomized for viewing. Immediately after each segment was viewed, the research assistant completed PACSLAC-II. A second coder also viewed and completed the PACSLAC-II for a randomly selected $20 \%$ of the video segments in order to establish inter-rater reliability. Data collected from ratings of videos by Lints-Martindale (2012) using the PACSLAC, PADE, PAINAD, ADD, CNPI, NOPPAIN were then compared to the newly-derived PACSLAC-II in statistical analyses. 
In addition, archival nurse rating of resident depression (i.e., CSDD; Cornell Scale for Depression in Dementia) were also considered in the analyses.

\subsubsection{Measures.}

2.1.5.1 Cornell Scale for Depression in Dementia (CSDD; Alexopoulos, Abrams, Young, \& Shamoian, 1988). The CSDD is a tool designed to assess depression in adults with mild to severe dementia. The 19-item tool is completed by the caregiver and requires the caregiver to rate symptoms of depression based on the week prior to the interview (i.e., a = unable to evaluate, $0=$ absent, $1=$ mild or intermittent, $2=$ severe). Five main symptom areas are assessed: mood-related signs, behavioural disturbance, physical signs, cyclic functions and cognitive disturbance. The CSDD has been found to have good interrater reliability $(\kappa=.67)$, internal consistency $(\alpha=.84)$, is sensitive to treatment and recommended for use with individuals with dementia (Alexopoulos et al., 1988; Conn \& Thorpe, 2007).

2.1.5.2 Assessment of Discomfort in Dementia (ADD; Kovach, Noonan, Griffie, Muchka, \& Weissman, 2001, 2002; Kovach, Weissman, Griffie, Matson, \& Muchka, 1999). The ADD is a tool designed for nurses to assess and treat pain and affective discomfort in individuals with dementia. The ADD was intended to be an intervention tool and include multiple steps which guide assessment and treatment response. Sections in the ADD lead nurses to (1) observe for pain indicators, (2) assess current and past history of pain, (3) assess environmental and interpersonal factors, (4) apply pharmacologic or nonpharmacologic intervention, and (5) consult with a physician. Only the pain assessment portion of this tool was examined in this study. The ADD covers all 6 behavioural

domains recommended by the AGS Persistent Pain Guidelines, including facial 
expression, mood, body language, voice and behaviour. Psychometric properties for the ADD have not been well-researched. Use of the ADD has led to decrease in discomfort and increase in pharmacologic and non-pharmacologic interventions (Kovach et al., 1999). Based on findings from Lints-Martindale and colleagues (2012), the ADD showed high inter-rater reliability $(\kappa=.85)$, acceptable internal consistency $(\alpha=.70)$ during needleinjection pain, and lower internal consistency $(\alpha=.60)$ during movement-exacerbated pain.

2.1.5.3 Checklist of Nonverbal Pain Indicators (CNPI; Feldt, 2000). The CNPI is a 6-item tool designed to assess the presence or absence of nonverbal vocalizations, facial grimaces/winces, bracing, restlessness, rubbing, and verbal vocal complaints at rest and during movement. The CNPI has been found to have low internal consistency $(\alpha=.54)$, though this may be related to the limited number of items (Feldt, 2000; Nygaard \& Jarland, 2006). Inter-rater reliability was found to be moderate to good ( $\kappa=.63$ to .82; Feldt, 2000), and test-retest reliability was also found to be moderate to good ( $\kappa=.43$ to .66; Nygaard \& Jarland, 2006) when the item rubbing was excluded. Construct validity for the CNPI has been demonstrated through a strong correlation between CNPI and visual analogue scale scores by the same rater, and moderate correlation between two different raters (Nygaard \& Jarland, 2006). Based on findings from Lints-Martindale and colleagues (2012), the CNPI showed high inter-rater reliability $(\kappa=.93)$ and lower level of internal consistency ( $\alpha=.51$ and .47) during needle injection and movement-exacerbated pain.

2.1.5.4 Pain Assessment for the Dementing Elderly scale (PADE; Villanueva, Smith, Erickson, Lee, \& Singer, 2003). The PADE is a 24-item scale designed to assess 3 areas: (1) observable pain indicators, (2) rating of pain intensity, and (3) chart review of 
the resident's activities of daily living. Items are responded to either by likert scale or multiple choice. As the observational indicators of pain are contained in Part 1 of the scale (e.g., facial expressions, breathing patterns, postures), only this part is used in the present study. Part 1 of the PADE assesses 5 of the 6 behavioural domains of pain indicators recommended by the AGS Panel. In terms of psychometric properties, part 1 demonstrated acceptable to good internal consistency ( $\alpha=.77$ to .88 ), strong inter-rater reliability ( $r=$ .93 to .95$)$, and strong test-retest reliability $(r=.70)$. Completion time for the PADE is longer compared to other scales, due to complexity of item and response style. Based on findings from Lints-Martindale and colleagues (2012), part 1 of the PADE showed substantial inter-rater reliability $(\kappa=.71)$ and lower levels of internal consistency $(\alpha=.13$ and .27) during needle injection and movement-exacerbated pain.

2.1.5.5 Pain Assessment in Advanced Dementia Scale (PAINAD; Warden et al., 2003). The Pain Assessment in Advanced Dementia Scale is a 5-item scale designed to measure breathing, negative vocalizations, facial expression, body language and consolability in individuals with advanced dementia. Observers rate each behaviour on the scale of 0 to 2, where 0 represents normal functioning and 2 represents behaviours hypothesized to indicate pain (e.g., noisy labored breathing, loud moaning or groaning, facial grimacing, rigid, or unable to console). While raters have indicated that facial expressions, negative vocalization, and body language are valuable in measuring pain, breathing and consolability have received less agreement (van Iersel, Timmerman, \& Mullie, 2006). The PAINAD is currently one of the most researched observational pain tools for individuals with dementia and has been found to have good psychometric properties. In terms of administration, the PAINAD has shown strong clinical utility and 
may be completed within a few minutes. The PAINAD has demonstrated good internal consistency ( $\alpha=.69$ to .85 ), strong inter-rater reliability ( $r=.75$ to .97 ), and strong testretest reliability ( $r=.88$ to .90; Costardi et al., 2007; DeWaters et al., 2008; Schuler et al., 2007; Zwakhalen et al., 2006a). In terms of concurrent validity, the PAINAD has been shown to correlate well with observer pain intensity ratings and other observational pain tools ( $r=.81$ to .85; Zwakhalen et al., 2006a). For discriminant validity, the PAINAD correlated poorly with the CSDD (Leong, Chong, \& Gibson, 2006) and was able to differentiate between pain and non-pain states (Lints-Martindale et al., 2012). Based on findings from Lints-Martindale and colleagues (2012), the PAINAD showed high interrater reliability $(\kappa=.87)$ and acceptable internal consistency $(\alpha=.78$ and .73$)$ during needle injection and movement-exacerbated pain.

\subsubsection{Non-Communicative Patient's Pain Assessment Instrument (NOPPAIN;}

Snow et al., 2004). The NOPPAIN is a pain screening tool designed for nursing assistants to observe and assess for pain in residents with dementia. The tool consists of 4 main parts: (1) observing during care activities, (2) noting of pain responses, (3) noting of pain sites, (4) rating resident pain intensity. In part 1, the rater notes the presence or absence of pain in 9 care activities (e.g., turned resident in bed, dressed resident, bathed resident). In part 2, the rater notes the presence or absence of specific pain indicators and rate the intensity of the observed pain response on a scale of 0 to 5 (i.e., pain words, pain faces, bracing, pain noises, rubbing, and restlessness). Part 3 involves marking the location of pain and skin problems in residents. Part 4 involves the rating the resident's pain on a verbal descriptor scale. The NOPPAIN covers 3 of the 6 AGS recommended behavioural domains. In terms of psychometric properties, the internal consistency of the NOPPAIN is 
not well-researched. Inter-rater reliability was found to be moderate to strong, and intrarater consistency over time was also found to be good to very good (Horgas, Nichols, Schapson, \& Vietes, 2007). Validity of the NOPPAIN is supported via correlation of NOPPAIN scores and coding of pain behaviours from videos (Snow et al., 2004). The NOPPAIN also requires caregivers less than 1 minute to complete once care activities were conducted prior to using the tool (Horgas et al., 2007). Based on findings from LintsMartindale and colleagues (2012), substantial inter-rater reliability was found $(\kappa=.73)$, but lower internal consistency was found ( $\alpha=.41$ to .48) during both movementexacerbated and needle injection pain conditions.

2.1.6 Hypothesis. It was hypothesized that the PACSLAC-II would demonstrate (1) improved reliability and validity compared to the PACSLAC, (2) a positive correlation with other pain tools, (3) a lack of correlation with a non-pain related measure (i.e., CSDD), and (4) an ability to discriminate between pain and baseline conditions while controlling for the contribution of other measures (i.e., , PACSLAC, PADE, PAINAD, ADD, CNPI, NOPPAIN).

\subsubsection{Analyses and Results}

2.1.7.1 Reliability. All quantitative analyses were performed using PASW Statistics 18. Reliability of PACSLAC-II scores was calculated. Internal consistency of PACSLAC-II scores in each condition were calculated using Cronbach's alpha. Results show that internal consistencies in the vaccination and movement-exacerbated pain conditions were satisfactory ( $\alpha=.77$ vaccination, $\alpha=.74$ movement; Bland \& Altman, 1997), while internal consistency during the swabbing condition was lower $(\alpha=.56)$,

given that this was a control condition with no pain expressions expected. Due to the low 
variance in scores during baseline conditions, internal consistency was not calculated for the baseline conditions. Internal consistencies of certain PACSLAC-II item domains (i.e., facial expressions, verbalizations or vocalizations, body movements) were also examined, although these are not intended for independent scoring. As the last 3 domains in the PACSLAC-II (i.e., changes in interpersonal interactions, changes in activity patterns or routines and mental status changes) consist of 1 or 2 items, reliability statistics were not calculated for these domains. Internal consistencies are presented in Table 2.1. In order to assess inter-rater reliability, Cohen's Kappa was calculated. The PACSLAC-II demonstrated satisfactory inter-rater reliability $(\kappa=.63$; Altman, 1991).

2.1.7.2 Validity. Convergent and discriminant validity of the PACSLAC-II were determined by examining the relationship between PACSLAC-II scores and the scores of other observational pain assessment tools (e.g., PAINAD) and a non-pain related tool (e.g., CSDD). Pearson’s $r$ correlations are presented in Table 2.2.

2.1.7.3 Convergent validity. As expected (see Table 2.2), PACSLAC-II scores in the influenza vaccination and movement-exacerbated pain conditions are correlated with scores from other observational pain assessment tools. PACSLAC-II scores showed the strongest correlation with PACSLAC scores in the vaccination and movement-exacerbated pain conditions $(r(98)=.89, p<.01$ influenza vaccination; $r(108)=.81, p<.01$ movement), and the strongest correlation with NOPPAIN in the swabbing condition ( $r(93)$ $=.73, p<.01$ swabbing).

2.1.7.4 Discriminant validity. As expected (see Table 2.2), PACSLAC-II scores in the influenza vaccination and movement-exacerbated pain conditions are not correlated with scores from the Cornell Scale for Depression in Dementia (CSDD). 
Table 2.1 Internal consistency of PACSLAC-II scores.

\begin{tabular}{lccc}
\hline & $\begin{array}{c}\text { Swabbing } \\
\text { Condition } \\
(n=93)\end{array}$ & $\begin{array}{c}\text { Influenza } \\
\text { vaccination } \\
\text { Condition } \\
(n=98)\end{array}$ & $\begin{array}{c}\text { Movement- } \\
\text { exacerbated pain } \\
\text { Condition }\end{array}$ \\
& .56 & .77 & $.707)$ \\
\hline PACSLAC-II all items & .36 & .61 & .54 \\
Facial expressions & .19 & .41 & .25 \\
Verbalizations and & .42 & .54 & .48 \\
verbalizations & .42 & & .74 \\
\hline
\end{tabular}


Table 2.2 Correlations between PACSLAC-II and other measures for the swabbing, influenza vaccination, and movement-exacerbated pain conditions

\begin{tabular}{|c|c|c|c|}
\hline Measure & $\begin{array}{c}r \\
\text { for swabbing } \\
\text { condition } \\
(n=93)\end{array}$ & $\begin{array}{c}r \\
\text { for vaccination } \\
\text { condition } \\
(n=98)\end{array}$ & $\begin{array}{l}r \\
\text { for movement- } \\
\text { exacerbated } \\
\text { pain } \\
\text { condition } \\
(n=108)\end{array}$ \\
\hline 1. PACSLAC & $.66^{\mathrm{a}}$ & $.89^{\mathrm{a}}$ & $.81^{\mathrm{a}}$ \\
\hline 2. ADD & $.55^{\mathrm{a}}$ & $.74^{\mathrm{a}}$ & $.72^{\mathrm{a}}$ \\
\hline 3. CNPI & $.56^{\mathrm{a}}$ & $.78^{\mathrm{a}}$ & $.68^{\mathrm{a}}$ \\
\hline 4. NOPPAIN & $.73^{\mathrm{a}}$ & $.82^{\mathrm{a}}$ & $.81^{\mathrm{a}}$ \\
\hline 5. PADE & $.65^{\mathrm{a}}$ & $.77^{\mathrm{a}}$ & $.80^{\mathrm{a}}$ \\
\hline 6. PAINAD & $.68^{\mathrm{a}}$ & $.86^{\mathrm{a}}$ & $.79^{\mathrm{a}}$ \\
\hline 7. CSDD & -.05 &. .10 & -.06 \\
\hline $\begin{array}{l}\text { 8. PACSLAC-II } \\
\text { swabbing }\end{array}$ & - & $.40^{\mathrm{a}}$ & $.27^{\mathrm{a}}$ \\
\hline $\begin{array}{l}\text { 9. PACSLAC-II } \\
\text { vaccination }\end{array}$ & - & - & $.35^{\mathrm{a}}$ \\
\hline $\begin{array}{l}\text { 10. PACSLAC-II } \\
\text { movement-exacerbated } \\
\text { pain }\end{array}$ & - & - & - \\
\hline \multicolumn{4}{|c|}{$\begin{array}{l}\mathrm{a}=p<0.01 \\
\text { Note. PACSLAC = Pain Assessment Checklist for Seniors with Limited Ability to } \\
\text { Communicate; ADD = Assessment of Discomfort in Dementia; CNPI = Checklist of } \\
\text { Nonverbal Pain Indicators; NOPPAIN = Non-Communicative Patient's Pain Assessment } \\
\text { Instrument; PADE = Pain Assessment in Advanced Dementia Scale; PAINAD = Pain } \\
\text { Assessment in Advanced Dementia; CSDD = Cornell Scale for Depression Dementia. }\end{array}$} \\
\hline
\end{tabular}


2.1.7.5 Ability to discriminate between pain and baseline conditions. The ability of the PACSLAC-II to discriminate between baseline and pain states was tested by conducting repeated measures analyses of variance (ANOVA). Two within-subject repeated measures ANOVAs were conducted in order to compare the pain segment scores versus non-pain segment scores: a) vaccination versus swabbing versus baseline scores: b) the movement-exacerbated pain versus baseline score. For each ANOVA, PACSLAC-II scores were entered as the dependent variable. In order to account for the length of time of video segments, pairs of videos (e.g., influenza vaccination and its baseline, movementexacerbated pain and its baseline) were edited such that they were equivalent in length. Means and standard deviations during the swabbing period, influenza vaccination, movement-exacerbated pain, and baseline conditions are presented in Table 2.3. Results of the ANOVAs indicate that the PACSLAC-II was able to discriminate between baseline and pain in both conditions, for the vaccination condition, $F(2,92)=80.92, p<.001$, partial $\eta^{2}=.64$; and for the movement-exacerbated pain condition $F(1,105)=118.02, p<$ .001, partial $\eta^{2}=.53$. Paired comparisons showed that PACSLAC-II means for baseline, swabbing, vaccination differ significantly $p<.01$. Notably the magnitude of the effect was greatest for the vaccination condition, where the effect size was partial $\eta^{2}=.64$.

In addition, a within-subject repeated measures ANCOVA was conducted in order to determine whether the PACSLAC-II can discriminate across conditions (baseline vs. swabbing vs. vaccination vs. movement-exacerbated pain). In order to account for the different lengths of videos across conditions, the total viewing time was entered as a covariate. Results of the ANCOVA with time as a covariate were significant, $F(3,73)=$ 5.32, $p<.001$, partial $\eta^{2}=.07$; and post-hoc comparisons were conducted (Table 2.4). In 
Table 2.3 Means and standard deviations (SDs) of PACSLAC-II scores

\begin{tabular}{lll}
\hline Condition & Mean & $(S D)$ \\
\hline Baseline Swabbing $(n=96)$ & 1.17 & $(1.29)$ \\
Swabbing $(n=93)$ & 2.02 & $(1.86)$ \\
Baseline Influenza vaccination $(n=100)$ & 1.47 & $(1.41)$ \\
Influenza vaccination $(n=100)$ & 6.03 & $(3.78)$ \\
$\begin{array}{l}\text { Baseline Movement-exacerbated pain }(n= \\
\begin{array}{l}\text { 109) } \\
\text { Movement-exacerbated pain }(n=109)\end{array}\end{array}$ \\
\hline
\end{tabular}


Table 2.4 Paired comparisons of PACSLAC-II ANCOVA results with Bonferonni adjustment

\begin{tabular}{lccc}
\hline Pairwise comparison & Mean difference & SE & Sig. \\
\hline Baseline vs. swabbing & .84 & .23 & $p=.003$ \\
Baseline vs. vaccination & 5.03 & .43 & $p<.001$ \\
$\begin{array}{l}\text { Baseline vs. movement- } \\
\text { exacerbated pain }\end{array}$ & 4.99 & .40 & $p<.001$ \\
$\begin{array}{l}\text { Swabbing vs. vaccination } \\
\begin{array}{l}\text { Swabbing vs. movement- } \\
\text { exacerbated pain }\end{array}\end{array}$ & 4.18 & .41 & $p<.001$ \\
$\begin{array}{l}\text { Vaccination vs. movement- } \\
\text { exacerbated pain }\end{array}$ & .04 & .39 & $p<.001$ \\
& & .49 & n.s. \\
\end{tabular}


order to adjust for the increased probability of Type I error in multiple comparisons, a Bonferonni adjustment of $p<.008$ was used. Results showed that PACSLAC-II scores were significantly different across the baseline vs. swabbing conditions, baseline vs. pain conditions (i.e., vaccination and movement-exacerbated pain), and swabbing vs. pain conditions. Scores between vaccinations vs. movement-exacerbated pain conditions showed no significant difference.

In summary, results indicate that the PACSLAC-II is able to discriminate between baseline and pain condition (i.e., vaccination, movement-exacerbated pain). In order to test for potential differences between males and females, were repeated without male data in the sample and results were largely unchanged.

\subsubsection{Comparisons with other observational pain tools}

2.1.7.6.1 Effect size. In order to compare the PACSLAC-II’s ability to discriminate between pain and baseline to that of the other observational pain tools that were studied by Lints-Martindale et al. (2012), effect sizes (partial $\eta^{2}$ ) were examined (Table 2.5). Comparison of the partial eta squared shows that pain assessment tools (i.e., PACSLACII, PACSLAC, PADE, PAINAD, ADD, CNPI, and NOPPAIN) differ in terms of the amount variance accounted for by the tool. In the influenza vaccination vs. baseline comparison, the PACSLAC-II (partial $\eta^{2}=.63$ ) accounted for the most variance, followed by the PACSLAC (partial $\eta^{2}=.52$ ) and the PADE (partial $\eta^{2}=.52$ ). In the movementexacerbated pain vs. baseline comparison, PACSLAC-II (partial $\eta^{2}=.53$ ) also accounted for the most variance, followed by the PACSLAC (partial $\eta^{2}=.47$ ) and the NOPPAIN (partial $\eta^{2}=.38$ ). 
Table 2.5. Effect sizes (partial $\eta^{2}$ ) from ANOVAs comparing pain condition vs. baseline condition

\begin{tabular}{lcc}
\hline Measures & $\begin{array}{c}\text { Vaccination } \\
\text { Partial } \eta^{2}\end{array}$ & $\begin{array}{c}\text { Movement- } \\
\text { exacerbated pain } \\
\text { Partial } \eta^{2}\end{array}$ \\
\hline ADD & .45 & .28 \\
CNPI & .33 & .17 \\
NOPPAIN & .45 & .38 \\
PACSLAC & .52 & .47 \\
PACSLAC-II & .63 & .53 \\
PADE & .52 & .34 \\
PAINAD & .48 & .26
\end{tabular}

Note. Unbolded partial $\eta^{2}$ values taken from "A comparative investigation of observational pain assessment tools for older adults with dementia,” by A. C. Lints-Martindale, A. C., T. Hadjistavropoulos, L. M. Lix, \& L. Thorpe, 2012, Clinical Journal of Pain, 28, 226-237. PACSLAC = Pain Assessment Checklist for Seniors with Limited Ability to Communicate; ADD = Assessment of Discomfort in Dementia; CNPI = Checklist of Nonverbal Pain Indicators; NOPPAIN = Non-Communicative Patient's Pain Assessment Instrument; PADE = Pain Assessment in Advanced Dementia Scale; PAINAD = Pain Assessment in Advanced Dementia; CSDD = Cornell Scale for Depression Dementia. 
2.1.7.6.2 Controlling for contribution from other pain tools. Another method of examining a tool's unique ability to discriminate between pain and non-pain states was to conduct a series of analyses of covariance (ANCOVAs) while taking into account the contribution of other pain tools (Lints-Martindale et al., 2012). For each condition, the PACSLAC-II pain score (e.g., PACSLAC-II influenza vaccination score) was entered as the dependent variable, while difference scores of all other pain tools (i.e., PAINAD difference score $=$ PAINAD influenza vaccination score - PAINAD baseline score) were entered as covariates. Difference scores indicate the amount of variance measured by each assessment instrument from the baseline to the pain condition (Hededker \& Gibbons, 2006; Lix \& Fouladi, 2007). If the analysis becomes non-significant once the covariates are added, it would indicate that other pain tools together account for a greater part of the variance than the PACSLAC-II alone. However, if the analysis remains significant, this would indicate that the pain tool accounts for greater variance than all of the other pain tools combined. In terms of the covariates entered, the ANCOVA was conducted with the PACSLAC difference score and without the PACSLAC difference score entered in the covariate. This was done to allow for comparison of the PACSLAC II results (over and above the variance accounted for by the other measures) with the corresponding results on the PACSLAC’s ability to discriminate pain while accounting for

First, when difference scores of the ADD, CNPI, NOPPAIN, PAINAD and PADE were entered as covariates in the ANCOVA, it was found that the PACSLAC-II was able to differentiate between baseline and pain conditions during both the vaccination, $F(1,98)$ $=29.72, p<.001$, partial $\eta^{2}=.24$; and movement-exacerbated pain conditions, $F(1,100)$ $=26.75, p<.001$, partial $\eta^{2}=.21$. 
Second, PACSLAC difference scores were added to the covariates in the ANCOVA. Results indicate that the PACSLAC-II retained its ability to discriminate between pain and baseline. Specifically, the PACSLAC-II was able to differentiate between baseline and pain conditions during both the vaccination, $F(1,91)=24.41, p<$ .001 , partial $\eta^{2}=.21$; and movement-exacerbated pain condition, $F(1,99)=17.19, p<$ .001 , partial $\eta^{2}=.15$. Addition of PACSLAC difference scores led slight decreases in effect sizes, which is expected due to the similarities across the PACSLAC and PACSLAC-II and likely overlap in variance accounted for.

These analyses were also conducted with only data from female participants and differentiations between pain and baseline conditions with difference scores as covariates remained significant.

\subsubsection{Exploration of individual items and subscales}

2.1.7.7.1 Frequency of endorsement. The frequency that individual PACSLAC-II items were endorsed in each condition was examined (see Table 2.6). In addition, McNemar's tests were conducted in order to determine whether any items were more likely to be endorsed in the influenza vaccination condition versus the movementexacerbated pain condition. The McNemar's test was chosen as it is the test that is considered most appropriate for examining variables with dichotomous, categorical outcomes (i.e., $0=$ not observed; $1=$ present). The null hypothesis is that the proportion of observed behaviour in the vaccination condition is the same as the proportion of observed behaviour in the movement-exacerbated pain condition. Results of the McNemar's test showed that the items "grimacing," "wincing," “creasing forehead," "a specific sound for pain (e.g., ow, ouch)," "flinching or pulling away," and "trembling or shaking” were more 
Table 2.6 Percentage frequency of PACSLAC-II items coded during each condition

\begin{tabular}{|c|c|c|c|c|c|c|}
\hline PACSLAC-II items & $\begin{array}{l}\text { Baseline } \\
\text { Swabbing }\end{array}$ & Swabbing & $\begin{array}{c}\text { Baseline } \\
\text { Influenza } \\
\text { vaccination }\end{array}$ & $\begin{array}{c}\text { Influenza } \\
\text { vaccination }\end{array}$ & $\begin{array}{l}\text { Baseline } \\
\text { Move- } \\
\text { ment } \\
\text { exacer- } \\
\text { bated pain }\end{array}$ & $\begin{array}{c}\text { Movement } \\
\text { exacer- } \\
\text { bated pain }\end{array}$ \\
\hline 1. grimacing* & $0 \%$ & $2.4 \%$ & $0 \%$ & $26.5 \%$ & $1.9 \%$ & $11.1 \%$ \\
\hline 2. tighter face & $5.6 \%$ & $10.5 \%$ & $8.1 \%$ & $32.7 \%$ & $15.0 \%$ & $36.1 \%$ \\
\hline $\begin{array}{l}\text { 3. pain } \\
\text { expression }\end{array}$ & $.8 \%$ & $0 \%$ & $1.0 \%$ & $13.3 \%$ & $1.9 \%$ & $13.0 \%$ \\
\hline $\begin{array}{l}\text { 4. increased } \\
\text { eye }\end{array}$ & $0 \%$ & $.8 \%$ & $2.0 \%$ & $7.1 \%$ & $4.7 \%$ & $11.1 \%$ \\
\hline $\begin{array}{l}\text { movement } \\
\text { 5. wincing* }\end{array}$ & $0 \%$ & $1.6 \%$ & $1.0 \%$ & $26.5 \%$ & $0.9 \%$ & $9.3 \%$ \\
\hline $\begin{array}{l}\text { 6. opening } \\
\text { mouth }\end{array}$ & $11.3 \%$ & $17.7 \%$ & $28.3 \%$ & $58.2 \%$ & $30.8 \%$ & $60.2 \%$ \\
\hline $\begin{array}{l}\text { 7. creasing } \\
\text { forehead* }\end{array}$ & $14.5 \%$ & $16.9 \%$ & $28.3 \%$ & $37.8 \%$ & $31.8 \%$ & $62.0 \%$ \\
\hline $\begin{array}{l}\text { 8. lowered } \\
\text { eyebrows or } \\
\text { frowning }\end{array}$ & $22.6 \%$ & $28.2 \%$ & $28.3 \%$ & $62.2 \%$ & $40.2 \%$ & $56.5 \%$ \\
\hline 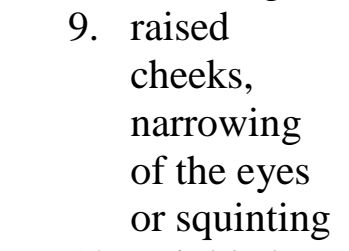 & $8.9 \%$ & $8.9 \%$ & $10.1 \%$ & $45.9 \%$ & $29.0 \%$ & $45.4 \%$ \\
\hline $\begin{array}{l}\text { 10. wrinkled } \\
\text { nose and } \\
\text { raised upper } \\
\text { lip }\end{array}$ & $3.2 \%$ & $7.3 \%$ & $6.1 \%$ & $35.7 \%$ & $10.3 \%$ & $29.6 \%$ \\
\hline 11. eyes closing & $3.2 \%$ & $5.6 \%$ & $8.1 \%$ & $32.7 \%$ & $14.0 \%$ & $26.9 \%$ \\
\hline 12. crying & $0 \%$ & $0 \%$ & $0 \%$ & $1.0 \%$ & $0 \%$ & $0.9 \%$ \\
\hline $\begin{array}{l}\text { 13. a specific } \\
\text { sound for } \\
\text { pain (e.g., } \\
\text { ow, ouch)* }\end{array}$ & $0 \%$ & $.8 \%$ & $1.0 \%$ & $24.5 \%$ & $1.9 \%$ & $8.3 \%$ \\
\hline $\begin{array}{l}\text { 14. moaning } \\
\text { and } \\
\text { groaning }\end{array}$ & $.8 \%$ & $4.0 \%$ & $1.0 \%$ & $21.4 \%$ & $7.5 \%$ & $19.4 \%$ \\
\hline 15. grunting & $1.6 \%$ & $.8 \%$ & $0 \%$ & $1.0 \%$ & $2.8 \%$ & $6.5 \%$ \\
\hline $\begin{array}{l}\text { 16. gasping or } \\
\text { breathing } \\
\text { loudly }\end{array}$ & $0 \%$ & $1.6 \%$ & $1.0 \%$ & $13.3 \%$ & $2.8 \%$ & $12.0 \%$ \\
\hline
\end{tabular}


17. flinching or $\quad 1.6 \% \quad 4.0 \% \quad 3.0 \% \quad 46.9 \% \quad 4.7 \% \quad 13.0 \%$ pulling away*

18. thrashing

$0 \% \quad 0 \%$

$0 \%$

$1.0 \%$

$0 \%$

$0 \%$

19. refusing to

$0 \%$

$.8 \%$

$0 \%$

$0 \%$

$0 \%$

$8.3 \%$

move

20. moving

$0 \%$

$0 \%$

$0 \%$

$0 \%$

$0.9 \%$

$13.0 \%$ slow

21. guarding

$0 \%$

$0 \%$

$0 \%$

$6.1 \%$

$0 \%$

$1.9 \%$ sore area

22. rubbing or

$0 \%$

$0 \%$

$0 \%$

$2.0 \%$

$0 \%$

$4.6 \%$

holding sore area

23. limping

$0 \%$

$0 \%$

$0 \%$

$0 \%$

$0.9 \%$

$9.3 \%$

24. clenched

$0 \%$

$4.0 \%$

$0 \%$

$11.2 \%$

$2.8 \%$

$19.4 \%$

fist

25. going into

$1.6 \%$

$0 \%$

$4.0 \%$

$6.1 \%$

$3.7 \%$

$6.5 \%$

foetal position

26. stiff or rigid

$6.5 \%$

$21.0 \%$

$6.1 \%$

$59.2 \%$

$15.0 \%$

$43.5 \%$

27. shaking or

$8.9 \%$

$12.1 \%$

$10.1 \%$

$25.5 \%$

$23.8 \%$

$57.4 \%$ trembling*

28. not wanting

$0 \%$

$2.4 \%$

$0 \%$

$4.1 \%$

$0.9 \%$

$3.7 \%$

to be touched

29. not

allowing people near

30. decreased activity

31. are there mental status changes due to pain

Note. ${ }^{*}$ Items that are more likely to be endorsed in one pain condition versus the other (i.e., vaccination vs. movement-exacerbated pain), $p<.05$. 
likely to be coded in one pain condition compared to another. Specifically, "grimacing" was endorsed more frequently in the vaccination condition $\left(n=83, \chi^{2}[1]=21.76, p<\right.$ .001). "Wincing" was also endorsed more frequently in the vaccination condition ( $n=83$, $\left.\chi^{2}[1]=.72, p=.001\right)$. "Creasing forehead" was endorsed more frequently in the movement-exacerbated pain condition $\left(n=83, \chi^{2}[1]=2.82, p=.001\right)$. "A specific sound for pain” was endorsed more frequently in the vaccination condition $\left(n=83, \chi^{2}[1]=2.37\right.$, $p=.003)$. "Flinching or pulling away" was endorsed more frequently in the vaccination condition ( $\left.n=83, \chi^{2}[1]=8.23, p<.001\right)$. "Shaking or trembling" was endorsed more frequently in the movement-exacerbated pain condition $\left(n=83, \chi^{2}[1]=4.12, p<.001\right)$.

2.1.7.7.2 Item-total correlation. To further explore individual items, item-total correlations were calculated to see to what degree each item related to the total score. Low values are thought to indicate that the item is assessing a construct that may be different from the scale as a whole. In the vaccination condition, item-total correlations ranged from $r=-.001$ ("thrashing") to .71 ("grimacing”). In the movement-exacerbated pain condition, item-total correlations ranged from $r=-.17$ ("limping”) to .56 ("grimacing”). Results are presented in Table 2.7.

2.1.7.7.3 Subscale ability to differentiate across conditions. Exploratory withinsubjects repeated measures ANCOVAs were conducted in order to determine whether the PACSLAC-II subscales (i.e., facial expressions, verbalizations and vocalizations, body movements) are able to discriminate across pain and non-pain conditions (baseline vs. swabbing vs. vaccination vs. movement-exacerbated pain). The last 3 subscales (i.e., changes in interpersonal interactions, changes in routine and activity patterns, mental status changes) were not subjected to the analyses due to low variance and endorsement of 
Table 2.7 Item-total correlations of PACSLAC-II scores.

\begin{tabular}{|c|c|c|}
\hline PACSLAC-II items & $\begin{array}{c}\text { Influenza } \\
\text { vaccination }\end{array}$ & $\begin{array}{c}\text { Movement- } \\
\text { exacerbated pain }\end{array}$ \\
\hline 1. grimacing & .71 & .56 \\
\hline 2. tighter face & -.25 & .24 \\
\hline 3. pain expression & .08 & .38 \\
\hline $\begin{array}{l}\text { 4. increased eye } \\
\text { movement }\end{array}$ & -.07 & .20 \\
\hline 5. wincing & .32 & 19 \\
\hline 6. opening mouth & .42 & .34 \\
\hline 7. creasing forehead & -.02 & .04 \\
\hline $\begin{array}{l}\text { 8. lowered eyebrows } \\
\text { or frowning }\end{array}$ & .46 & .29 \\
\hline $\begin{array}{l}\text { 9. raised cheeks, } \\
\text { narrowing of the } \\
\text { eyes or squinting }\end{array}$ & .51 & .22 \\
\hline $\begin{array}{l}\text { 10. wrinkled nose and } \\
\text { raised upper lip }\end{array}$ & .64 & 39 \\
\hline 11. eyes closing & .53 & .34 \\
\hline 12. crying & .16 & .14 \\
\hline $\begin{array}{l}\text { 13. a specific sound for } \\
\text { pain (e.g., ow, } \\
\text { ouch) }\end{array}$ & .63 & .31 \\
\hline $\begin{array}{l}\text { 14. moaning and } \\
\text { groaning }\end{array}$ & .60 & .47 \\
\hline 15. grunting & .05 & .20 \\
\hline $\begin{array}{l}\text { 16. gasping or } \\
\text { breathing loudly }\end{array}$ & .18 & .26 \\
\hline $\begin{array}{l}\text { 17. flinching or pulling } \\
\text { away }\end{array}$ & .54 & .48 \\
\hline 18. thrashing & -.001 & $*$ \\
\hline 19. refusing to move & * & .24 \\
\hline 20. moving slow & $*$ & .12 \\
\hline 21. guarding sore area & .34 & .04 \\
\hline $\begin{array}{l}\text { 22. rubbing or holding } \\
\text { sore area }\end{array}$ & .15 & .10 \\
\hline
\end{tabular}


23. limping

24. clenched fist

25. going into foetal position

26. stiff or rigid

27. shaking or trembling

28. not wanting to be touched

29. not allowing people near

30. decreased activity

31. are there mental status changes due to pain

$$
*
$$

.04

.15

.32

.39

.29

.16

$*$

$*$

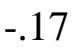

.38

.37

.44

.22

.38

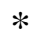

$*$

$*$

Note. ${ }^{*}$ item was not included in calculation of item-total statistic due to zero or approximately zero variance. 
items. In order to account for the different lengths of video segments across conditions, the total viewing time was entered as a covariate.

For the facial expressions subscale, results of the ANCOVA was significant, $F$ (3, $73)=5.27, p<.01$, partial $\eta^{2}=.07$. Post-hoc comparisons with a Bonferonni adjustment of $p<.008$ showed significant mean differences (Table 2.8) across the baseline vs. pain conditions (i.e., vaccination and movement-exacerbated pain), and swabbing vs. pain conditions. However, scores between the vaccinations vs. movement-exacerbated pain conditions, and baseline vs. swabbing conditions showed no significant difference.

For verbalizations and vocalizations, the ANCOVA was not significant, $F(3,73)$ $=1.93, p=.13$.

For body movements, the ANCOVA with time as a covariate was significant, $F$ (3, $71)=4.30, p=.006$, partial $\eta^{2}=.06$. As with the facial expressions subscale results, posthoc comparisons with a Bonferonni adjustment of $p<.008$ showed significant mean differences (Table 2.9) across the baseline vs. pain conditions (i.e., vaccination and movement-exacerbated pain), and swabbing vs. pain conditions. However, scores between the vaccinations vs. movement-exacerbated pain conditions, and baseline vs. swabbing conditions showed no significant differences.

\subsection{Study 2: Feedback from LTC Nurses and Care Aides}

2.2.1 Purpose. The purpose of the second part of this investigation was for LTC front line staff (e.g., nurses and care aides) to use the PACSLAC and PACSLAC-II in practice with residents with dementia, and to collect narrative feedback regarding relative clinical utility. 
Table 2.8. Paired comparisons of Facial Expression Subscale ANCOVA results with Bonferonni adjustment

\begin{tabular}{lccc}
\hline Pairwise comparison & Mean difference & SE & Sig. \\
\hline Baseline vs. swabbing & .43 & .15 & n.s. \\
Baseline vs. vaccination & 2.95 & .25 & $p<.001$ \\
$\begin{array}{l}\text { Baseline vs. movement- } \\
\text { exacerbated pain }\end{array}$ & 2.86 & .23 & $p<.001$ \\
$\begin{array}{l}\text { Swabbing vs. vaccination } \\
\begin{array}{l}\text { Swabbing vs. movement- } \\
\text { exacerbated pain }\end{array}\end{array}$ & 2.52 & .23 & $p<.001$ \\
$\begin{array}{l}\text { Vaccination vs. movement- } \\
\text { exacerbated pain }\end{array}$ & .09 & .24 & $p<.001$ \\
& & .31 & $n . s$. \\
\hline
\end{tabular}


Table 2.9 Paired comparisons of Body Movement Subscale ANCOVA results with Bonferonni adjustment

\begin{tabular}{lccc}
\hline Pairwise comparison & Mean difference & SE & Sig. \\
\hline Baseline vs. swabbing & .33 & .10 & n.s. \\
Baseline vs. vaccination & 1.37 & .16 & $p<.001$ \\
$\begin{array}{l}\text { Baseline vs. movement- } \\
\text { exacerbated pain }\end{array}$ & 1.51 & .17 & $p<.001$ \\
$\begin{array}{l}\text { Swabbing vs. vaccination } \\
\begin{array}{l}\text { Swabbing vs. movement- } \\
\text { exacerbated pain }\end{array}\end{array}$ & 1.04 & .14 & $p<.001$ \\
$\begin{array}{l}\text { Vaccination vs. movement- } \\
\text { exacerbated pain }\end{array}$ & .13 & .16 & $p<.001$ \\
& & .16 & n.s. \\
\hline
\end{tabular}


2.2.2 Participants. Twenty-six LTC staff members used the PACSLAC or the PACSLAC-II for a period of 4 to 6 weeks and provided feedback to the researcher in an individual interview. Of the 26 participants, 16 were nurses and 10 were care aides. The majority of the participants were female $(n=25)$. The average age of the sample was 47.62 years $(S D=9.63)$. Participants had worked within the long-term care field for 16.53 years $(S D=9.97)$. Of the 26 participants, 16 reported receiving previous education in pain management of varying lengths, whereas 10 reported receiving none.

2.2.3 Procedure. LTC nurses and care aides from rural health regions were invited to participate in a study evaluating an observational pain tool for residents with dementia. Recruitment of nurses and care aides was facilitated by LTC homes' directors of care, who informed eligible staff about the project while emphasizing the voluntary nature of participation. Eligibility criteria were that the nurse or care aide be working regularly (e.g., an average of 3 shifts per week) on a unit with residents with dementia and limited ability to communicate. At the initial meeting the researcher met with potential participants in person in order to obtain informed consent, and to explain study rationale, procedure anduse of the tools. Each staff participant was asked to complete the assessment checklist (either the PACSLAC or the PACSLAC-II) for up to 3 of their patients with dementia, 3 times each week over approximately a one-month period. Assignment to PACSLAC vs. PACSLAC-II conditions was determined by coin toss until the desired number of participants was reached for one of the tools. Subsequent to that, the remaining staff participants were assigned to the other tool. Out of 26 participants, 9 nurses and 5 care aides used the PACSLAC, while 7 nurses and 5 care aides used the PACSLAC-II. 
2.2.3.1 PACSLAC Instructions. Once staff members consented to participating in the study, they were given verbal and written instructions on the use of the PACSLAC or PACSLAC-II (Appendix B), as well as an opportunity to ask questions, prior to beginning its usage. Specifically, staff participants were be told the purpose of the tool (i.e., to screen for pain in older adults with dementia and limited ability to communicate), as well as when and how to use the tool. Participants were asked to use the tool either at the end of each shift, or consistently after specific moments of possible pain, especially moments involving movement of the body, such as transfers. Staff participants were also told that changes in the total score likely reflect an increase or decrease in pain. At the same time, it was explained that the tool is intended to be an individualized screening measure, such that there is no cut-off score and scores cannot be compared across individuals, and that resident's medical conditions and history should be considered.

Following the initial meeting with participants where consent was obtained, the researcher maintained contact with participants in order to address potential questions, via brief visits to the facility and/or brief phone calls to the participants. Each participant completed about a total of 15 to 36 checklists, which allowed the participant to observe the presentation of a range of behavioural indicators in individual patients and across patients. Participants also charted pain scores on a pain graph (Appendix C) provided along with the checklists, for observing change in pain score over time. Staff participants were asked to not include any patient information and so no patient information was collected for this study. Date and time of checklist administration and the checklist packages were collected in order to monitor compliance. 
2.2.3.2 Staff interviews. Once participants indicated that they had completed their checklists, a follow-up meeting was scheduled at the participant's facility in order to conduct a feedback interview. Prior to the interview, participants completed a demographic information form (Appendix D) and a ratings form on the clinical usefulness of the PACSLAC or PACSLAC-II (Appendix E). Interviews were about 20 to 30 minutes in length and followed a semi-structured format querying the benefits and challenges of using the pain checklist and its clinical relevance to their practice. Participants were also shown the version of the checklist that they did not use, and were asked to make comments on it and to state a preference for which checklist they would use in their clinical practice. For the interviews, the following guide was employed:

(1) Orientation - Review informed consent; explain audio-recording and confidentiality; clarify goals for interview.

(2) Discussion questions:

a. What were your general experiences with using the tool and/or the pain graph?

b. What was difficult or challenging? What was helpful or beneficial?

c. How were your residents' behaviours reflected by the tool?

d. How were the items of the tool?

i. Possible prompts: Were there items that were confusing/vague/inappropriate etc.? Did the items represent pain well or not well for residents with dementia? Did items overlap with other conditions for residents with dementia (e.g., delirium)?

e. How clinically useful was the tool? 
i. Possible prompts: Any comments on length of tool? Time taken to administer? Any comments on organization of the tool (i.e., how items displayed or listed)?

(3) After discussing the tool used, the participant was the other version of the PACSLAC that he or she did not use (i.e., if she used the PACSLAC-II, then she was shown the PACSLAC and vice versa), and was asked about which tool he or she preferred.

(4) Researcher summarized the discussion and asked if there were additional comments.

2.2.4 Expectation. Based on revisions completed on the PACSLAC, it was anticipated that LTC nurses and special care aides would rate the PACSLAC-II as more clinically useful, when compared to the PACSLAC.

2.2.5 Analysis of Ratings and Interview Data. Staff ratings of the clinical utility (e.g., How clinically useful was this tool? How easy was this tool to use? etc.) of the tool they used was analyzed using PASW Statistics 18.0.0 (2009). The tools' average ratings on a scale of 0 to 5 were high overall (e.g., How clinically useful was this tool?, PACSLAC $M=3.50, S D=1.16$; PACSLAC-II $M=4.09, S D=.70$ ). However, results from t-tests revealed that the PACSLAC and PACSLAC-II ratings did not differ significantly (Table 2.10). T-tests comparing nurse versus care aide ratings (Table 2.11) were also performed and found that that ratings differed only on one question, How easy was this tool to use? with nurses rating the tools as easier to use.

Interviews were transcribed and coded using QSR NVivo 8.0 software (QSR International Ltd., 2006) and analyzed using thematic analysis (Braun \& Clarke, 2006; 
Table 2.10 Responses to questions concerning tool clinical utility

\begin{tabular}{|c|c|c|c|c|c|c|}
\hline Question & Tool used & Means & $S D$ & $d f$ & $t$ & $P$ \\
\hline How clinically useful was & PACSLAC & 3.50 & 1.16 & 23 & -1.49 & .15 \\
\hline this tool? & PACSLAC-II & 4.09 & .70 & & & \\
\hline How easy was this tool to & PACSLAC & 4.07 & 1.38 & 24 & -1.17 & .26 \\
\hline use? & PACSLAC-II & 4.58 & .67 & & & \\
\hline How user-friendly was & PACSLAC & 4.00 & 1.36 & 24 & -1.22 & .24 \\
\hline this tool? & PACSLAC-II & 4.54 & .78 & & & \\
\hline How well did the signs in & PACSLAC & 3.64 & 1.01 & 24 & -1.22 & .23 \\
\hline the tool describe the & PACSLAC-II & 4.08 & .79 & & & \\
\hline \multicolumn{7}{|l|}{ behaviors of your patients } \\
\hline \multicolumn{7}{|l|}{ with dementia when } \\
\hline \multicolumn{7}{|l|}{ they're in pain? } \\
\hline How willing would you be & PACSLAC & 3.86 & 1.23 & 24 & -.74 & .47 \\
\hline to use the tool in practice & PACSLAC-II & 4.17 & .83 & & & \\
\hline
\end{tabular}

Note. PACSLAC users $n=14$; PACSLAC-II users $n=12$. 
Table 2.11 Responses to questions concerning tool clinical utility

\begin{tabular}{|c|c|c|c|c|c|c|}
\hline Question & Staff & Mean & $S D$ & $d f$ & $t$ & $P$ \\
\hline How clinically useful was & Nurses & 3.94 & .77 & 23 & 1.02 & .33 \\
\hline this tool? & Care aides & 3.44 & 1.33 & & & \\
\hline How easy was this tool to & Nurses & 4.69 & .60 & 24 & 2.38 & .03 \\
\hline use? & Care aides & 3.70 & 1.49 & & & \\
\hline How user-friendly was & Nurses & 4.47 & .81 & 24 & 1.25 & .22 \\
\hline this tool? & Care aides & 3.90 & 1.52 & & & \\
\hline How well did the signs in & Nurses & 4.06 & .85 & 24 & 1.55 & .13 \\
\hline the tool describe the & Care aides & 3.50 & .97 & & & \\
\hline \multicolumn{7}{|l|}{ behaviors of your patients } \\
\hline \multicolumn{7}{|l|}{ with dementia when } \\
\hline \multicolumn{7}{|l|}{ they're in pain? } \\
\hline How willing would you be & Nurses & 4.19 & .83 & 24 & 1.15 & .26 \\
\hline to use the tool in practice & Care aides & 3.70 & 1.34 & & & \\
\hline
\end{tabular}

Note. Special care aides $n=10$; Nurses $n=16$. 
Graneheim \& Lundman, 2004). Consistent with Braun and Clarke (2006), steps to the thematic analysis were as follows. In the first phase of analysis, the researcher became familiarized with the data by transcribing interviews and re-reading interview transcripts. In the second phase of the analysis, an initial coding structure was created after reviewing related qualitative research (e.g., Bostrom, Kajermo, Nordstrom \& Wallin, 2008; Resnick, Quinn \& Baxter, 2004 etc.) as well as based on the research question of whether the pain checklists were clinically useful to the participants and relative preference for each checklist. Meaning units (i.e., small units of text relating to the same central meaning, Graneheim \& Lundman, 2004) from the interview data were categorized based on whether they referred to the checklist as being clinically useful (e.g., easy to administer, relevant to practice, useful items), not clinically useful (e.g., difficult to administer, irrelevant to practice, not useful items), or whether meaning units reflected other content (e.g., preference for PACSLAC or PACSLAC-II, ambivalence about the checklist, suggestions for changes). This led to a third, inductive phase of coding, where the initial categories were reconceptualized into new categories and subcategories that better fit the data, while addressing the research question. The resultant categories were preference (i.e., for the PACSLAC or PACSLAC-II), barriers to using the tool and facilitators to using the tool. The barriers category consisted of 4 subcategories: differential diagnosis of pain, no new knowledge/no effect on practice, critiques, and systemic barriers. The facilitators category consisted of 3 subcategories: ease of administration, enhanced/informed practice, and potential uses. Descriptions of categories, as well as inclusion and exclusion criteria are detailed in the code book (Appendix F). 
In order to ensure trustworthiness (i.e., that the findings are credible, dependable and transferable; Krefting, 1991), a second coder was provided with the code book and coded a randomly selected $20 \%$ of the meaning units into the established categories and subcategories. The percent agreement between the first and second coders was $83.6 \%$. In addition, the first and second coders then discussed the coding decisions and any disagreements in coding were resolved by consensus. Following this, meaning units in each category were condensed for each interview thus allowing for the final step of comparison across two groups, i.e., participants who used the PACSLAC versus those who used the PACSLAC-II (Boeije, 2002). Upon completion of analysis, member checking was conducted for a randomly selected number of participants $(n=6$, i.e., $20 \%$ of sample) in order to ensure accurate interpretation of the interviews (Gagnon, Hadjistavropoulos \& Williams, in press; Granheim \& Lundman, 2004). Members were asked to review a summary of key findings and provide a response regarding the researcher's interpretation of the interview data. Members responded that the summaries were ${ }^{1}$ largely consistent with their experiences. Any issues that the nurses indicated were missing from the summary were considered and it was determined that none of the new information provided added material that was not already captured by the results and discussion included herein.

\subsubsection{Results of the comparison based on tool used}

2.2.6.1 Preference. A major aim of the staff interviews was to determine the relative clinical usefulness of the PACSLAC and the PACSLAC-II. Comparison of staff

\footnotetext{
${ }^{1}$ Members rated on a scale of 1 to 7 the how well the summaries of key findings captured their own experience of using the pain checklist. Average rating for the preference category was 5.7/7, for facilitators 6.8/7, and for barriers 4.2/7. Most enthusiastic agreement was on the facilitators category, while members responded that they did not experience all of the barriers summarized and emphasized that pain assessment is important despite the barriers.
} 
responses in the Preference category showed that a majority (i.e., 11 out of 14 participants) of those who used the original PACSLAC stated that they preferred the PACSLAC-II when they were shown the revised tool. In contrast, when participants who used the PACSLAC-II were shown the original PACSLAC and asked for their preference, about half the participants stated that they preferred the original PACSLAC and the other half the PACSLAC-II. Interestingly, nurses tended to prefer the PACSLAC-II while care aides tended to favour the original PACSLAC.

Regardless of whether participants used the PACSLAC or PACSLAC-II, when participants reported that they prefer the PACSLAC-II, they cited its length as an important practical advantage. Participants described the shorter tool as being a "quicker glance” which led or would lead to two main benefits, of having fewer behaviours to look for in the resident, and being more likely to be implemented into daily charting.

“Well, for time, I would take the shorter one. For one, it’s smaller, it’s a quick glance. And here [PACSLAC] it's like two sides of the page. And I know from watching other people chart, lots of people don't do a lot of charting, so the shorter the version the better, at least you have something to go by for pain management.” (Care aide, used the PACSLAC)

Also regardless of whether the PACSLAC or PACSLAC-II was used, participants expressed that the PACSLAC-II was shorter and more user-friendly in the LTC setting, and that PACSLAC-II items covered the "main idea” of the original version.

In contrast, those participants who did prefer the original PACSLAC stated that it provided "a bigger picture” of the resident and was a good "reminder" to observe a greater range of behaviours that could potentially indicate pain. Some participants also favoured 
the original PACSLAC because of the inclusion of specific items that were deleted in the revision due to overlap with other conditions such as delirium. Specifically, participants referred to items related to aggression, agitation, and items in the Social Personality and Mood section, and explained that the PACSLAC was preferred because these behaviours were potentially related to pain and important signs for eliminating pain as a possible cause of the behaviour. Further, they reported that the original PACSLAC gave the caregiver "more options” in explaining and describing resident behaviours.

"Whereas this smaller one [PACSLAC-II], yes it was easy to use, but I had to sometimes really think. Especially because I had picked someone that called out but didn't cry. And that was like, okay is that crying or is that... and in the end I did use that, just because it was like crying but using words, not tears. So, that's the thing where I see the older one [PACSLAC] would have been something that would be easier.” (Care aide, used PACSLAC-II)

2.2.6.2 Facilitators and barriers. With respect to the categories of facilitators and barriers to using the tools, major similarities as well as subtle differences were noted when comparing comments referring to the PACSLAC versus the PACSLAC-II (Figure 2.1). In particular, the comparison highlighted each tool's potentially unique contribution to pain assessment for the LTC caregiver. It seemed, for the participants, the original PACSLAC was a more comprehensive tool that called the caregiver's attention to a greater range of possible pain behaviours and may fit within a more general observational assessment of the resident. On the other hand, the PACSLAC-II was described as a tool that due to its length facilitated documentation which could lead to greater focus and 
Figure 2.1 Similarities and differences on the facilitators category

\section{Ease of administration}

- Quick, simple, easy and self-explanatory

- Wording was easy to understand; anybody can use it

\begin{tabular}{|l|l|}
\multicolumn{1}{|c|}{ PACSLAC } & \multicolumn{1}{c|}{ PACSLAC-II } \\
\hline $\begin{array}{l}\text { - Comprehensive, "short but } \\
\text { detailed," "lists all the signs" }\end{array}$ & $\begin{array}{l}\text { Promotes documentation } \\
\text { Concise, "almost went by } \\
\text { memory" }\end{array}$ \\
\hline
\end{tabular}

\section{Enhances/Informs practice}

- Increased awareness of pain behaviours and pain pattern

- Changed communication between staff and pain management for residents

\section{PACSLAC}

- Reminder to not take familiar behaviours as "normal"

- Helped connect mood and personality to pain

\section{PACSLAC-II}

- Made pain measurable

- Promotes consistency across staff, and quickens response to resident pain

\section{Potential uses}

- To use tools as a team

- To use regularly and also under special circumstances e.g., medication changes

\section{PACSLAC}

- Teaching tool

- Add comments section, patient history, and medication

- "Pocket” version

\section{PACSLAC-II}

- With pain graph, as justification for medication decisions

- Add other items, e.g. verbal outbursts, undressing, changes in appetite and sleep etc. 
efficiency in pain control. While these differences in the facilitators for using the PACSLAC and PACSLAC-II emerged, major similarities in this category were also found.

For facilitators for using the PACSLAC and PACSLAC-II, it was apparent that both tools were perceived as quick, easy to understand, and could be used by anyone. Both tools also enhanced or informed the participants' practice by increasing awareness of pain behaviours, promoting more careful observations of residents, increasing awareness of resident pain patterns, as well as changing pain management practices and communication amongst nurses, aides and doctors. As mentioned, in terms of differences, the PACSLAC and PACSLAC-II may each offer unique benefits to pain assessment. The PACSLAC was described as being "short but detailed," which also served as a reminder to not take resident behaviours for granted and to reconsider a greater range of behaviours as potentially due to pain.

"I paid more attention to them and to why they're acting that way. And then I noticed that with the one guy, when he started getting headaches is when he started getting agitated and upset and wanting to go home and more confused, so it kinda all came together.” (Nurse, used PACSLAC)

Participants also commented on the amount of detail in the PACSLAC.

"It covered a wide range of things to look at, which is good because not everybody has the same problem, so there was lots of different areas, or pain expressions, different things that were in the checklist." (Care aide, used PACSLAC) 
On the other hand, the PACSLAC-II was described as a tool which was more condensed and quicker to use, which promoted documentation, and memorization of the pain behaviours with greater use. The organization of the items was also consistent with how observations were done.

“...It seems to me like easier this way...We always do it in section, we always do from head to toe, so this kind of goes down in that same direction and that works well.” (Care aide, used PACSLAC-II)

Those who used the PACSLAC-II also expressed the benefit of being able to make pain concrete and measureable, and along with its short length, promoted documentation and consistency in assessment across staff and across shifts.

"Because before having a checklist like this, there was no way to document how much or if someone's having pain, it was a guess. And this just made it clear to everybody, so everybody's on the same page, everybody's using the same tool. And we all have the same graph. So from shift to shift from day to day, we can track that pain, and we can say definitely there's been a change and we need to change medications maybe or you know lower medications...” (Nurse, used PACSLAC-II)

Participants were also asked to elaborate on how the tools could potentially fit into their clinical practice. These responses were categorized as potential uses and differences in this area between the PACSLAC and PACSLAC-II also emerged. The PACSLAC again was viewed as a comprehensive tool which may fit within a comprehensive approach to assessing a resident, involving the noticing of changes and then ruling out pain and other problems. 
“I'd like to see, okay there's been a change, so what's the first thing we're going to do? And do it systematically. So you don’t have one nurse going, 'Well I think it’s pain, I think it’s this I think it’s that,' you know what I mean? Well if we had like a hierarchy of, okay there's been a change in the patient, okay so now we've checked these two things, we're down to pain, let's all focus this on pain and see if it's that...” (Nurse, used PACSLAC) Specifically, participants suggested that the PACSLAC be used in teaching pain assessment, be carried in a portable pocket version as a reminder of pain behaviours, as well that it include space to include more detailed patient information e.g., medication, patient history, pain patterns etc. On the other hand, it appeared that participants saw the PACSLAC-II as fitting within a more pragmatic and focused approach to pain assessment, where participants commented on the possibility of utilizing the checklist and graph as justification for treatment decisions and requests, with the hope of speeding up the process.

"So this would be a good tool to work for med control for pain control, because it would get us to learn about the residents faster, and their aches and pains faster, and their pain control faster." (Care aide, used PACSLACII)

Participants also hoped that using the PACSLAC-II would lead to other practice changes that could speed up the pain management process.

“... if the staff would know this is going to go farther than just the nurse, and then the doctor. Maybe there's something they're missing. Maybe there's something that they can give for the pain besides what they've 
already prescribed them. That you know, that pain, well pain is pain, you know? Pain control is pretty much...because to see my residents in pain, it hurts. Because you don't know how to touch them, you don't know how to approach them.” (Care aide, used PACSLAC-II)

Suggestions for adding items to the PACSLAC-II were also made, but compared to the suggestions for original version, tended to include specific items that participants felt were relevant but omitted, e.g. changes in appetite or sleep, verbal outbursts etc. Aside from these differences, similarities in how participants thought the PACSLAC or PACLSAC-II could potentially be used were found. For both the PACSLAC and PACLSAC-II, nurses and care aides spoke about the idea of using the tool as a team, e.g. at morning reports where resident pain behaviours can be discussed collaboratively. As well, participants reported various situations where they would like to implement the tools, on a regular basis e.g., weekly, but especially at discrete events, e.g., new admission, injury, medication changes etc.

In terms of barriers to using the tool, major similarities were found for the PACSLAC and PACSLAC-II (Figure 2.2). Regardless of whether the PACSLAC or PACSLAC-II was used, participants stated that diagnosis of pain in residents with dementia and difficulty with communication was extremely challenging. Pain-related items overlapping with other conditions were barriers that arose for both tools.

"So say with pain, shaking and trembling, would I really associate that with pain? Not necessarily. Depends on the situation. Is this person just hypoglycemic? There's a lot more to it, then just saying it's pain. So sure I 
Figure 2.2 Similarities on the barriers category

\section{Differential diagnosis of pain}

- Items overlap with conditions other than pain

\section{No new knowledge/No effect on practice}

- Documentation is not needed

- Staff already understands the resident's pain

- Brief moments of pain does not need treatment

\section{Critiques}

- Variance of scores across staff members

- Certain items did not apply to residents or certain important indicators of pain were missing:

\section{Systemic barriers}

- Not enough time

- Burden of documentation

- Inconsistent shift schedules

- Facility level resistance to new tools

- Nurses do not do personal care and care aides are not responsible for medications 
can check it off, it's present, but is it the pain?. So you're going to have to be smart that way and evaluate.” (Nurse, used PACSLAC) Participants using the PACSLAC-II also voiced similar concerns.

"The facial expressions that could be other things. Crying could be something else too. Yeah some of the body movements, refusing to move, could be just not cooperating because of the dementia. Clenching fist could be anger.” (Nurse, used PACSLAC-II)

As well, not gaining new knowledge nor having an effect on their practice were other common barriers, such as when participants had already conducted informal observational assessments mentally and felt that there was little value in documenting pain scores. Other common critiques for both tools were also identified: the concern of variance of scores across different staff, items that did not apply to their residents, and missing items.

“...the other concern I had was that it didn’t really work with the ones I had simply because their dementia was quite advanced. So they were quite contractured, their fists were clenched, they were in the fetal position, there was more toward the disease process than pain I thought.” (Nurse, used PACSLAC)

Subtle differences were identified in what participants perceived to be missing from each tool. Participants felt that the PACSLAC ought to include more information about pain such as pain site, other physical signs like "swelling of joints," whereas participants felt that the PACSLAC-II ought to include items that were related to but not exclusive to pain, e.g., agitation, as a cue that "makes you think and it makes you look at the person and try and figure it out" (Care aide, used PACSLAC-II). Finally, across both groups systemic 
barriers were reported as being challenges to implementing the tool. These factors related to lack of time, burden of paperwork, inconsistent schedules, facility resistance to new tools, and responsibility of pain assessment (e.g., nurses perceive care aides to be responsible for observation during hands-on care, and care aides perceive nurses to be responsible for pain medication).

\section{Discussion}

Research focusing on refinement and improvement of methods designed to better assess pain in LTC is critical in helping address the problem of pain underassessment and undermanagement that is well documented in such facilities (e.g., Herr, Bjoro, et al., 2006; Herr et al., 2010; Zwakhalen et al., 2006b). Previous research, including both empirical assessment instrument comparisons and reviews of the literature, has shown that the PACSLAC is one of the leading methods for assessing pain in seniors with dementia (e.g., Aubin et al., 2008; Lints-Martindale et al., 2012; Zwakhalen et al., 2006a; 2006b). Moreover, the PACSLAC is the only assessment tool of its kind that comprehensively surveys all of the six pain assessment domains deemed to be important by the AGS (AGS Panel on Persistent Pain in Older Persons, 2002; Hadjistavropoulos, Hunter \& DeverFitzgerald, 2009). Recognizing also that most pain assessment tools developed for seniors with dementia assess behaviours that overlap with delirium (which could be due to causes other than pain), the goal was to develop a revised PACSLAC that minimizes the number of items that overlap with delirium while retaining coverage of all AGS pain assessment domains. Past research (Lints-Martindale et al., 2012) has shown that elimination of items overlapping with delirium did not affect the ability of the PACSLAC to discriminate between pain-related and non-pain-related states. 
The work described herein was also responsive to the need for a shorter, yet comprehensive, pain assessment tool for seniors with dementia. As such, this study led to the development and validation of a shorter and modified version of the PACSLAC, with better ability to discriminate between pain and non-pain states than pre-existing tools including the PACSLAC. The resulting PACSLAC-II, with the advantages of being 31 items in length (as compared to 60 items in the PACSLAC), and including a larger proportion of pain-specific items (as opposed to items that overlap with delirium), was examined using videos of patients with dementia experiencing pain. Use of the tool in a clinical setting was also attempted with frontline nurses and care aides who worked directly with older persons with moderate to severe dementia. The purpose of the clinical implementation was to solicit front line staff opinions about the PACSLAC-II as compared to the PACSLAC. Findings and implications from the three phases of this study (i.e., empirical and theoretically driven revisions, initial validation, and LTC pilot and feedback from front line staff) are discussed in greater detail.

\subsection{Revisions, Validation and Implications}

As indicated earlier, revisions to the PACSLAC were initially motivated by three specific issues related to the tool. First, although the tool takes approximately 5 minutes to administer and was preferred by nursing stuff than shorter tools (Zwakhalen et al., 2006a) front line clinicians often expressed concern about its length of 60 items (Herr et al., 2010). As such, it was decided that a shorter tool, which would still retain or improve on the original PACSLAC's validity would be preferable. A second issue related to the observation that some of the PACSLAC items (as is the case with most other screening assessment tools for seniors with dementia) overlapped with symptoms of delirium which 
could be caused by factors unrelated to pain (e.g., Hadjistavropoulos et al., 2008). A recent study conducted by Lints-Martindale and colleagues (2012) demonstrated that removal of items overlapping in pain and delirium constructs (e.g., agitation, confusion) did not decrease the ability of the tool to discriminate pain from non-pain states. This finding suggested the possibility of removing these items while retaining the tool's validity. Finally, recent research on facial actions while pain is experienced (e.g., lowered brow, raised cheeks) showed that well-defined facial action descriptions tend not to be included in existing observational pain assessment tools (instead non-specific descriptors such as “dirty look” were included). As such, a goal of the revision was to include specific facial actions demonstrated to be useful in previous research on pain states (Craig et al., 2011). Finally, the revision aimed to maintain one of the PACSLAC's strengths which was to continue to cover all of the six pain assessment domains deemed as important by the AGS (2002). In fact, PASCLAC-II items were reorganized to fall under the 6 AGS domains.

Following the theoretically-based development of the PACSLAC-II, pre-existing pain-related video footage was recoded using the new tool. PACSLAC-II scores were then compared to scores from other pain assessment tools for seniors with dementia. Internal consistency and inter-rater reliability of the new tool were found to be satisfactory. Moreover, as hypothesized, not only did the PACSLAC-II discriminate between pain and non-pain-related states bur also accounted for unique variance over and above the contribution of all other tools combined, including the PACSLAC. This suggests that eliminating items overlapping with delirium, and adding more pain-specific items (e.g., specific facial actions) led to greater ability to discriminate between pain and 
non-pain states. It is also possible that reduction of length and re-organization of items into the six AGS-recommended domains (e.g., facial expressions, verbalizations and vocalizations, body movements, etc.) facilitated the process of identifying pain behaviours.

In order to determine whether the extent to which deletion of items most related to delirium accounted for the improved validity, results of this investigation could be compared to results obtained by Lints-Martindale and colleagues (2012), who examined the original PACSLAC excluding delirium-related items but without the addition of any new items. Analyses testing the differentiation of pain versus baseline while taking into account the contribution of other tools (Table 2.5) show that the effect sizes of the PACSLAC-II are greater than those of the PACSLAC (with delirium-items removed; i.e., PACSLAC-II partial $\eta^{2}=.04$ vaccination condition, partial $\eta^{2}=.14$ movementexacerbated pain condition, compared to PACSLAC partial $\eta^{2}=.02$ vaccination condition, partial $\left.\eta^{2}=.01\right)$. Arguably, the PACSLAC-II was able to account for a greater proportion of the variance compared to the original PACSLAC without delirium-related items. This indicates that removing delirium-related items alone from the original PACSLAC did not lead to all of the changes in psychometric properties observed in this study. That is, there were more factors (presumably the clearer pain-specific items) that accounted for the superior ability of the PACSLAC-II to discriminate between pain and non-pain-related states.

Exploratory analyses of each subscales' ability to differentiate across pain and non pain-related states were also conducted to further investigate the contribution of new items (Table 2.8 \& 2.9). As with the overall PACSLAC-II score, facial expressions and body 
movement subscales were able to differentiate across certain conditions, and accounted for similar amounts of variance than the overall score. Nonetheless, this analysis was exploratory and for this reason, it would be premature to advocate for the use of subscale scores other than for descriptive purposes. Moreover, low internal consistencies of subscales also suggest that clinical decisions should be made based on total scale scores. Regardless, the finding concerning the potency of the facial items is consistent with arguments made in the literature about the major contribution of facial behaviour to the communication of pain (Sheu et al., 2008).

The finding that the revision of items led to improved ability to discriminate pain has significant implications. As mentioned in previous studies (e.g., Lints-Martindale et al., 2012), many observational pain assessment tools consist of items that overlap with other conditions such as delirium, mood, dementia etc (e.g., Hadjistavropoulos et al., 2008.). While these items (e.g., agitation) may on the surface appear to facilitate pain detection since the behaviours in question occur during pain states (although they are not unique to pain), the findings of this study suggest that elimination of such items does not decrease the tools' ability to discriminate between pain and non-pain states. Further, the potential appropriateness of shortening the pain checklist by removing delirium-related items is also significant with respect to clinical utility, in that this may reduce the burden of paperwork and time needed for assessment, thus making it more likely that pain management for residents with dementia would be successful.

\subsection{Front Line Use}

In order to examine front line staff impressions about the tool, this investigation involved feedback from LTC staff who used the PACSLAC or the PACSLAC-II with 
their residents with dementia. It was anticipated that, based on the reduction and modification of items, LTC staff would prefer the PACSLAC-II over the original version. Indeed, many LTC nurses and care aides preferred the PACSLAC-II due to its length and condensed nature, which in their view may promote documentation and greater efficiency in pain assessment and management. The PACSLAC-II also appeared to be consistent with a more focused approach to pain assessment where staff used the tool to identify increases and decrease in pain after injury or medication changes. Other benefits for using the PACSLAC-II, as well as the PACSLAC, were also reported. However, a smaller number of nurses and care aides reported preference for the original PACSLAC. Specifically, they expressed that they favoured the PACSLAC because it provided a greater range of options for describing possible pain, which helped them rule out pain as an underlying cause of observed resident behaviours. This advantage, in their view, also outweighed its relatively longer format. Thus, it appeared that the PACSLAC may represent for nurses and care aides a more comprehensive and open approach to pain assessment which may possibly be most appropriate for training of new staff. Interestingly, participants indicated the desire to keep the shortened version but at the same time add items such as aggression or anxiety, which were eliminated due to its nonpain specific nature. Despite this staff impression, the psychometric examination of the tools indicated that the shorter version had better discriminative properties. In future, it may be possible for to address this suggestion by rewording the last item of the PACSLAC-II, without adding items that are not specific to pain. Specifically, a participant indicated that item 31, i.e., Are there mental status changes that are due to pain and are not explained by another condition (e.g., delirium due to medication, etc.), was confusing 
and that the definition for mental status needed to be clarified. This may indicate that the term mental status may not be user-friendly to LTC nurses and care aides, and that some nurses and care aides may not be aware that item 31 can be used to describe agitation, aggression, or change in mood that is suspected to be due to pain and not explained by any other condition, such as delirium.

\subsection{Clinical Directions}

It has been suggested that pain assessment in LTC should be regular and individualized (Hadjstavropoulos, Dever-Fitzgerald \& Marchildon, 2010) and that this can be accommodated using minimal extra resources (Hadjistavropoulos, Janzen-Claude, Hadjistavropoulos, March ildon, Kaasalainen, Gallagher et al., 2011). It is hoped that the refinement of the PACSLAC accomplished in this investigation will make the PACSLACII easier to implement due to its reduced length and improved validity. As indicated by the front line staff feedback, an advantage of a briefer tool is that it may lead to greater efficiency in pain assessment and management, which would ultimately improve the quality of life of LTC residents with dementia. Nonetheless, it would be important for research on the PACSLAC-II to continue with a focus on validity in different settings (e.g., acute care) as well as clinical utility. Moreover, it would be important to encourage further validation work by other research teams. It would also be necessary to collect PACSLAC-II data from LTC staff in order to determine its reliability and validity when used outside the scientific laboratory. In previous implementation studies of the PACSLAC, it was shown that where the PACSLAC was systemically used by LTC staff, pain management practices improved (Fuchs-Lacelle et al., 2006; Hadjistavropoulos, Kaasalainen, Williams \& Zacharias, submitted). It would be important to replicate these 
findings using the PACSLAC-II. Studies have also tested other observational pain assessment tools' ability to discriminate between pre- and post-medication administration behaviours (e.g., Lane et al., 2003; Warden et al., 2003). This examination would also be required to provide potential support for using the PACSLAC-II as a tool to facilitate the complex decisions involved in the management of medications. Another issue that is often related to assessment scales is the use of cut-off scores (e.g., Zwakhalen, van der Steen \& Najim, 2012). Cut-off scores can be an excellent tool that aides the clinician in making decisions about treatment. Though in the case of PACSLAC-II pain assessment in older persons with dementia, cut-off scores are not recommended due to lack of knowledge about pain in dementia, as well as the serious implications for medical decision-making. That is, dementia represents very diverse types of damage to the brain with some types of dementia leading to increased responsiveness to pain (e.g., dementias primarily affecting the frontal lobe) versus less pronounced responsiveness in other dementias (e.g., Snowden, Bathgate, Varma, Blackshaw, Gibbons \& Neary, 2001). Therefore, to assume that a single cut-off score would be suitable for all people regardless of dementia type and severity would be problematic. Moreover, scores on these types of scales are affected by type of pain (e.g., acute injury or exacerbation of chronic pain) and duration of observation (e.g., moment of care or number of hours) that would also make the use of such scores problematic. As well, an individual who normally exhibits many behaviours would likely have a higher baseline score compared to an individual who is more contracted and has limited movement. This range of scores in baseline states makes it difficult to establish that a certain score, or cut-off score, would mean a certain level of pain for most, if not all individuals with dementia. At the present time, the approach that is recommended for pain 
assessment in older persons is an individualized one that involves regular pain assessments. In other words, pain assessment should be ongoing (and conducted under similar conditions over time) in LTC facilities (Hadjistavropoulos et al., 2007). Using regular assessment, pain would be indicated based on deviations from the individual's normal pattern of behaviours, or baseline score, rather than comparing the individual's score to others' in the population (Hadjistavropoulos et al., 2007).

Another important line of future inquiry would involve LTC implementation studies using the PACSLAC-II. Specifically, this issue relates to feedback from front line staff regarding the use of pain checklists within a team. LTC nurses and care aides described the possibility of using the checklist for one patient collaboratively (e.g., nurse asking care aides if behaviors were seen), as well as having multiple assessors use the checklist consecutively (e.g., whoever is on shift would complete an assessment). These were ways that staff considered utilizing the PACSLAC or PACSLAC-II in order to overcome barriers of lack of time during their shift, or the barrier of not working with one patient consistently. Aside from collecting inter-rater statistics, previous studies of the PACSLAC in LTC settings have involved the same nurse assessor for each resident with dementia, which may be problematic as this does not reflect the reality of shift rotations and staff assignment to different residents at different times. Thus, in order to further examine the clinical utility of the PACSLAC-II, it would be worthwhile to provide support for collaborative use of the PACSLAC-II, with a focus on reliability and validity in clinical settings. Careful training of staff to ensure cross-individual consistency in item endorsement rules would be required. 
Finally, it is notable that, when looking at preference for the PACSLAC and PACSLAC-II, more care aides preferred the PACSLAC, while more nurses preferred the revised version. This may be due to a number of reasons. First, it may be that because care aides interact with residents most and thus are responsible for the largest part of observation of any sign of discomfort, they would prefer a larger tool that signals a need to further investigation. They would then report this sign to the nurse who would be responsible for making decisions regarding medication or other interventions. Consistent with the nurses' preference for the PACSLAC-II, is its quicker and more focused approach. Another reason for the care aids’ preference for the PACSLAC may be that they receive far less education on pain and dementia than nurses, and that having a more comprehensive list of possible pain behaviours facilitates their observation of patients and alerts them to consider behaviours that they take for granted as due to dementia. This is also consistent with the feedback that the PACSLAC would make a good teaching tool for training in pain assessment. These findings indicate that participants' feedback regarding a tool's clinical utility is potentially related to but not entirely dependent on factors external to the tool's psychometric properties, such as staff education and perceived responsibility for pain management. Whether a pain tool is considered clinically useful to a LTC nurse or care aide is also related to these external factors. It is crucial that researchers not only attend to the psychometric properties but also the factors influencing the clinical utility of these observational pain assessment tools, because improving pain management for residents dementia require that the tools fit with and enhance the work of LTC staff. 


\section{Conclusion}

Underassessment and undermanagement of pain in older persons with dementia is a serious concern in LTC facilities. While the problem is multifaceted, a large part may be due to the barrier in pain communication due to the residents' decline in verbal ability and lack of recognition of pain in this population. Observational assessment tools have become one way of overcoming this barrier. This study was aimed at revising a well established, psychometrically valid tool, the PACSLAC. Revisions were conducted in order to create a shorter version with more items that were specific to pain. The resultant PACSLAC-II has demonstrated positive psychometric properties and, in particular, the ability to differentiate between pain and non-pain states over and above other tools of its kind, including the original PACSLAC. LTC staff who used the PACSLAC-II and PACSLAC also report benefits of its shortened format. These findings have significant implications for the study of observational pain assessment tools for older persons with dementia, as they suggest that removal of non-pain specific items and addition of more pain-specific items may increase the tools’ ability to identify pain. Moreover, it is hoped that the shorter tool will increase the probability of successful implementation of permanent evidencebased pain assessment protocols in LTC, and ultimately improvement of pain management for residents with dementia. 


\section{References}

Abbey, J., Piller, N., De Bellis, A., Esterman, A., Parker, D., Giles, L., \& Lowcay, B. (2004). The Abbey pain scale: a 1-minute numerical indicator for people with end-stage dementia. International Journal of Palliative Nursing, 10(1), 6-13. Retrieved from http://www.internurse.com

Afzal, N., Buhagiar, K., Flood, J., \& Cosgrave, M. (2010). Quality of end-of-life care for dementia patients during acute hospital admission: A retrospective study in Ireland. General Hospital Psychiatry, 32(2), 141-146. doi:10.1016/j.genhosppsych.2009.10.003

Alexander, B. J., Plank, P., Carlson, M. B., Hanson, P., Picken, K., \& Schwebke, K. (2005). Methods of pain assessment in residents of long-term care facilities: A pilot study. Journal of the American Medical Directors Association, 6(2), 137143. doi:10.1016/j.jamda.2004.12.024

Alexopoulos, G. S., Abrams, R. C., Young, R. C., \& Shamoian, C. A. (1988). Cornell Scale for Depression in Dementia. Biological Psychiatry, 23(3), 271-284. doi:10.1016/0006-3223(88)90038-8

Allcock, N., McGarry, J., \& Elkan, R. (2002). Management of pain in older people within the nursing home: A preliminary study. Health \& Social Care in the Community, 10(6), 464-471. doi:10.1046/j.1365-2524.2002.00392.x

Altman, D. G. (1991). Practical Statistics for Medical Research. London: Chapman and Hall.

Alzheimer Society of Canada. (2010). Rising tide: The impact of dementia on Canadian Society. 
American Geriatric Society Panel on Persistent Pain in Older Persons. (2002). The Management of persistent pain in older persons. Journal of the American Geriatrics Society, 50(S6), 205-224. doi:10.1046/j.1532-5415.50.6s.1.x

American Psychiatric Association (2000). Diagnostic and Statistical Manual of Mental Disorders (4th ed., text rev.). Washington D. C.: American Psychiatric Pub, Inc. Asmundson, G. J. G., \& Wright, K. D. (2004). Biopsychosocial approaches to pain. In T. Hadjistavropoulos \& K. D. Craig (Eds.) Pain: Psychological Perspectives (pp. 35-58). Mahwah, NJ: Lawrence Erlbaum.

Aubin, M., Adolfsson, R., Savoie, M., Lemay, S., Hadjistavropoulos, T., Fillion, L., Beaulieu, M., et al. (2008). Validité et utilité clinique d’une grille d’observation (PACSLAC-F) pour évaluer la douleur chez des aînés atteints de démence vivant en milieu de soins de longue durée. Canadian Journal on Aging, 27(1), 45-55. doi:10.3138/cja.27.1.045

Benedetti, F., Arduino, C., Vighetti, S., Asteggiano, G., Tarenzi, L., \& Rainero, I. (2004). Pain reactivity in Alzheimer patients with different degrees of cognitive impairment and brain electrical activity deterioration. Pain, 111(1-2), 22-29. doi:10.1016/j.pain.2004.05.015

Benedetti, F., Vighetti, S., Ricco, C., Lagna, E., Bergamasco, B., Pinessi, L., \& Rainero, I. (1999). Pain threshold and tolerance in Alzheimer’s disease. Pain, 80(1-2), 377382. doi:10.1016/0006-3223(88)90038-8

Bergman, S. (2007). Management of musculoskeletal pain. Best Practice \& Research Clinical Rheumatology, 21(1), 153-166. doi:10.1016/j.berh.2006.10.001 
Berthier, M., Starkstein, S., \& Leiguarda, R. (1988). Asymbolia for pain: A sensorylimbic disconnection syndrome. Annals of Neurology, 24(1), 41-49. doi:10.1002/ana.410240109

Biersdorff, K. K. (1994). Incidence of significantly altered pain experience among individuals with developmental disabilities. American Journal of Mental Retardation, 98(5), 619-631.

Bland, J. M., \& Altman, D. G. (1997). Statistics notes: Cronbach’s alpha. BMJ, 314-572. doi:http://dx.doi.org/10.1136/bmj.314.7080.572

Blennow, K., de Leon, M. J., \& Zetterberg, H. (2006). Alzheimer’s disease. The Lancet, 368(9533), 387-403. doi:10.1016/S0140-6736(06)69113-7

Blomqvist, K., \& Hallberg, I. R. (2001). Recognising pain in older adults living in sheltered accommodation: The views of nurses and older adults. International Journal of Nursing Studies, 38(3), 305-318. doi:10.1016/S0020-7489(00)00078-X

Boeije, H. (2002). A purposeful approach to the constant comparative method in analyses of qualitative interviews. Quality \& Quantity, 36, 391-409.

Boyatzis, R. E. (1998). Transforming qualitative information: thematic analysis and code development. Thousand Oaks, CA: Sage.

Braun, V., \& Clarke, V. (2006). Using thematic analysis in psychology. Qualitative Research in Psychology, 3(2), 77-101. doi:10.1191/1478088706qp063oa

Brodaty, H., Draper, B., \& Low, L. (2003). Nursing home staff attitudes towards residents with dementia: Strain and satisfaction with work. Journal of Advanced Nursing, 44(6), 583-590. doi:10.1046/j.0309-2402.2003.02848.x 
Buffum, M. D., Miaskowski, C., Sands, L., \& Brod, M. (2001). A pilot study of the relationship between discomfort and agitation in patients with dementia. Geriatric Nursing, 22(2), 80-85. doi:10.1067/mgn.2001.115196

Burgener, S., \& Twigg, P. (2002). Relationships among caregiver factors and quality of life in care recipients with irreversible dementia. Alzheimer Disease and Associated Disorders, 16(2), 88-102. doi:10.1097/00002093-200204000-00006

Canadian Psychological Association (2000). Canadian Code of Ethics for Psychologists: ( $3^{\text {rd }}$ ed.). Ottawa, ON: Canadian Psychological Association.

Cariaga, J., Burgio, L., Flynn, W., \& Martin, D. (1991). A controlled study of disruptive vocalizations among geriatric residents in nursing homes. Journal of the American Geriatrics Society, 39(5), 501-507.

Cheung, G., \& Choi, P. (2008). The use of the Pain Assessment Checklist for Seniors with Limited Ability to Communicate (PACSLAC) by caregivers in dementia care. The New Zealand Medical Journal, 121(1286), 21-29. Retrieved from http://journal.nzma.org.nz

Cipher, D. J., \& Clifford, P. A. (2004). Dementia, pain, depression, behavioral disturbances, and ADLs: toward a comprehensive conceptualization of quality of life in long-term care. International Journal of Geriatric Psychiatry, 19(8), 741748. doi:10.1002/gps.1155

Cipher, D. J., Clifford, P. A., \& Roper, K. D. (2006). Behavioral manifestations of pain in the demented elderly. Journal of the American Medical Directors Association, 7(6), 355-365. doi:10.1016/j.jamda.2005.11.012 
Closs, S. J., Cash, K., Barr, B., \& Briggs, M. (2005). Cues for the identification of pain in nursing home residents. International Journal of Nursing Studies, 42(1), 3-12. doi:10.1016/j.ijnurstu.2004.05.012

Cole, L. J., Farrell, M. J., Duff, E. P., Barber, J. B., Egan, G. F., \& Gibson, S. J. (2006). Pain sensitivity and fMRI pain-related brain activity in Alzheimer's disease. Brain, 129(11), 2957-2965. doi:10.1093/brain/awl228

Collett, B., O’Mahoney, S., Schofield, P., Closs, S. J., \& Potter, J. (2007). The assessment of pain in older people. Clinical Medicine, 7(5), 496-500. doi: 10.7861/clinmedicine.7-5-496

Conn, D., \& Thorpe, L. (2007). Assessment of behavioural and psychological symptoms associated with dementia. The Canadian Journal of Neurological Sciences, 34 Suppl 1, S67-71. Retrieved from http://cjns.metapress.com

Cope, D. K. (2009). Intellectual milestones in our understanding and treatment of pain. In S. M. Fishman, J. C. Ballantyne, \& J. P. Rathmell (Eds). Bonica's Management of Pain (pp. 1-13). New York: Lippincott Williams and Wilkins.

Costardi, D., Rozzini, L., Costanzi, C., Ghianda, D., Franzoni, S., Padovani, A., \& Trabucchi, M. (2007). The Italian version of the pain assessment in advanced dementia (PAINAD) scale. Archives of Gerontology and Geriatrics, 44(2), 175180. doi:10.1016/j.archger.2006.04.008

Craig, K. D. (2009). The Social Communication Model of Pain. Canadian Psychology, 50(1), 22-32. doi:10.1037/a0014772 
Craig, K. D., \& Patrick, C. J. (1985). Facial expression during induced pain. Journal of Personality and Social Psychology, 48(4), 1080-1091. doi:10.1037/00223514.48.4.1080

Craig, K. D., Hyde, S. A., \& Patrick, C. J. (1991). Genuine, suppressed and faked facial behavior during exacerbation of chronic low back pain. Pain, 46(2), 161-171. doi:10.1016/0304-3959(91)90071-5

Craig, K. D., Prkachin, K. M., \& Grunau, R. E. (2011). The facial expression of pain. In D. C. Turk \& R. Melzack (Eds.) Handbook of Pain Assessment (3rd ed., pp. 117133). New York: Guilford Press.

de Rond, M. E., de Wit, R., van Dam, F. S., van Campen, B. T., den Hartog, Y. M., \& Klievink, R. M. (2000). A pain monitoring program for nurses: effects on nurses’ pain knowledge and attitude. Journal of Pain and Symptom Management, 19(6), 457-467. doi:10.1016/S0885-3924(00)00128-7

Defrin, R., Lotan, M., \& Pick, C. G. (2006). The evaluation of acute pain in individuals with cognitive impairment: A differential effect of the level of impairment. Pain, 124(3), 312-320. doi:10.1016/j.pain.2006.04.031

DeWaters, T., Faut-Callahan, M., McCann, J. J., Paice, J. A., Fogg, L., Hollinger-Smith, L., Sikorski, K., et al. (2008). Comparison of self-reported pain and the PAINAD scale in hospitalized cognitively impaired and intact older adults after hip fracture surgery. $\quad$ Orthopaedic Nursing, 27(1), 21-28. doi: 10.1097/01.NOR.0000310607.62624.74 
Douzjian, M., Wilson, C., Shultz, M., Berger, J., Tapnio, J., \& Blanton, V. (1998). A program to use pain control medication to reduce psychotropic drug use in residents with difficult behavior. Annals of Long-Term Care, 6(5), 174-179.

Duffy, B., Oyebode, J. R., \& Allen, J. (2009). Burnout among care staff for older adults with dementia. Dementia, 8(4), 515 -541. doi:10.1177/1471301209350285

Edberg, A., \& Hallberg, I. R. (2001). Actions seen as demanding in patients with severe dementia during one year of intervention. Comparison with controls. International Journal of Nursing Studies, 38(3), 271-285. doi:10.1016/S00207489(00)00076-6

Ekman, P. (1993). Facial expression and emotion. American Psychologist, 48(4), 384392. doi:10.1037//0003-066X.48.4.384

Ekman, P., \& Friesen, W. (1978). Facial Action Coding System: A Technique for the Measurement of Facial Movement. Palo Alto, Ca.: Consulting Psychologists Press.

Eritz, H., \& Hadjistavropoulos, T. (2010). Do informal caregivers consider nonverbal behavior when they assess pain in people with severe dementia? The Journal of Pain. doi:10.1016/j.jpain.2010.07.012

Feldt, K. S. (2000). The Checklist of Nonverbal Pain Indicators (CNPI). Pain Management Nursing, 1(1), 13-21. doi:10.1053/jpmn.2000.5831

Feldt, K. S., Ryden, M. B., \& Miles, S. (1998). Treatment of pain in cognitively impaired compared with cognitively intact older patients with hip-fracture. Journal of the American Geriatrics Society, 46(9), 1079-1085. 
Ferrell, B. A., Ferrell, B. R., \& Rivera, L. (1995). Pain in cognitively impaired nursing home patients. Journal of Pain and Symptom Management, 10(8), 591-598. doi:10.1016/0885-3924(95)00121-2

Fick, D. M., Agostini, J. V., \& Inouye, S. K. (2002). Delirium superimposed on dementia: a systematic review. Journal of the American Geriatrics Society, 50(10), 1723-1732. doi:10.1046/j.1532-5415.2002.50468.x

Foley, D. C., \& McCutcheon, H. (2004). Detecting pain in people with an intellectual disability. Accident and Emergency Nursing, 12(4), 196-200. doi:16/j.aaen.2004.06.002

Follick, M. J., Ahern, D. K., \& Aberger, E. W. (1985). Development of an audiovisual taxonomy of pain behavior: Reliability and discriminant validity. Health Psychology, 4(6), 555-568. doi:10.1037/0278-6133.4.6.555

Folstein, M. F., Folstein, S. E., \& McHugh, P. R. (1975). “Mini-mental state”. A practical method for grading the cognitive state of patients for the clinician. Journal of Psychiatric Research, 12(3), 189-198. Retrieved from http://www.sciencedirect.com

Fordyce, W. E. (1976). Behavioral methods for chronic pain and illness. Saint Louis: Mosby.

Frank, L., Kleinman, L., Ciesla, G., Rupnow, M. F. T., \& Brodaty, H. (2004). The effect of risperidone on nursing burden associated with caring for patients with dementia. Journal of the American Geriatrics Society, 52(9), 1449-1455. doi:10.1111/j.1532-5415.2004.52406.x 
Fuchs-Lacelle, S., \& Hadjistavropoulos, T. (2004). Development and preliminary validation of the pain assessment checklist for seniors with limited ability to communicate (PACSLAC). Pain Management Nursing, 5(1), 37-49. doi:10.1016/j.pmn.2003.10.001

Fuchs-Lacelle, S., \& Hadjistavropoulos, T. (2005). A checklist for pain assessment in LTC. Canadian Nursing Home, 16, 4-17.

Fuchs-Lacelle, S., Hadjistavropoulos, T., \& Lix, L. (2008). Pain assessment as intervention: a study of older adults with severe dementia. The Clinical Journal of Pain, 24(8), 697-707. doi:10.1097/AJP.0b013e318172625a

Fuchs-Lacelle, S., Hadjistavropoulos, T., Sharpe, D., Williams, J., Martin, R., \& LaChapelle, D. (2003). Comparing two observational systems in the assessment of knee pain. Pain Research \& Management, 8(4), 205-211. Retrieved from http://www.pulsus.com

Gagliese, L., \& Katz, J. (2000). Medically unexplained pain is not caused by psychopathology. Pain Research and Management. Retrieved from http://www.pulsus.com

Gagnon, M. M., Hadjistavropoulos, T., \& Williams, J. (in press). Development and mixed methods evaluation of a pain assessment video training program for longterm care staff. Pain Research \& Management.

Gibson, S. J., Voukelatos, X., Ames, D., Flicker, L., \& Helme, R. D. (2001). An examination of pain perception and cerebral event-related potentials following carbon dioxide laser stimulation in patients with Alzheimer's disease and age- 
matched control volunteers. Pain Research and Management, 6(3), 126-132. Retrieved from http://www.pulsus.com

Graneheim, U. H., \& Lundman, B. (2004). Qualitative content analysis in nursing research: concepts, procedures and measures to achieve trustworthiness. Nurse Education Today, 24, 105-112. doi: 10.1016/j.nedt.2003.10.001

Hadjistavropoulos, T., \& Craig, K. D. (2002). A theoretical framework for understanding self-report and observational measures of pain: A communications model. Behaviour Research and Therapy, 40(5), 551-570. doi:10.1016/S00057967(01)00072-9

Hadjistavropoulos, T., \& Craig, K. D. (Eds.) (2004). Pain: Psychological Perspectives. Mahwah, NJ: Lawrence Erlbaum.

Hadjistavropoulos, T., Breau, L. M., \& Craig, K. D. (2011). Assessment of pain in adults and children with limited ability to communicate. In D. C. Turk \& R. Melzack (Eds.) Handbook of Pain Assessment (3rd ed., pp. 260-280). New York: Guilford Press.

Hadjistavropoulos, T., Craig, K. D., \& Fuchs-Lacelle, S. (2004). Social influences and the communication of pain. In T. Hadjistavropoulos \& K. D. Craig (Eds.) Pain: Psychological Perspectives (pp. 87-112). Mahwah, NJ: Lawrence Erlbaum.

Hadjistavropoulos, T., Craig, K. D., Duck, S., Cano, A., Goubert, L., Jackson, P. L., Mogil, J. S., et al. (2011). A biopsychosocial formulation of pain communication. Psychological Bulletin. doi:10.1037/a0023876 
Hadjistavropoulos, T., Fitzgerald, T. D., \& Marchildon, G. P. (2010). Practice guidelines for assessing pain in older persons with dementia residing in long-term care facilities. Physiotherapy Canada, 62(2), 104-113. doi:10.3138/physio.62.2.104

Hadjistavropoulos, T., Gibson, S., \& Fine, P. G. (2011). Pain in older persons: A brief clinical guide. In M. E. Lynch, K. D. Craig \& P. W. H. Peng (Eds.) Clinical Pain Management: A Practical Guide (pp. 311-318). Hoboken, NJ: Wiley-Blackwell.

Hadjistavropoulos, T., Herr, K., Turk, D. C., Fine, P. G., Dworkin, R. H., Helme, R., Jackson, K., et al. (2007). An interdisciplinary expert consensus statement on assessment of pain in older persons. The Clinical Journal of Pain, 23(Supplement 1), S1-S43. doi:10.1097/AJP.0b013e31802be869

Hadjistavropoulos, T., Hunter, P. \& Dever-Fitzgerald, T. (2009). Pain assessment and management in older adults: Conceptual issues and clinical challenges. Canadian Psychology, 50, 241-254. doi: 10.1037/a0015341

Hadjistavropoulos, T., Janzen Claude, J. A., Hadjistavropoulos, H. D., Marchildon, G. P., Kaasalainen, S., Gallagher, R., \& Beattie, B. L. (2011). Stakeholder opinions on a transformational model of pain management in long-term care. Journal of Gerontological Nursing, 37, 40-51. doi: 10.3928/00989134-20100503-03

Hadjistavropoulos, T., Kaasalainen, S., Williams, J., \& Zacharias, R. (submitted). Improving pain assessment practices and outcomes in long-term care facilities: A mixed methods investigation. Manuscript submitted for publication.

Hadjistavropoulos, T., LaChapelle, D. L., Hadjistavropoulos, H. D., Green, S., \& Asmundson, G. J. G. (2002). Using facial expressions to assess musculoskeletal 
pain in older persons. European Journal of Pain, 6(3), 179-187. doi:10.1053/eujp.2001.0327

Hadjistavropoulos, T., LaChapelle, D. L., MacLeod, F. K., Snider, B., \& Craig, K. D. (2000). Measuring movement-exacerbated pain in cognitively impaired frail elders. The Clinical Journal of Pain, 16(1), 54-63. doi:10.1097/00002508200003000-00009

Hadjistavropoulos, T., Voyer, P., Sharpe, D., Verreault, R., \& Aubin, M. (2008). Assessing pain in dementia patients with comorbid delirium and/or depression. Pain Management Nursing, 9(2), 48-54. doi:10.1016/j.pmn.2007.12.004

Hale, C. J., \& Hadjistavropoulos, T. (1997). Emotional components of pain. Pain Research and Management, 2, 217-225. Retrieved from http://www.pulsus.com

Hedeker, D., \& Gibbons, R. D. (2006). Longitudinal Data Analysis. Hoboken, N. J.: John Wiley and Sons.

Herr, K., Bjoro, K., \& Decker, S. (2006). Tools for assessment of pain in nonverbal older adults with dementia: A state-of-the-science review. Journal of Pain and Symptom Management, 31(2), 170-192. doi:10.1016/j.jpainsymman.2005.07.001

Herr, K., Bursch, H., Ersek, M., Miller, L. L., \& Swafford, K. (2010). Use of painbehavioral assessment tools in the nursing home. Journal of Gerontological Nursing, 36(3), 18-29. doi:10.3928/00989134-20100108-04

Herr, K., Coyne, P. J., Key, T., Manworren, R., McCaffery, M., Merkel, S., Pelosi-Kelly, J., et al. (2006). Pain assessment in the nonverbal patient: Position statement with clinical practice recommendations. Pain Management Nursing, 7(2), 44-52. doi:10.1016/j.pmn.2006.02.003 
Herr, K., Titler, M. G., Schilling, M. L., Marsh, J. L., Xie, X., Ardery, G., Clarke, W. R., et al. (2004). Evidence-based assessment of acute pain in older adults: current nursing practices and perceived barriers. The Clinical Journal of Pain, 20(5), 331. Retrieved from http://ovidsp.tx.ovid.com.libproxy.uregina.ca

Hill, A. (1999). Phantom limb pain: A review of the literature on attributes and potential mechanisms. Journal of Pain and Symptom Management, 17(2), 125-142. doi:10.1016/S0885-3924(98)00136-5

Hobara, M. (2005). Beliefs about appropriate pain behavior: cross-cultural and sex differences between Japanese and Euro-Americans. European Journal of Pain, 9(4), 389-393. doi:10.1016/j.ejpain.2004.09.006

Horgas, A. L., Nichols, A. L., Schapson, C. A., \& Vietes, K. (2007). Assessing pain in persons with dementia: Relationships among the non-communicative patient's pain assessment instrument, self-report, and behavioral observations. Pain Management Nursing, 8(2), 77-85. doi:10.1016/j.pmn.2007.03.003

Husebo, B. S., Strand, L. I., Moe-Nilssen, R., Husebo, S. B., \& Ljunggren, A. E. (2009). Pain behaviour and pain intensity in older persons with severe dementia: reliability of the MOBID Pain Scale by video uptake. Scandinavian Journal of Caring Sciences, 23(1), 180-189. doi:10.1111/j.1471-6712.2008.00606.x

Jensen, I. B., Bradley, L. A., \& Linton, S. J. (1989). Validation of an observation method of pain assessment in non-chronic back pain. Pain, 39(3), 267-274. doi:10.1016/0304-3959(89)90039-0 
Julien, N., \& Marchand, S. (2006). Endogenous pain inhibitory systems activated by spatial summation are opioid-mediated. Neuroscience Letters, 401(3), 256-260. doi:16/j.neulet.2006.03.032

Julien, N., Goffaux, P., Arsenault, P., \& Marchand, S. (2005). Widespread pain in fibromyalgia is related to a deficit of endogenous pain inhibition. Pain, 114(1-2), 295-302. doi:10.1016/j.pain.2004.12.032

Kaasalainen, S., Coker, E., Dolovich, L., Papaioannou, A., Hadjistavropoulos, T., Emili, A., \& Ploeg, J. (2007). Pain management decision making among long-term care physicians and nurses. Western Journal of Nursing Research, 29(5), 561-580. doi:10.1177/0193945906295522

Kaasalainen, S., Middleton, J., Knezacek, S., Hartley, T., Stewart, N., Ife, C., \& Robinson, L. (1998). Pain and cognitive status in the institutionalized elderly: perceptions \& interventions. Journal of Gerontological Nursing, 24(8), 24-31; quiz 50-51.

Kajermo, K. N., Bostrom, A., Thompson, D. S., Hutchinson, A. M., Estabrooks, C. A., \& Wallin, L. (2010). The BARRIERS scale- the barriers to research utilization scale: A systematic review. Implementation Science, 5, 32. doi:10.1186/1748-5908-5-32

Kappesser, J., \& Williams, A. C. (2002). Pain and negative emotions in the face: judgements by health care professionals. Pain, 99(1-2), 197-206. doi:10.1016/S0304-3959(02)00101-X

Kappesser, J., Williams, A. C., \& Prkachin, K. M. (2006). Testing two accounts of pain underestimation. Pain, 124(1-2), 109-116. doi:10.1016/j.pain.2006.04.003 
Katsma, D. L., \& Souza, C. H. (2000). Elderly pain assessment and pain management knowledge of long-term care nurses. Pain Management Nursing, 1(3), 88-95. doi:10.1053/jpmn.2000.9294

Keefe, F. J., \& Block, A. R. (1982). Development of an observation method for assessing pain behavior in chronic low back pain patients. Behavior Therapy, 13(4), 363375. doi:10.1016/S0005-7894(82)80001-4

Keefe, F. J., \& Smith, S. (2002). The assessment of pain behavior: Implications for applied psychophysiology and future research directions. Applied Psychophysiology and Biofeedback, 27(2), 117-127. doi: 10.1023/A:1016240126437

Keefe, F. J., Bradley, L. A., \& Crisson, J. E. (1990). Behavioral assessment of low back pain: Identification of pain behavior subgroups. Pain, 40(2), 153-160. doi:10.1016/0304-3959(90)90066-M

Keefe, F. J., Caldwell, D. S., Queen, K., Gil, K. M., Martinez, S., Crisson, J. E., Ogden, W., et al. (1987). Osteoarthritic knee pain: A behavioral analysis. Pain, 28(3), 309-321.

Keefe, F. J., Somers, T. J., Williams, D. A., \& Smith, S. J. (2011). Assessment of pain behaviors. In D. C. Turk \& R. Melzack (Eds.) Handbook of Pain Assessment (3rd ed., pp. 134-150). New York: Guilford Press.

Kelley, A. S., Siegler, E. L., \& Reid, M. C. (2008). Pitfalls and recommendations regarding the management of acute pain among hospitalized patients with dementia. Pain Medicine, 9(5), 581-586. doi:10.1111/j.1526-4637.2008.00472.x 
Kellow, J. T., \& Wilson, V. L. (2008). Setting standards and establishing cut scores on criterion-referenced assessments: Some technical and practical considerations. In J. W. Osborne (Ed.). Best practices in quantitative methods (pp. 15-28). Thousand Oaks: Sage.

Kovach, C. R., Noonan, P. E., Griffie, J., Muchka, S., \& Weissman, D. E. (2001). Use of the assessment of discomfort in dementia protocol. Applied Nursing Research, 14(4), 193-200. doi:10.1053/apnr.2001.26784

Kovach, C. R., Noonan, P. E., Griffie, J., Muchka, S., \& Weissman, D. E. (2002). The assessment of discomfort in dementia protocol. Pain Management Nursing, 3(1), 16-27. doi:10.1053/jpmn.2002.30389

Kovach, C. R., Noonan, P. E., Schlidt, A. M., \& Wells, T. (2005). A model of consequences of need-driven, dementia-compromised behavior. Journal of Nursing Scholarship, 37(2), 134-140. doi:10.1111/j.1547-5069.2005.00025_1.x

Kovach, C. R., Weissman, D. E., Griffie, J., Matson, S., \& Muchka, S. (1999). Assessment and treatment of discomfort for people with late-stage dementia. Journal of Pain and Symptom Management, 18(6), 412-419. doi:10.1016/S08853924(99)00094-9

Kovach, C., Griffie, J., Muchka, S., Noonan, P., \& Weissman, D. (2000). Nurses’ perceptions of pain assessment and treatment in the cognitively impaired elderly: It's not a guessing game. Clinical Nurse Specialist, 14(5), 215-220. Retrieved from http://ovidsp.tx.ovid.com.libproxy.uregina.ca

Krefting, L. (1991). Rigor in qualitative research: the assessment of trustworthiness. American Journal of Occupational Therapy, 45, 214-222. 
Kunz, M., Gruber, A., \& Lautenbacher, S. (2006). Sex differences in facial encoding of pain. The Journal of Pain, 7(12), 915-928. doi:10.1016/j.jpain.2006.04.012

Kunz, M., Mylius, V., Scharmann, S., Schepelman, K., \& Lautenbacher, S. (2009). Influence of dementia on multiple components of pain. European Journal of Pain, 13(3), 317-325. doi:10.1016/j.ejpain.2008.05.001

Kunz, M., Mylius, V., Schepelmann, K., \& Lautenbacher, S. (2008). Impact of age on the facial expression of pain. Journal of Psychosomatic Research, 64(3), 311-318. doi:10.1016/j.jpsychores.2007.09.010

Kunz, M., Mylius, V., Schepelmann, K., \& Lautenbacher, S. (2009). Effects of age and mild cognitive impairment on the pain response system. Gerontology, 55(6), 674682. doi:10.1159/000235719

Kunz, M., Scharmann, S., Hemmeter, U., Schepelmann, K., \& Lautenbacher, S. (2007). The facial expression of pain in patients with dementia. Pain, 133(1-3), 221-228. doi:10.1016/j.pain.2007.09.007

Lane, P., Kuntupis, M., MacDonald, S., McCarthy, P., Panke, J., Warden, V., \& Volicer, L. (2003). A pain assessment tool for people with advanced Alzheimer's and other progressive dementias. Home Healthcare Nurse, 21(1), 32-37. doi:10.1097/00004045-200301000-00007

Laurila, J. V., Pitkala, K. H., Strandberg, T. E., \& Tilvis, R. S. (2004). Detection and documentation of dementia and delirium in acute geriatric wards. General Hospital Psychiatry, 26(1), 31-35. doi:10.1016/j.genhosppsych.2003.08.003 
Lemay, M., \& Landreville, P. (2010). Verbal agitation in dementia: The role of discomfort. American Journal of Alzheimer's Disease and Other Dementias, 25(3), 193-201. doi: 10.1177/1533317509356687

Leong, I. Y., Chong, M. S., \& Gibson, S. J. (2006). The use of a self-reported pain measure, a nurse-reported pain measure and the PAINAD in nursing home residents with moderate and severe dementia: a validation study. Age and Ageing, 35(3), 252-256. doi:10.1093/ageing/afj058

Lerner, A. J., Hedera, P., Koss, E., Stucky, J., \& Friedland, R. P. (1997). Delirium in Alzheimer Disease. Alzheimer Disease and Associated Disorders, 11(1), 16-20. doi:10.1097/00002093-199703000-00004

Lints-Martindale, A. C. (2010). An examination of observational pain assessment tools for dementia patients and the role of delirium. University of Regina, Regina.

Lints-Martindale, A. C., Hadjistavropoulos, T., Barber, B., \& Gibson, S. J. (2007). A psychophysical investigation of the Facial Action Coding System as an index of pain variability among older adults with and without Alzheimer’s Disease. Pain Medicine, 8(8), 678-689. doi:10.1111/j.1526-4637.2007.00358.x

Lints-Martindale, A. C., Hadjistavropoulos, T., Lix, L. M., \& Thorpe, L. (2012). A comparative investigation of observational pain assessment tools for older adults with dementia. Clinical Journal of Pain, 28, 226-237. doi: 10.1097/AJP.0b013e3182290d90

Liu, J. Y. W., Briggs, M., \& Closs, S. J. (2010). The Psychometric Qualities of Four Observational Pain Tools (OPTs) for the assessment of pain in elderly people with 
osteoarthritic pain. Journal of Pain and Symptom Management, 40(4), 582-598. doi:10.1016/j.jpainsymman.2010.02.022

Lix, L. M., \& Fouladi, R. T. (2007). Robust step-down tests for multivariate independent group designs. British Journal of Mathematical and Statistical Psychology, 60, 245-265. doi: 10.1348/000711006X117853

Manfredi, P. L., Breuer, B., Meier, D. E., \& Libow, L. (2003). Pain assessment in elderly patients with severe dementia. Journal of Pain and Symptom Management, 25(1), 48-52. doi:10.1016/S0885-3924(02)00530-4

Marzinski, L. R. (1991). The tragedy of dementia: Clinically assessing pain in the confused nonverbal elderly. Journal of Gerontological Nursing, 17(6), 25-28.

Masters Steedman, S., Middaugh, S. J., Kee, W. G., Carson, D. S., Harden, R. N., \& Miller, M. C. (1992). Chronic-pain medications: equivalence levels and method of quantifying usage. The Clinical Journal of Pain, 8(3), 204-214.

McCaffery, M., Ferrell, B. R., \& Pasero, C. (2000). Nurses' personal opinions about patients' pain and their effect on recorded assessments and titration of opioid doses. Pain Management Nursing, 1(3), 79-87. doi:10.1053/jpmn.2000.9295

McDonald, M. (1999). Assessment and management of cancer pain in the cognitively impaired elderly. Geriatric Nursing, 20(5), 249-253. doi:10.1016/S01974572(99)70023-6

McGrath, P. A., Seifert, C. E., Speechley, K. N., Booth, J. C., Stitt, L., \& Gibson, M. C. (1996). A new analogue scale for assessing children's pain: An initial validation study. Pain, 64(3), 435-443. doi:10.1016/0304-3959(95)00171-9 
Melzack, R. W. P., \& Wall, P. (1965). Pain mechanisms: A new theory. Science, 78, 101-107.

Melzack, R., \& Katz, J. (2004). The gate control theory: Reaching for the brain. In T. Hadjstavropoulos \& K. D. Craig (Eds.) Pain: Psychological Perspectives (pp. 1334). Mahwah, NJ: Lawrence Erlbaum.

Mentes, J. C., Teer, J., \& Cadogan, M. P. (2004). The pain experience of cognitively impaired nursing home residents: Perceptions of family members and certified nursing assistants. Pain Management Nursing, 5(3), 118-125. doi:10.1016/j.pmn.2004.01.001

Merskey, H., \& Bogduk, N. (1994). Classification of Chronic Pain: Descriptions of Chronic Pain Syndromes and Definitions of Pain Terms (2nd ed.). Seattle: IASP Press.

Mezinskis, P. M., Keller, A. W., \& Luggen, A. S. (2004). Assessment of pain in the cognitively impaired older adult in long-term care. Geriatric Nursing, 25(2), 107112. doi:10.1016/j.gerinurse.2003.09.005

Middleton, J., Knezacek, S., Robinson, L., Hartley, T., \& Kaasalainen, S. (1997). An exploratory study of pain in the institutionalized elderly. American Journal of Alzheimer's Disease and Other Dementias, 12(4), 159-166. doi:http://dx.doi.org/10.1177/153331759701200403

Miller, L. L., Nelson, L. L., \& Mezey, M. (2000). Comfort and pain relief in dementia: Awakening a new beneficence. Journal of Gerontological Nursing, 26(9), 32-40.

Morello, R., Jean, A., Alix, M., Sellin-Peres, D., \& Fermanian, J. (2007). A scale to measure pain in non-verbally communicating older patients: the EPCA-2 Study of 
its psychometric properties. Pain, 133(1-3), 87-98. doi:10.1016/j.pain.2007.03.007

Morgan, D. G., Stewart, N. J., D’Arcy, C., Forbes, D., \& Lawson, J. (2005). Work stress and physical assault of nursing aides in rural nursing homes with and without dementia special care units. Journal of Psychiatric and Mental Health Nursing, 12(3), 347-358. doi:10.1111/j.1365-2850.2005.00846.x

Morley, S., Williams, A., \& Hussain, S. (2008). Estimating the clinical effectiveness of cognitive behavioural therapy in the clinic: Evaluation of a CBT informed pain management programme. Pain, 137(3), 670-680. doi:16/j.pain.2008.02.025

Morrison, R. S., \& Siu, A. L. (2000). A comparison of pain and its treatment in advanced dementia and cognitively intact patients with hip fracture. Journal of Pain and Symptom Management, 19(4), 240-248. doi:10.1016/S0885-3924(00)00113-5

Mylius, V., Kunz, M., Hennighausen, E., Lautenbacher, S., \& Schepelmann, K. (2008). Effects of ageing on spinal motor and autonomic pain responses. Neuroscience Letters, 446(2-3), 129-132. doi:10.1016/j.neulet.2008.09.048

Nygaard, H. A., \& Jarland, M. (2006). The Checklist of Nonverbal Pain Indicators (CNPI): testing of reliability and validity in Norwegian nursing homes. Age and Ageing, 35(1), 79-81. doi:10.1093/ageing/afj008

Oosterman, J. M., de Vries, K., Dijkerman, H. C., de Haan, E. H. F., \& Scherder, E. J. A. (2008). Exploring the relationship between cognition and self-reported pain in residents of homes for the elderly. International Psychogeriatrics, 21(01), 157163. doi:10.1017/S1041610208007941 
Park, J., Castellanos-Brown, K., \& Belcher, J. (2009). A review of observational pain scales in nonverbal elderly with cognitive impairements. Research on Social Work Practice, 20(6), 651-664. doi:10.1177/1049731508329394

Parmalee, P. A. (1996). Pain in cognitively impaired older persons. Clinics in Geriatric Medicine, 12(3), 473-487.

Patrick, C. J., Craig, K. D., \& Prkachin, K. M. (1986). Observer judgments of acute pain: Facial action determinants. Journal of Personality and Social Psychology, 50(6), 1291-1298. doi:10.1037/0022-3514.50.6.1291

Pautex, S., Herrmann, F., Le Lous, P., Fabjan, M., Michel, J., \& Gold, G. (2005). Feasibility and reliability of four pain self-assessment scales and correlation with an observational rating scale in hospitalized elderly demented patients. The Journals of Gerontology Series A: Biological Sciences and Medical Sciences, 60(4), 524 -529. doi:10.1093/gerona/60.4.524

Pillai Riddell, R. R., \& Craig, K. D. (2007). Judgments of infant pain: The impact of caregiver identity and infant age. Journal of Pediatric Psychology, 32(5), 501511. doi:10.1093/jpepsy/jsl049

Portenoy, R. K., Ugarte, C., Fuller, I., \& Haas, G. (2004). Population-based survey of pain in the United States: Differences among white, African American, and Hispanic subjects. The Journal of Pain, 5(6), 317-328. doi:10.1016/j.jpain.2004.05.005

Porter, F. L., Malhotra, K. M., Wolf, C. M., Morris, J. C., Miller, J. P., \& Smith, M. C. (1996). Dementia and response to pain in the elderly. Pain, 68(2-3), 413-421. doi:10.1016/S0304-3959(96)03210-1 
Prkachin, K. M. (1992). The consistency of facial expressions of pain: a comparison across modalities. Pain, 51(3), 297-306. doi:10.1016/0304-3959(92)90213-U

Prkachin, K. M. (2005). The consistency of facial expressions of pain: A comparison across modalities. In P. Ekman \& E. L. Rosenberg (Eds.) What the Face Reveals: Basic and Applied Studies of Spontaneous Expression using the Facial Action Coding System (FACS) (pp. 181-200). Oxford: Oxford University Press.

Prkachin, K. M., \& Craig, K. D. (1995). Expressing pain: The communication and interpretation of facial pain signals. Journal of Nonverbal Behavior, 19(4), 191205. doi:10.1007/BF02173080

Prkachin, K. M., Berzins, S., \& Mercer, S. R. (1994). Encoding and decoding of pain expressions: A judgement study. Pain, 58(2), 235-259. doi:10.1016/03043959(94)90206-2

Prkachin, K. M., Hughes, E., Schultz, I., Joy, P., \& Hunt, D. (2002). Real-time assessment of pain behavior during clinical assessment of low back pain patients. Pain, 95(1-2), 23-30. doi:10.1016/S0304-3959(01)00369-4

Prkachin, K. M., Solomon, P. E., \& Ross, J. (2007). Underestimation of pain by healthcare providers: Towards a model of the process of inferring pain in others. The Canadian Journal of Nursing Research, 39(2), 88-106. Retrieved from http://digital.library.mcgill.ca/cjnr

Proctor, W. R., \& Hirdes, J. P. (2001). Pain and cognitive status among nursing home residents in Canada. Pain Research and Management, 6(3), 119-125. Retrieved from http://www.pulsus.com

QSR International Ltd. (2008). NVivo (Version 8.0) [Computer software]. Thousand Oaks, CA: Scolari-Sage. 
Rainero, I., Vighetti, S., Bergamasco, B., Pinessi, L., \& Benedetti, F. (2000). Autonomic responses and pain perception in Alzheimer's disease. European Journal of Pain, 4(3), 267-274. doi:10.1053/eujp.2000.0185

Regnard, C., Reynolds, J., Watson, B., Matthews, D., Gibson, L., \& Clarke, C. (2007). Understanding distress in people with severe communication difficulties: developing and assessing the Disability Distress Assessment Tool (DisDAT). Journal of Intellectual Disability Research, 51(4), 277-292. doi:10.1111/j.13652788.2006.00875.x

Resnick, B., Quinn, C., \& Baxter, S. (2004). Testing the feasibility of implementation of clinical practice guidelines in long-term care facilities. Journal of American Medical Directors Association, 5, 1-8. doi: 10.1016/S1525-8610(04)70037-7

Reynolds, K. S., Hanson, L. C., DeVellis, R. F., Henderson, M., \& Steinhauser, K. E. (2008). Disparities in pain management between cognitively intact and cognitively impaired nursing home residents. Journal of Pain and Symptom Management, 35(4), 388-396. doi:10.1016/j.jpainsymman.2008.01.001

Rosenberg, E. L. (2005). The study of spontaneous facial expressions in psychology. In P. Ekman \& E. L. Rosenberg (Eds.) What the Face Reveals: Basic and Applied Studies of Spontaneous Expression using the Facial Action Coding System (FACS) (pp. 3-18). Oxford: Oxford University Press.

Sampson, E. L., Gould, V., Lee, D., \& Blanchard, M. R. (2006). Differences in care received by patients with and without dementia who died during acute hospital admission: a retrospective case note study. Age and Ageing, 35(2), 187-189. doi:10.1093/ageing/afj025 
Scherder, E. J. A., \& Bouma, A. (2000). Visual analogue scales for pain assessment in Alzheimer’s disease. Gerontology, 46(1), 47-53. doi:10.1159/000022133

Scherder, E. J. A., Sergeant, J. A., \& Swaab, D. F. (2003). Pain processing in dementia and its relation to neuropathology. The Lancet Neurology, 2(11), 677-686. doi:10.1016/S1474-4422(03)00556-8

Schuler, M. S., Becker, S., Kaspar, R., Nikolaus, T., Kruse, A., \& Basler, H. D. (2007). Psychometric Properties of the German "Pain Assessment in Advanced Dementia Scale” (PAINAD-G) in Nursing Home Residents. Journal of the American Medical Directors Association, 8(6), 388-395. doi:16/j.jamda.2007.03.002

Schuler, M., Njoo, N., Hestermann, M., Oster, P., \& Hauer, K. (2004). Acute and chronic pain in geriatrics: Clinical characteristics of pain and the influence of cognition. Pain Medicine, 5(3), 253-262. doi:10.1111/j.1526-4637.2004.04040.x

Semla, T. P., Beizer, J. L., \& Higbee, M. D. (2004). Lexi-Comp’s Geriatric Dosage Handbook: Including Clinical Recommendations and Monitoring Guidelines (14th ed.). Hudson, OH: Lexi Comp.

Sengstaken, E., \& King, S. (1993). The problems of pain and its detection among geriatric nursing home residents. Journal of the American Geriatrics Society, 41(5), 541-544. $\quad$ Retrieved from http://onlinelibrary.wiley.com.libproxy.uregina.ca:2048

Shega, J. W., Rudy, T., Keefe, F. J., Perri, L. C., Mengin, O. T., \& Weiner, D. K. (2008). Validity of pain behaviors in persons with mild to moderate cognitive impairment. Journal of the American Geriatrics Society, 56(9), 1631-1637. doi:10.1111/j.1532-5415.2008.01831.x 
Sheu, E., Versloot, J., Nader, R., Kerr, D., \& Craig, K. D. (2011). Pain in the Elderly: Validity of Facial Expression Components of Observational Measures. The Clinical Journal of Pain. doi:10.1097/AJP.0b013e31820f52e1

Silverstein, M. L., \& Nelson, L. D. (2000). Clinical and research implications of revising psychological tests. Psychological Assessment, 12(3), 298-303. doi:10.1037//1040-3590.12.3.298

Simon, D., Craig, K. D., Gosselin, F., Belin, P., \& Rainville, P. (2008). Recognition and discrimination of prototypical dynamic expressions of pain and emotions. Pain, 135(1-2), 55-64. doi:10.1016/j.pain.2007.05.008

Sloane, P. D., Davidson, S., Buckwalter, K., Lindsey, B. A., Ayers, S., Lenker, V., \& Burgio, L. D. (1997). Management of the patient with disruptive vocalization. The $\begin{array}{llll}\text { Gerontologist, } & \text { 37(5), } & \text { 675-682. } & \text { Retrieved }\end{array}$ http://proquest.umi.com.libproxy.uregina.ca:2048

Sloane, P. D., Davidson, S., Knight, N., Tangen, C., \& Mitchell, C. M. (1999). Severe disruptive vocalizers. Journal of the American Geriatrics Society, 47(4), 439-445.

Smith, G. T., \& McCarthy, D. M. (1995). Methodological considerations in the refinement of clinical assessment instruments. Psychological Assessment, 7(3), 300-308. doi:10.1037/1040-3590.7.3.300

Smith, M. (2005). Pain assessment in nonverbal older adults with advanced dementia. Perspectives In Psychiatric Care, 41(3), 99-113. doi:10.1111/j.17446163.2005.00021.x 
Snow, A. L., \& Shuster, J. L. (2006). Assessment and treatment of persistent pain in persons with cognitive and communicative impairment. Journal of Clinical Psychology, 62(11), 1379-1387. doi:10.1002/jclp.20317

Snow, A. L., Weber, J. B., O’Malley, K. J., Cody, M., Beck, C., Bruera, E., Ashton, C., et al. (2004). NOPPAIN: a nursing assistant-administered pain assessment instrument for use in dementia. Dementia and Geriatric Cognitive Disorders, 17(3), 240-246. doi:10.1159/000076446

Snowden, J., Bathgate, D., Varma, A., Blackshaw, A., Gibbons, Z., \& Neary, D. (2001). Distinct behavioural profiles in frontotemporal dementia and semantic dementia. Journal of Neurology, Neurosurgery \& Psychiatry, 70, 323-332. doi:10.1136/jnnp.70.3.323

Solomon, P. E., Prkachin, K. M., \& Farewell, V. (1997). Enhancing sensitivity to facial expression of pain. Pain, 71(3), 279-284. doi:10.1016/S0304-3959(97)03377-0

SPSS etc. (2009). PASW Statistics (Release 18.0.0) [Computer software]. Winwrap Basic, Copyright 1993-2007 Polar Engineering and Consulting.

Stevenson, K. M., Brown, R. L., Dahl, J. L., Ward, S. E., \& Brown, M. S. (2006). The Discomfort Behavior Scale: A measure of discomfort in the cognitively impaired based on the Minimum Data Set 2.0. Research in Nursing and Health, 29(6), 576587. doi:10.1002/nur.20168

Stolee, P., Hillier, L. M., Esbaugh, J., Bol, N., McKellar, L., \& Gauthier, N. (2005). Instruments for the assessment of pain in older Persons with cognitive impairment. Journal of the American Geriatrics Society, 53(2), 319-326. doi:10.1111/j.1532-5415.2005.53121.x 
Sufka, K. J., \& Price, D. D. (2002). Gate control theory reconsidered. Brain and Mind, 3(2), 277-290. Retrieved from http://www.springerlink.com

Turk, D. C. (1996). Psychological aspects of chronic pain and disability. Journal of Musculoskelatal Pain, 4(4), 145-154. doi:10.1300/J094v04n04_14

Turk, D. C. (2001). Physiological and psychological bases of pain. In A. Baum, T. A. Revenson, \& J. E. Singer (Eds.), Handbook of Health Psychology (pp. 117-138).

Turk, D. C., \& Flor, H. (1999). The biobehavioural perspective of pain. In R. J. Gatchel \& D. C. Turk (Eds.), Psychosocial Factors in Pain: Clinical Perspectives (pp. 1834). New York: Guilford Press.

Turk, D. C., Dworkin, R. H., Revicki, D., Harding, G., Burke, L. B., Cella, D., Cleeland, C. S., et al. (2008). Identifying important outcome domains for chronic pain clinical trials: An IMMPACT survey of people with pain. Pain, 137(2), 276-285. doi:16/j.pain.2007.09.002

Turk, D. C., Meichenbaum, D., \& Genest, M. (1983). Pain and behavioural medicine: A cognitive-behavioural perspective. New York: Guilford Press.

van Iersel, T., Timmerman, D., \& Mullie, A. (2006). Introduction of a pain scale for palliative care patients with cognitive impairment. International Journal of Palliative Nursing, 12(2), 54-59. Retrieved from http://www.internurse.com van Nispen tot Pannerden, S. C., Candel, M. J. J. M., Zwakhalen, S. M. G., Hamers, J. P. H., Curfs, L. M. G., \& Berger, M. P. F. (2009). An item response theory-based assessment of the Pain Assessment Checklist for Seniors With Limited Ability to Communicate (PACSLAC). The Journal of Pain, 10(8), 844-853. doi:10.1016/j.jpain.2009.02.007 
Vaughn, S., Schumm, J. S., \& Sinagub, J. (1996). Focus group interivews in education and psychology. Thousand Oaks: Sage.

Villanueva, M. R., Smith, T. L., Erickson, J. S., Lee, A. C., \& Singer, C. M. (2003). Pain Assessment for the Dementing Elderly (PADE): Reliability and validity of a new measure. Journal of the American Medical Directors Association, 4(1), 1-8. doi:10.1097/01.JAM.0000043419.51772.A3

Warden, V., Hurley, A. C., \& Volicer, L. (2003). Development and psychometric evaluation of the Pain Assessment in Advanced Dementia (PAINAD) scale. Journal of the American Medical Directors Association, 4(1), 9-15. doi:10.1097/01.JAM.0000043422.31640.F7

Wary, B., \& Doloplus, C. (1999). Doloplus-2, a scale for pain measurement. Soins. Gérontologie, (19), 25-27. Retrieved from http://www.elsevier.com

Waters, S. J., Riordan, P. A., Keefe, F. J., \& Lefebvre, J. C. (2008). Pain behavior in rheumatoid arthritis patients: Identification of pain behavior subgroups. Journal of Pain and Symptom $\quad$ Management, 36(1), doi:10.1016/j.jpainsymman.2007.08.015

Weiner, D. K., Peterson, B., \& Keefe, F. (1999). Chronic pain-associated behaviors in the nursing home: Resident versus caregiver perceptions. Pain, 80(3), 577-588. doi:10.1016/S0304-3959(98)00249-8

Wynne, C. F., Ling, S. M., \& Remsburg, R. (2000). Comparison of pain assessment instruments in cognitively intact and cognitively impaired nursing home residents. Geriatric Nursing, 21(1), 20-23. doi:10.1067/mgn.2000.105793 
Zillmer, E. A., Spiers, M. V., \& Culbertson, W. C. (2008). Principles of Neuropsychology (2nd ed.). Belmont, CA: Thomson Wadsworth.

Zwakhalen, S. M. G., Hamers, J. P. H., \& Berger, M. P. F. (2006a). The psychometric quality and clinical usefulness of three pain assessment tools for elderly people with dementia. Pain, 126(1-3), 210-220. doi:10.1016/j.pain.2006.06.029

Zwakhalen, S. M. G., Hamers, J. P. H., \& Berger, M. P. F. (2007). Improving the clinical usefulness of a behavioural pain scale for older people with dementia. Journal of Advanced Nursing, 58(5), 493-502. doi:10.1111/j.1365-2648.2007.04255.x

Zwakhalen, S. M. G., Hamers, J. P. H., Abu-Saad, H., \& Berger, M. P. F. (2006b). Pain in elderly people with severe dementia: A systematic review of behavioural pain assessment tools. BMC Geriatrics, 6(1), 3. doi:10.1186/1471-2318-6-3

Zwakhalen, S. M., van der Steen, J. T., \& Najim, M. D. (2012). Which score most likely represents pain on the observational PAINAD pain scale for patients with dementia? Journal of American Medical Directors Association, 13, 384-389. doi: 10.1016/j.jamda.2011.04.002. 


\section{Appendix A}

\begin{tabular}{|c|c|}
\hline \multicolumn{2}{|c|}{ Pain Assessment Checklist for Seniors with } \\
\multicolumn{2}{|c|}{ Limited Ability to Communicate-II (PACSLAC-II) }
\end{tabular}

COPYRIGHT: The PACSLAC-II is copyrighted by Sarah Chan, Thomas Hadjistavropoulos and Shannon Fuchs-Lacelle. For permission to reproduce the PACSLAC contact thomas.hadjistavropoulos@uregina.ca who is authorised to provide permission on behalf of all copyright holders. 


\section{Appendix B}

\section{To Begin the Pain Assessment Study...}

1. Choose at least 3 older patients with whom you regularly work. Please choose patients who are living with moderate to severe dementia.

$>$ Do you have 3 patients with moderate to severe dementia in mind?

2. For these 3 (or more) patients, we are asking you to assess their pain using the pain checklist at least 3 times a week, for 4 weeks. Completion of the checklist typically takes under 5 minutes.

3. You will have a pain assessment package for each patient. The package will contain the pain checklists and a pain graph where you can easily track the patient's pain score over the next 4 weeks.

4. The checklist is a clinical tool that can help inform decisions about patient functioning. Relevant articles about the checklist have been left at the nurses' station at your unit.

5. You may choose to use the pain checklist more than 3 times per week. Extra checklists will be available.

6. At the end of the 4 weeks, you will be contacted about a focus group to give us feedback about assessing pain using the pain checklist.

\section{How to use the Pain Checklist...}

1. The pain checklist requires you to observe your patient before you fill out the checklist. It is recommended that you observe your patient under very similar circumstances and for the same length of time each time you use the pain checklist.

For example, if you decide to observe the patient during a transfer and then fill out the pain checklist, then you should observe a transfer and fill out the checklist for all of the assessments for that patient. If you decide to fill out the checklist based on your observations of the patient over the course of your shift, then you should do the same for all of the checklists.

$>$ Generally, observation during movement is more likely to reveal underlying pain problems.

2. When you fill out the pain checklist, write down the date, the condition of the observation (e.g., shift, transfer, walk, etc.), and add up the number of checkmarks. The total number of checkmarks is the pain score.

3. Put the pain score on the graph in the front of the package, along with the date, so you 
can see how the patient's pain score changes over the next 4 weeks.

4. Write down and date anything that happens to your patient that might be related to the patient's pain score, such as a fall, fever, change of medication etc.

5. There is no cut-off score for this pain checklist because we recognize that people with dementia can show very different behaviours and so have very different scores.

Therefore, it is important to look at each patient and notice whether there are unusual changes in the patient's own pain score. Unusual elevations may be related to increases in pain. Often the administration of clinically appropriate, health-professional approved interventions will result in reductions in pain scores.

6. Consider using the information derived from the checklist to facilitate clinical decisions about the patient. Examples of such decisions are:

Contacting the physician to request that he or she consider a change in the patients' medications, as clinically appropriate.

$>$ Use non-pharmacological pain management strategies (e.g., a revised clinically appropriate repositioning schedule, massage, warm blanket etc).

$>$ Discussion with the personnel clinically responsible for the patient concerning the administration of PRN medications.

\section{When assessing pain, also keep in mind...}

1. Always attempt to ask the patient to self-report on pain, regardless of the level of his or her cognitive functioning.

2. Patient history, lab test results, and environmental, psychological and social factors should also be taken into consideration. For example,

$>$ Does the patient have a condition that could be causing pain?

$>$ Are there test results that can help explain these symptoms?

$>$ How was the patient's mood and interactions with other people?

3. Assessing pain using the pain checklist is a way of screening for pain. The checklist may suggest the presence of pain when there is no pain, or may fail to identify pain when there is pain. Always look at other information that is available to you, such as in the previous point.

4. This pain checklist can be used for evaluating whether pain management interventions (e.g., analgesic, massage, etc.) was effective. For example, the checklist can be used before and after you administer an intervention, and these pain scores can be documented and reviewed later.

5. Be aware that symptoms of delirium overlap with signs of uncontrolled pain (e.g., 
behavioural disturbance). Also be aware that patients can be experiencing delirium and pain at the same time.

\section{Questions?}

Thank you for participating in this study. Your help is greatly appreciated.

Should you have any questions during the course of the study, please feel free to contact

Sarah Chan, at the Health Psychology Laboratory, University of Regina, at (306) 337-2601 or (306) 585-4428 or you can email her at chan204s@uregina.ca. 
Appendix C

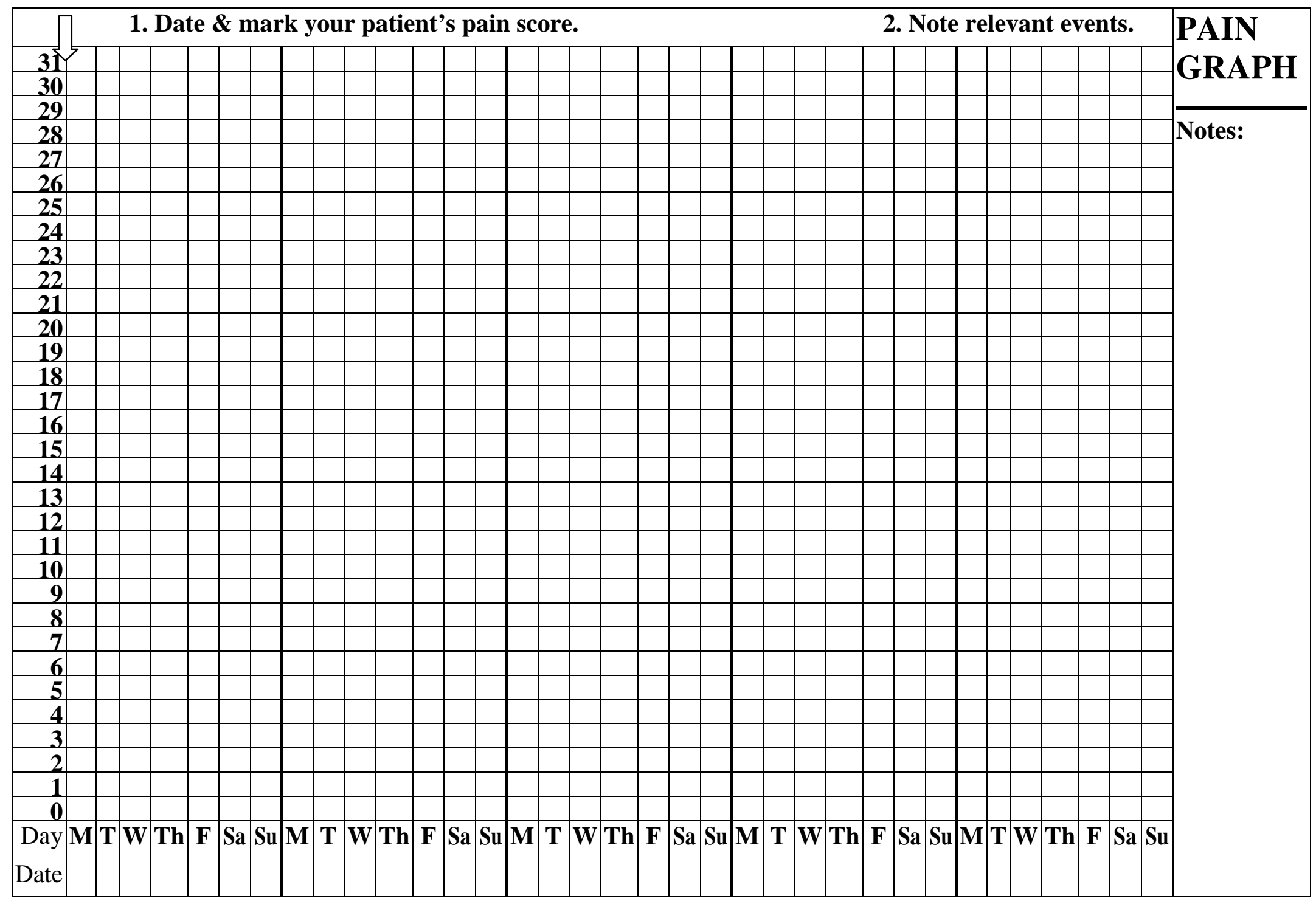




\section{Appendix D}

\section{Staff Participant Information \\ Participant ID:}

1. ___Male _Female

2. Age:

3. Job Title (Position):

4. How many hours do you usually work per week ?

5. Level of Education (Please check):

Less than Grade 12

Grade 12 or equivalent

Post-Secondary education (college, university, technical training) \& Number of

years:

Master's or Doctoral level training \& Number of years:

Other, please describe (include number of years):

6. How many years have you worked at a long-term care facility?

7. How many years have you worked in the health care field?

8. What percentage of the patients you work live with dementia?

9. How many hours of training or education have you received on pain management (e.g., how to identify, document, treat pain) for people with dementia? 


\section{Appendix E}

\section{$\underline{\text { Staff Participant Feedback }}$}

Circle tool used: PACSLAC / PACSLAC-II

On average, how long did it take for you to complete the tool? (minutes)

1. How clinically useful was this tool?

0

Not at all

relevant

2. How easy was this tool to use?
0

1

Not at all easy

3. How user-friendly was this tool?

0

1

Not at all user-

friendly
12

2

3

4

5

Very relevant

4. How well did the signs in the tool describe the behaviours of your patients with dementia when they are in pain?

\section{0}

Not at all well
1
2

2
3

4

5

Very easy

45

Very user-

friendly

5. How willing would you be to use this tool in practice with your patients?
0
1
2
3
4
5
Very willing

Not at all willing

Do you have any additional comments regarding the tool and its usage? 


\section{Appendix F}

\section{Code Book}

\section{BARRIER}

\section{Differential diagnosis of pain}

\begin{tabular}{|l|} 
\\
\hline Systemic factors
\end{tabular}

(dd) These comments refer to how difficult it is for the staff to know with certainty what they are observing in the patient is due to pain or due to some other condition.

\section{Time/Shift/Facility constraints (tsf) - These} comments refer to the difficulty of using the checklist due to the limitations in the system in which they work, for example: lack of time, the nature of shift work, or factors inherent in the facility that is a barrier to pain assessment.

Role confusion (rc) - These comments refer to whether the staff sees the usage of the pain checklist and what might follow from the pain assessment as part of their job or not part of their job. This also includes the staff perceiving the usage of the pain checklist and what might follow from the pain assessment as more appropriately assigned to another staff position.

Note: This category might overlap with Imagined uses, but what distinguishes them is that the comments that fall under Systemic factors/tsf/rc are said in a way that conveys a "barrier", where difficulty and lack of a solution is central. Comments under Imagined uses may mention difficulty but should convey as its central point an idea or potential solution to the problem.

No new knowledge / No effect on practice

(nnk) These comments refer to lack of knowledge gained from using the pain checklist, or lack of change in practice after using the pain checklist; essentially, that using the checklist did not change their perspectives nor the way they worked.

\section{Critiques}

(cr) These comments refer to criticisms related specifically to the checklist, and can include mention of: - items that did not apply to their patients

- how the pain checklist itself is organized, laid out, applied

- whether scores and coding on the pain checklist can be reliable across different users. 


\begin{tabular}{|c|c|}
\hline \multicolumn{2}{|l|}{ FACILITATORS } \\
\hline Ease of administration & $\begin{array}{l}\text { (ea) These comments refer to the ease of using the pain } \\
\text { checklist, and any descriptions of the checklist that } \\
\text { relates to ease of administration. }\end{array}$ \\
\hline $\begin{array}{l}\text { Enhanced / Informed } \\
\text { practice }\end{array}$ & $\begin{array}{l}\text { (en) These comments refer to the relevance of the pain } \\
\text { checklist to their practice. Specifically, these comments } \\
\text { are about: } \\
\text { - how the checklist was accurate/consistent in } \\
\text { identifying pain in patients } \\
\text { - how using the pain checklist led to actual change in } \\
\text { what they saw in the patient, such as enhancing } \\
\text { recognition of pain } \\
\text { - how using the pain checklist led to actual change in } \\
\text { what they did with the patient or with other people } \\
\text { involved at the facility, such as improving pain } \\
\text { management, communication with other staff etc. }\end{array}$ \\
\hline Imagined uses & $\begin{array}{l}\text { (im) These comments refer to how the staff member } \\
\text { imagines the checklist could be used or would be used } \\
\text { in order to solve some barrier or to improve usage. } \\
\text { These comments can include changing the way that the } \\
\text { pain checklist is used (e.g., frequency, time, different } \\
\text { users), or adding something to the checklist. } \\
\text { Note: It may be difficult to distinguish between } \\
\text { Imagined uses (im) and Enhanced/Informed practice } \\
\text { (en). The main difference between them is that en refer } \\
\text { to experiences that actually happened from using the } \\
\text { checklist, whereas im are speculated (e.g., if I were } \\
\text { to....I would use it like this...) }\end{array}$ \\
\hline
\end{tabular}

\section{PREFERENCE (pref)}

These comments refer to the staff member's preference for either the original PACSLAC or the revised version. These comments may include reasons for why they prefer one over the other, e.g., because one is shorter, or more concise etc. It should be clear based on the comment that the staff member is making a comparison and/or stating a preference.

\section{OTHER (oth)}

If comments do not fit into any of the above categories, it goes here. 


\section{Appendix G}

\section{University of Regina}

OFFICE OF RESEARCH SERVICES

$M$ EMORANDUM

DATE: November 1, 2011

TO: $\quad$ Sarah Chan

Psychology Department

FROM: Dr. Bruce Plouffe

Chair, Research Ethics Board

Re: $\quad$ Evidence-Based Development and Initial Validation of the Pain Assessment Checklist for Seniors with Limited Ability to Communicate-II (PACSLAC-11) (File \# 20S1112)

Please be advised that the University of Regina Research Ethics Board has reviewed your proposal and found it to be:

$\square 1$.

APPROVED AS SUBMITTED. Only applicants with this designation have ethical approval to proceed with their research as described in their applications. For research lasting more than one year (Section 1F). ETHICAL APPROVAL MUST BE RENEWED BY SUBMITTING A BRIEF STATUS REPORT EVERY

TWELVE MONTHS. Approval will be revoked unless a satisfactory status report is received. Any substantive changes in methodology or instrumentation must also be approved prior to their implementation.

2. ACCEPTABLE SUBJECT TO MINOR CHANGES AND PRECAUTIONS (SEE ATTACHED). Changes must be submitted to the REB and approved prior to beginning research. Please submit a supplementary memo addressing the concerns to the Chair of the REB. ${ }^{* *}$ Do not submit a new application. Once changes are deemed acceptable, ethical approval will be granted.

3. ACCEPTABLE SUBJECT TO CHANGES AND PRECAUTIONS (SEE ATTACHED). Changes must be submitted to the REB and approved prior to beginning research. Please submit a supplementary memo addressing the concerns to the Chair of the REB. ${ }^{* *}$ Do not submit a new application. Once changes are deemed acceptable, ethical approval will be granted.

4. UNACCEPTABLE AS SUBMITTED. The proposal requires substantial additions or redesign. Please contact the Chair of the REB for advice on how the project proposal might be revised.

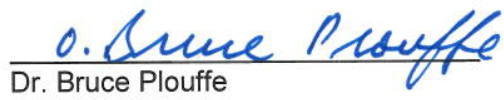

cc: Dr. Thomas Hadjistavropoulos - Psychology Department

** supplementary memo should be forwarded to the Chair of the Research Ethics Board at the Office of Research Services (Research and Innovation Centre, Room 109) or by e-mail to research.ethics@uregina.ca 